\title{
Pyridine-4(1H)-one Alkaloids from Waltheria indica as Antitrypanosomatid Agents
}

Sylvian Cretton, ${ }^{\dagger,}$ Marcel Kaiser, ${ }^{\S, \wedge}$ Soumana Karimou, ${ }^{\|}$Samad Nejad Ebrahimi, ${ }^{\nabla}$ Pascal

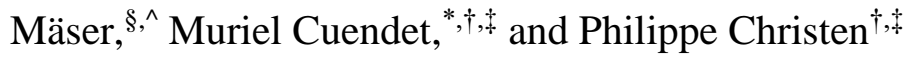

${ }^{\dagger}$ School of Pharmaceutical Sciences, University of Geneva, 1211 Geneva 4, Switzerland

* Institute of Pharmaceutical Sciences of Western Switzerland, University of Geneva, 1211

Geneva 4, Switzerland

${ }^{\S}$ Swiss Tropical and Public Health Institute, 4002 Basel, Switzerland

^University of Basel, 4003 Basel, Switzerland

\| Plantasav, B.P. 10308 Niamey, Niger

$\nabla$ Department of Phytochemistry, Medicinal Plants and Drugs Research Institute, Shahid Beheshti University, G. C., Evin, Tehran, Iran

*To whom correspondence should be addressed. Tel.: +41 2237933 86. Fax: +41 22 37933 99. E-mail:

Muriel.cuendet@unige.ch 


\section{$\underline{\text { List of Contents }}$}

No.

Figure S1 Chemical structure of waltheriones M to Q

Figure S2 UV spectrum of 8-Demethoxywaltherione F (1)

Figure S3 HRESIMS spectrum of 8-Demethoxywaltherione F (1)

Figure S4 $\quad{ }^{1} \mathrm{H}$ NMR spectrum of 8-Demethoxywaltherione F (1)

Figure S5 COSY spectrum of 8-Demethoxywaltherione F (1)

Figure S6 $\quad$ HSQC spectrum of 8-Demethoxywaltherione F (1)

Figure S7 HMBC spectrum of 8-Demethoxywaltherione F (1)

Figure S8 ROESY spectrum of 8-Demethoxywaltherione F (1)

Figure S9 UV spectrum of Waltherione R (2)

Figure S10 HRESIMS spectrum of Waltherione R (2)

Figure S11 $\quad{ }^{1} \mathrm{H}$ NMR spectrum of Waltherione R (2)

Figure S12 COSY spectrum of Waltherione R (2)

Figure S13 HSQC spectrum of Waltherione R (2)

Figure S14 HMBC spectrum of Waltherione R (2)

Figure S15 ROESY spectrum of Waltherione R (2)

Figure S16 UV spectrum of 1-Methoxywaltherione O (3)

Figure S17 HRESIMS spectrum of 1-Methoxywaltherione O (3)

Figure S18 $\quad{ }^{1} \mathrm{H}$ NMR spectrum of 1-Methoxywaltherione O (3)

Figure S19

Figure S20

Figure S21

Figure S22

Figure S23

Figure S24
COSY spectrum of 1-Methoxywaltherione $\mathrm{O}(\mathbf{3})$

HSQC spectrum of 1-Methoxywaltherione O (3)

HMBC spectrum of 1-Methoxywaltherione O (3)

ROESY spectrum of 1-Methoxywaltherione O (3)

UV spectrum of 15-Hydroxywaltherione G (4)

HRESIMS spectrum of 15-Hydroxywaltherione G (4)
Page

6

6

6

7

7

8

8 
Figure S25 $\quad{ }^{1} \mathrm{H}$ NMR spectrum of 15-Hydroxywaltherione G (4)

Figure S26 COSY spectrum of 15-Hydroxywaltherione G (4) 18

Figure S27 HSQC spectrum of 15-Hydroxywaltherione G (4) 18

Figure S28 HMBC spectrum of 15-Hydroxywaltherione G (4) 19

Figure S29 ROESY spectrum of 15-Hydroxywaltherione G (4) 19

Figure S30 UV spectrum of 8-Hydroxywaltherione M (5) 20

$\begin{array}{lll}\text { Figure S31 HRESIMS spectrum of 8-Hydroxywaltherione M (5) } & 20\end{array}$

Figure S32 $\quad{ }^{1} \mathrm{H}$ NMR spectrum of 8-Hydroxywaltherione M (5) 21

Figure S33 COSY spectrum of 8-Hydroxywaltherione M (5) 21

Figure S34 HSQC spectrum of 8-Hydroxywaltherione M (5) 22

Figure S35 HMBC spectrum of 8-Hydroxywaltherione M (5) 22

Figure S36 ROESY spectrum of 8-Hydroxywaltherione M (5) 23

$\begin{array}{lll}\text { Figure S37 UV spectrum of Waltherione S (6) } & 23\end{array}$

$\begin{array}{lll}\text { Figure S38 HRESIMS spectrum of Waltherione S (6) } & 24\end{array}$

Figure S39 $\quad{ }^{1} \mathrm{H}$ NMR spectrum of Waltherione S (6) 24

$\begin{array}{lll}\text { Figure S40 HSQC spectrum of Waltherione S (6) } & 25\end{array}$

Figure S41 HMBC spectrum of Waltherione S (6) 25

$\begin{array}{lll}\text { Figure S42 ROESY spectrum of Waltherione S (6) } & 26\end{array}$

Figure S43 UV spectrum of Waltherione T (7) 26

$\begin{array}{lll}\text { Figure S44 HRESIMS spectrum of Waltherione T (7) } & 27\end{array}$

$\begin{array}{lll}\text { Figure S45 } & { }^{1} \mathrm{H} \text { NMR spectrum of Waltherione T (7) } & 27\end{array}$

$\begin{array}{lll}\text { Figure S46 HSQC spectrum of Waltherione T (7) } & 28\end{array}$

$\begin{array}{lll}\text { Figure S47 HMBC spectrum of Waltherione T (7) } & 28\end{array}$

$\begin{array}{lll}\text { Figure S48 ROESY spectrum of Waltherione T (7) } & 29\end{array}$

Figure S49 UV spectrum of 2-Hydroxymethylwaltherione C (8) 29

Figure S50 HRESIMS spectrum of 2-Hydroxymethylwaltherione C (8) 30 
Figure S51 $\quad{ }^{1} \mathrm{H}$ NMR spectrum of 2-Hydroxymethylwaltherione C (8) 30

Figure S52 COSY spectrum of 2-Hydroxymethylwaltherione C (8) 31

Figure S53 HSQC spectrum of 2-Hydroxymethylwaltherione C (8) 31

Figure S54 HMBC spectrum of 2-Hydroxymethylwaltherione C (8) 32

Figure S55 ROESY spectrum of 2-Hydroxymethylwaltherione C (8) 32

Figure S56 UV spectrum of 10-Hydroxywaltherione C (9) 33

Figure S57 HRESIMS spectrum of 10-Hydroxywaltherione C (9) 33

Figure S58 $\quad{ }^{1} \mathrm{H}$ NMR spectrum of 10-Hydroxywaltherione C (9) 34

Figure S59 COSY spectrum of 10-Hydroxywaltherione C (9) 34

Figure S60 HSQC spectrum of 10-Hydroxywaltherione C (9) 35

Figure S61 HMBC spectrum of 10-Hydroxywaltherione C (9) 35

Figure S62 ROESY spectrum of 10-Hydroxywaltherione C (9) 36

Figure S63 UV spectrum of Waltherione U (10) 36

$\begin{array}{lll}\text { Figure S64 HRESIMS spectrum of Waltherione U (10) } & 37\end{array}$

Figure S65 $\quad{ }^{1} \mathrm{H}$ NMR spectrum of Waltherione U (10) 37

Figure S66 COSY spectrum of Waltherione U (10) 38

Figure S67 HSQC spectrum of Waltherione U (10) 38

Figure S68 HMBC spectrum of Waltherione U (10) 39

$\begin{array}{lll}\text { Figure S69 } & \text { ROESY spectrum of Waltherione U (10) } & 39\end{array}$

$\begin{array}{lll}\text { Figure S70 UV spectrum of Waltherione V (11) } & 40\end{array}$

Figure S71 HRESIMS spectrum of Waltherione V (11) 40

Figure S72 $\quad{ }^{1} \mathrm{H}$ NMR spectrum of Waltherione V (11) 41

Figure S73 COSY spectrum of Waltherione V (11) 41

Figure S74 DEPTQ spectrum of Waltherione V (11) 42

Figure S75 HSQC spectrum of Waltherione V (11) 42

Figure S76 HMBC spectrum of Waltherione V (11) 43 
Figure S77 ROESY spectrum of Waltherione V (11) 43

Figure S78 UV spectrum of 13-Methoxywaltherione V (12) 44

Figure S79 HRESIMS spectrum of 13-Methoxywaltherione V (12) 44

Figure S80 $\quad{ }^{1} \mathrm{H}$ NMR spectrum of 13-Methoxywaltherione V (12) 45

Figure S81 COSY spectrum of 13-Methoxywaltherione V (12) 45

Figure S82 DEPTQ spectrum of 13-Methoxywaltherione V (12) 46

Figure S83 HSQC spectrum of 13-Methoxywaltherione V (12) 46

Figure S84 HMBC spectrum of 13-Methoxywaltherione V (12) 47

Figure S85 ROESY spectrum of 13-Methoxywaltherione V (12) 47

Figure S86 Calculation data for ECD curves of compounds 4, 8, 9, 11 and 12 


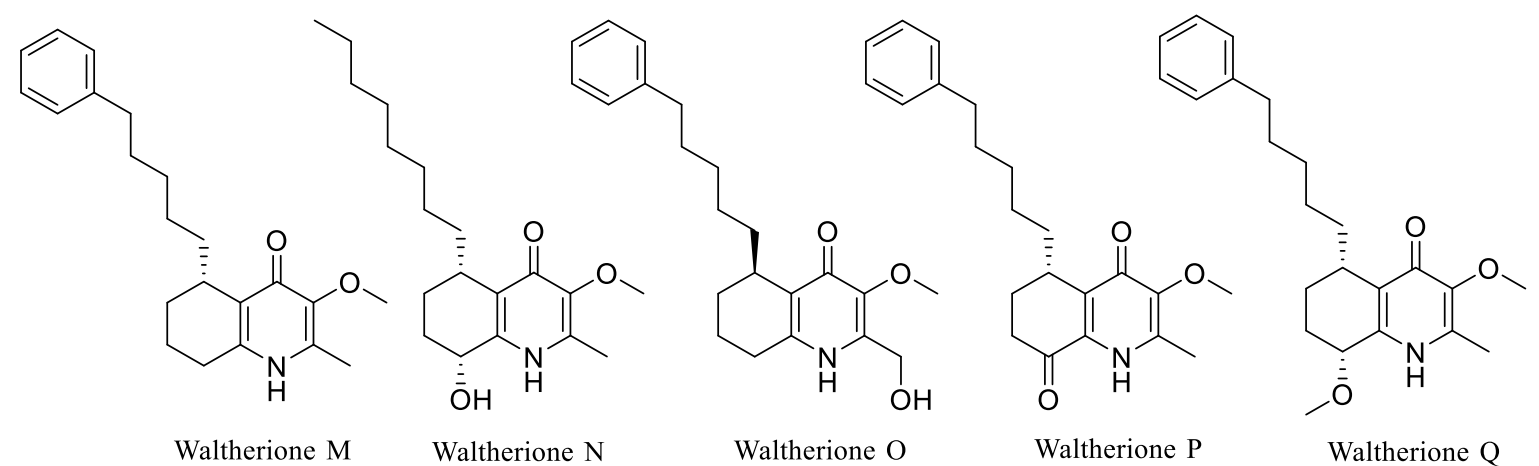

Figure S1. Chemical structure of waltheriones M to Q.

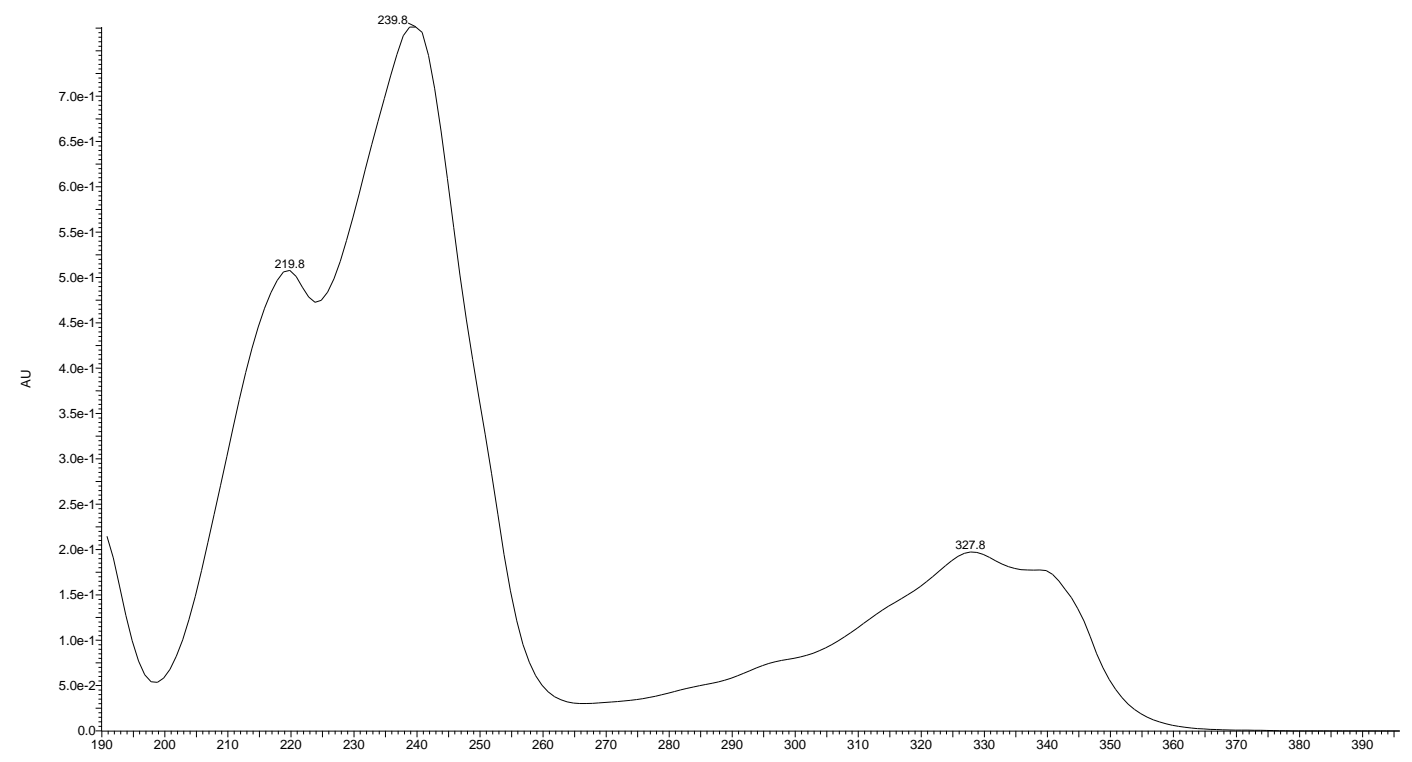

Figure S2. UV spectrum of 8-Demethoxywaltherione F (1).
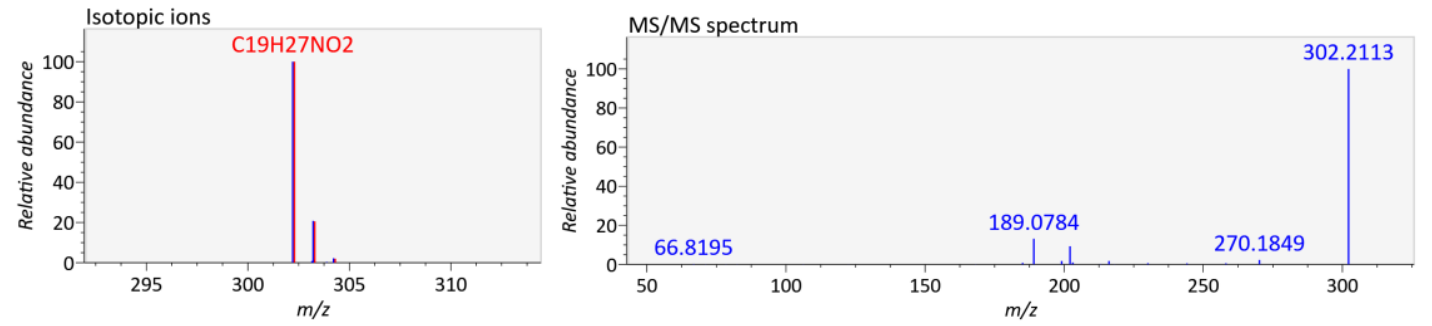

Figure S3. HRESIMS spectrum and MS/MS fragmentation of 8-Demethoxywaltherione F (1) (Q Exactive Focus Hybrid quadripole-orbitrap mass spectrometer, positive mode). 


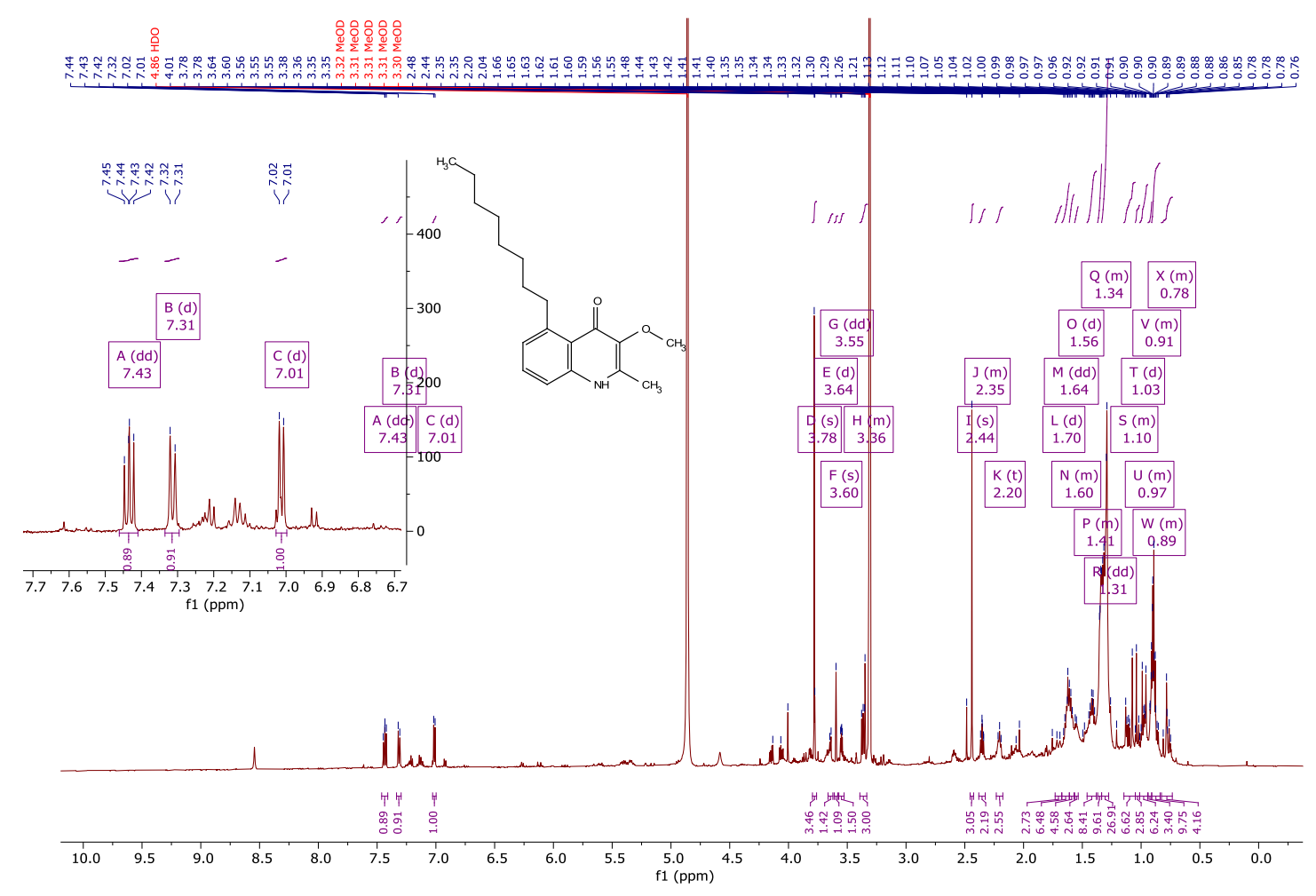

Figure S4. ${ }^{1} \mathrm{H}$ NMR (600 MHz, methanol- $\left.d_{4}\right)$ spectrum of 8-Demethoxywaltherione F (1).

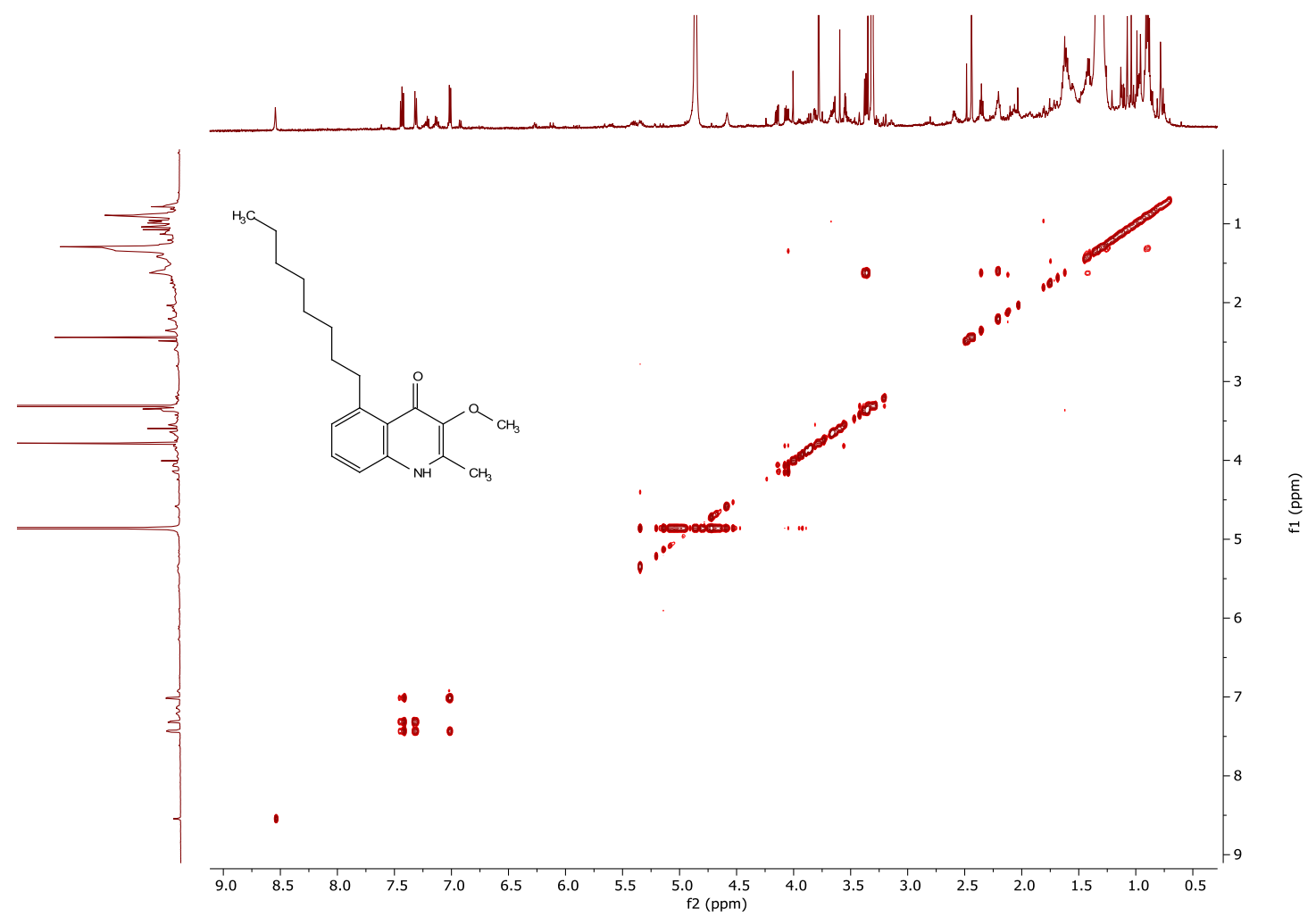

Figure S5. COSY (600 MHz, methanol- $d_{4}$ ) spectrum of 8-Demethoxywaltherione F (1). 


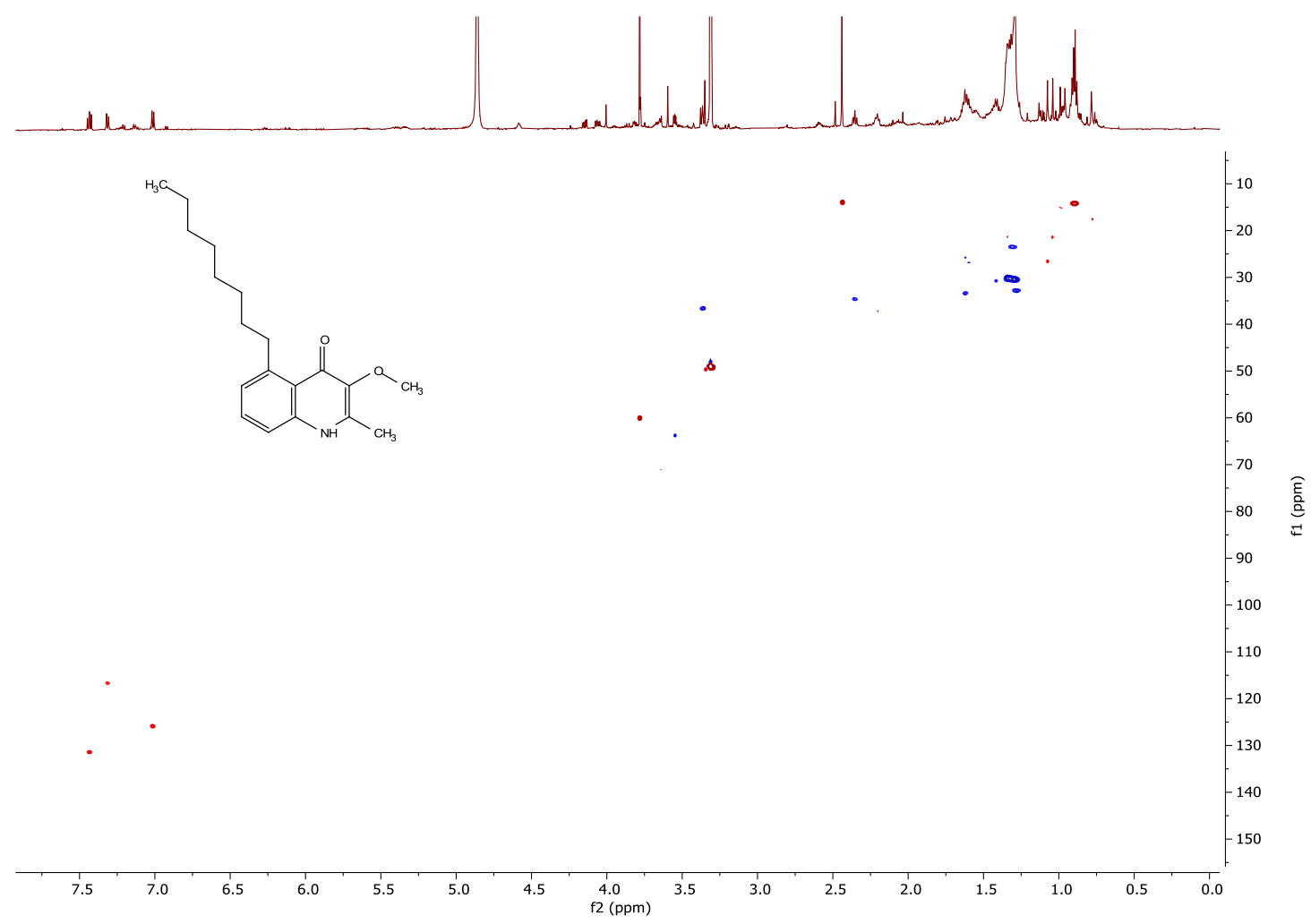

Figure S6. HSQC (600 MHz, methanol- $d_{4}$ ) spectrum of 8-Demethoxywaltherione F (1).

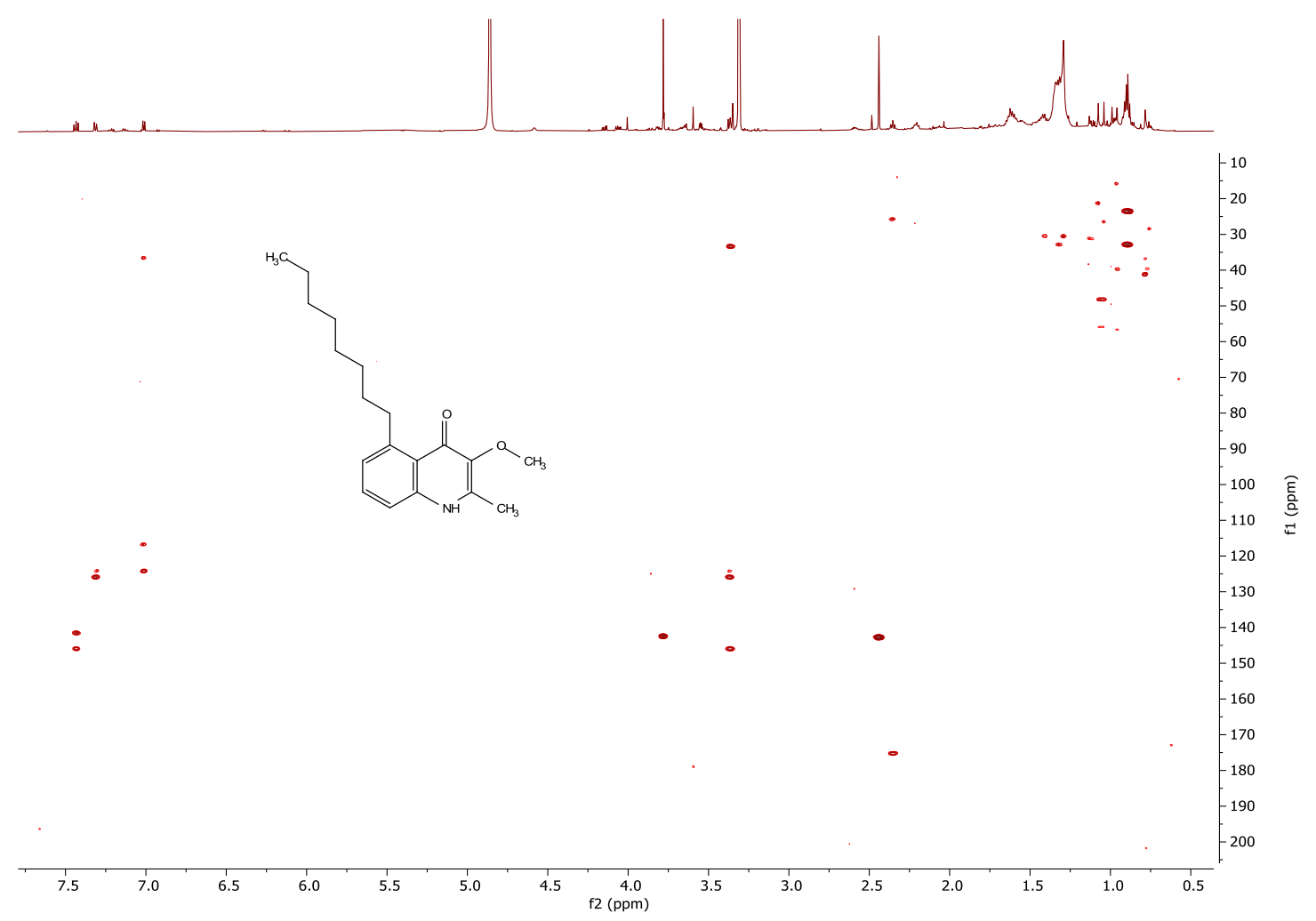

Figure S7. HMBC (600 MHz, methanol- $d_{4}$ ) spectrum of 8-Demethoxywaltherione F (1). 


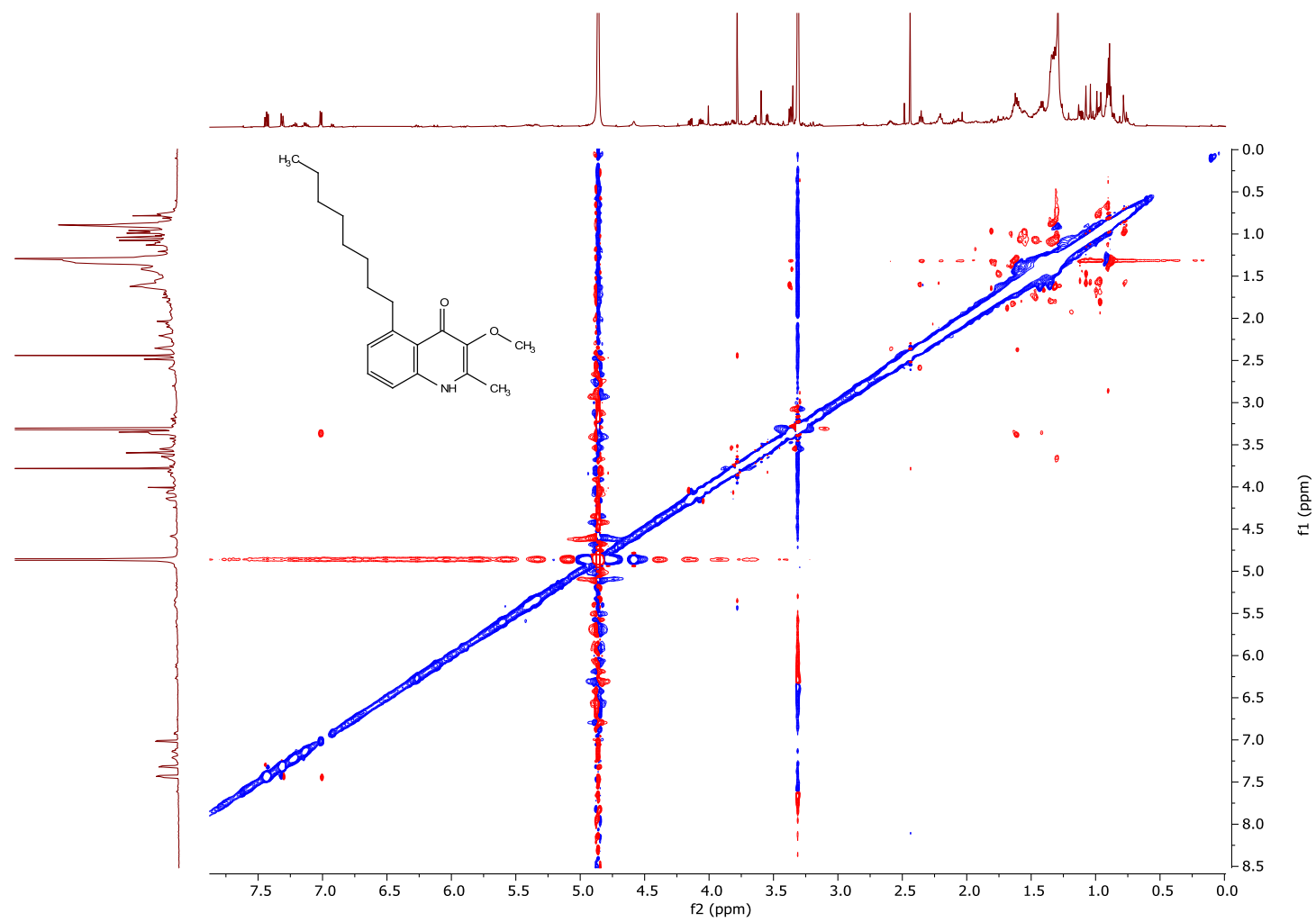

Figure S8. ROESY (600 MHz, methanol- $d_{4}$ ) spectrum of 8-Demethoxywaltherione F (1).

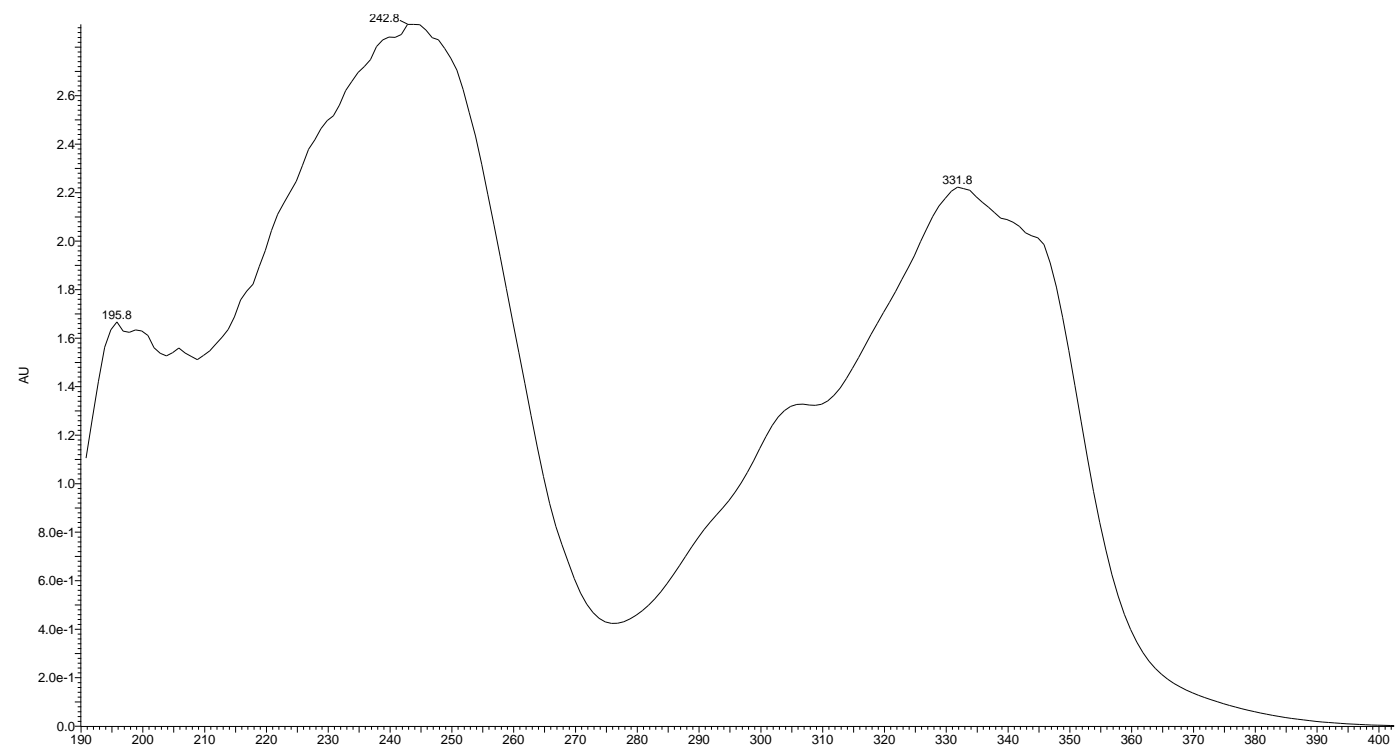

Figure S9. UV spectrum of Waltherione R (2). 

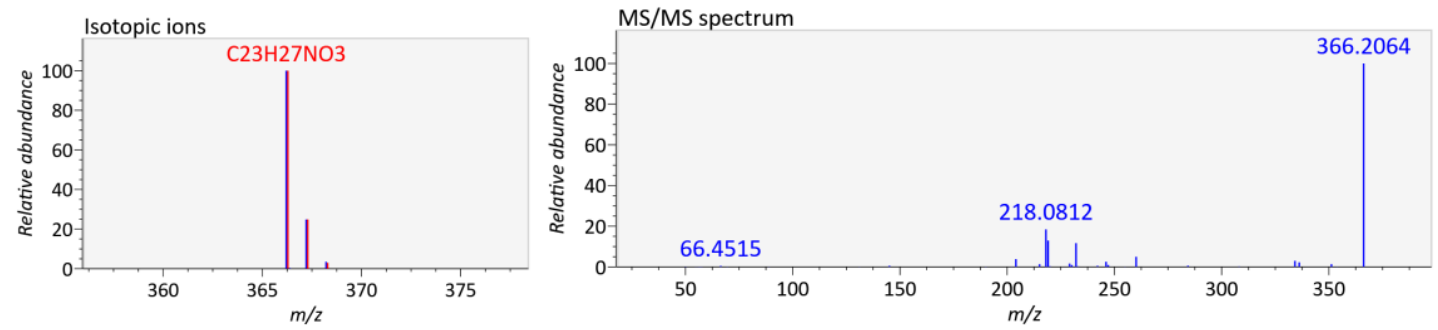

Figure S10. HRESIMS spectrum and MS/MS fragmentation of Waltherione R (2) (Q Exactive Focus Hybrid quadripole-orbitrap mass spectrometer, positive mode).

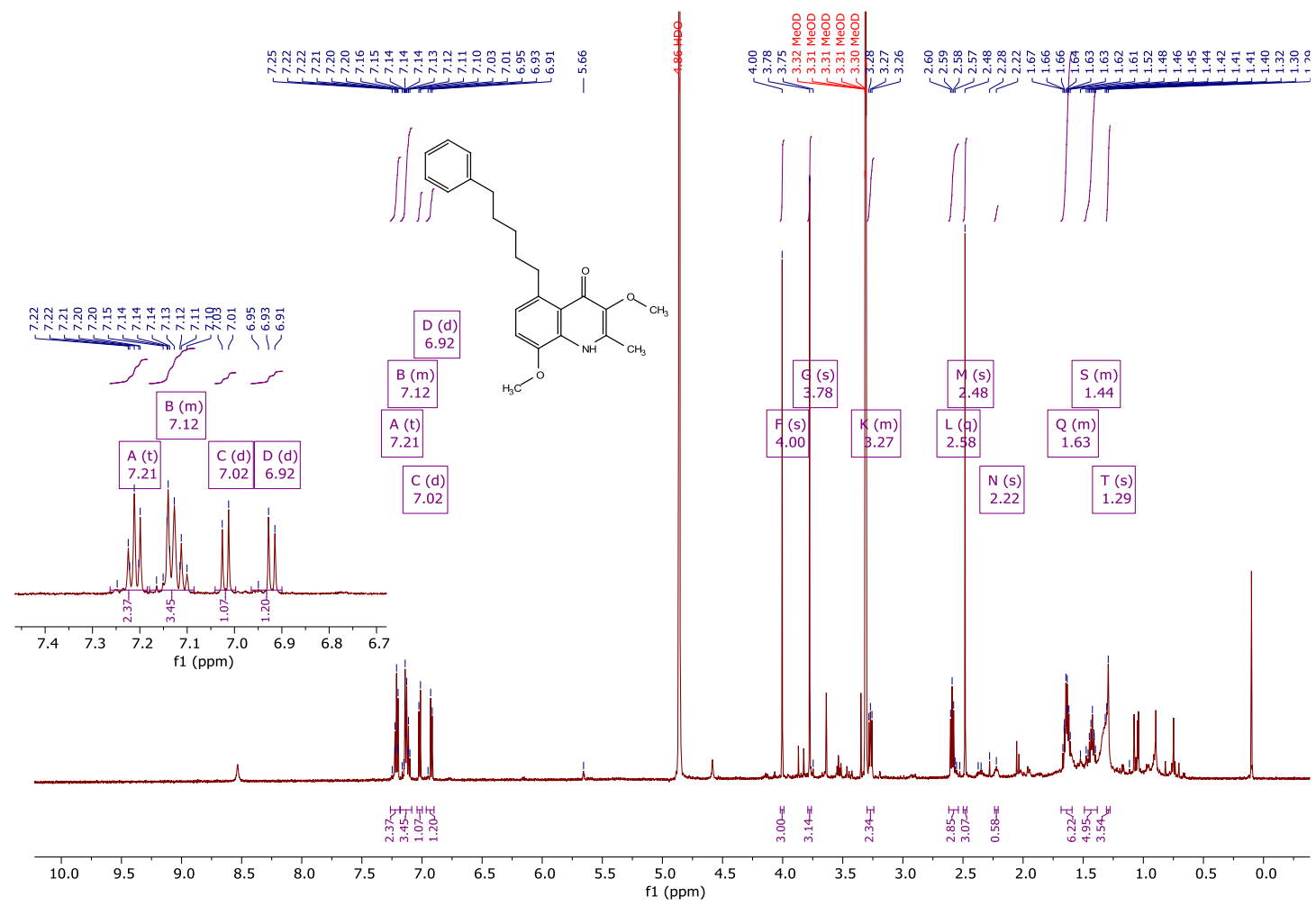

Figure S11. ${ }^{1} \mathrm{H}$ NMR (600 MHz, methanol- $\left.d_{4}\right)$ spectrum of Waltherione R (2). 


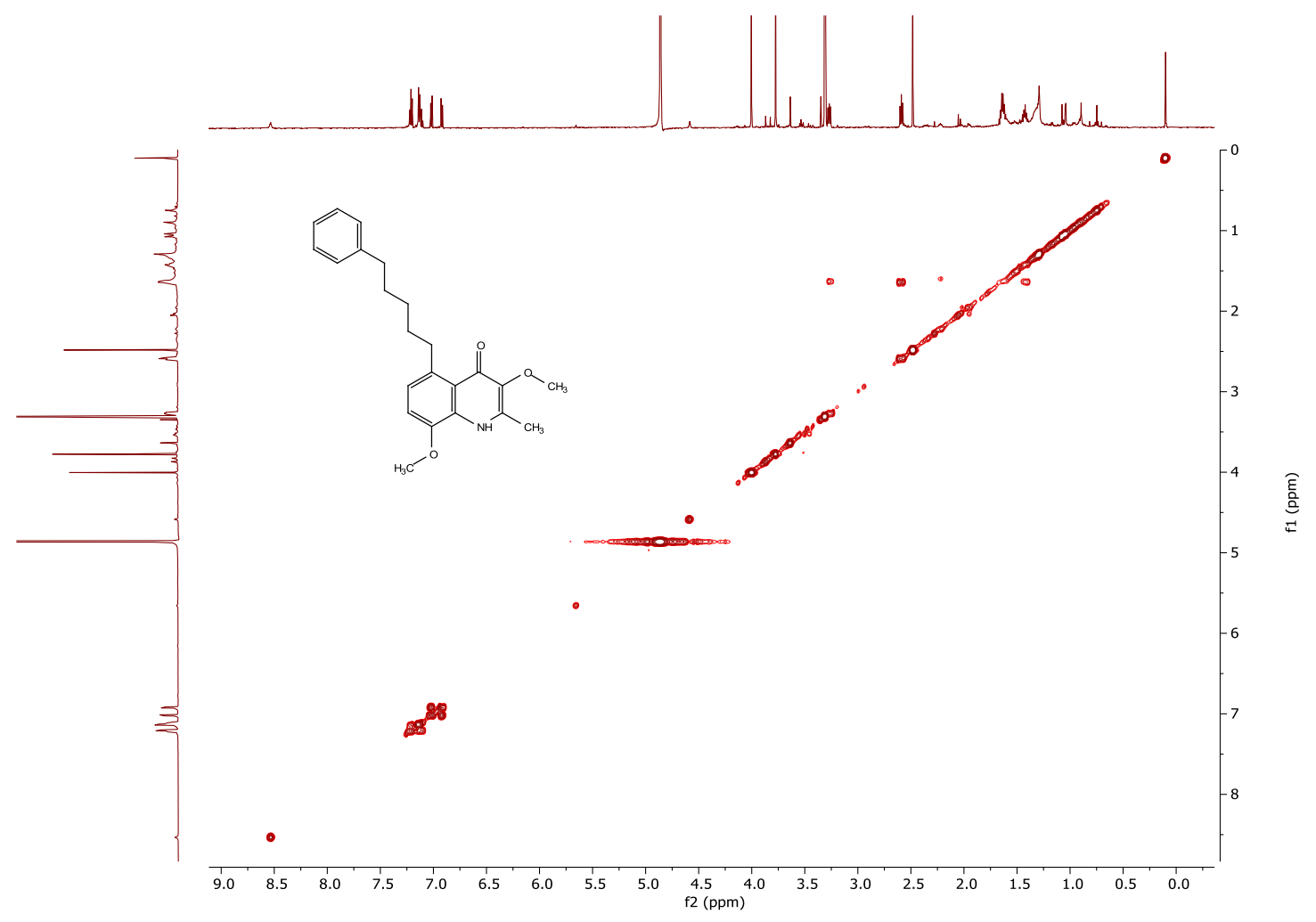

Figure S12. COSY (600 MHz, DMSO- $d_{6}$ ) spectrum of Waltherione R (2).

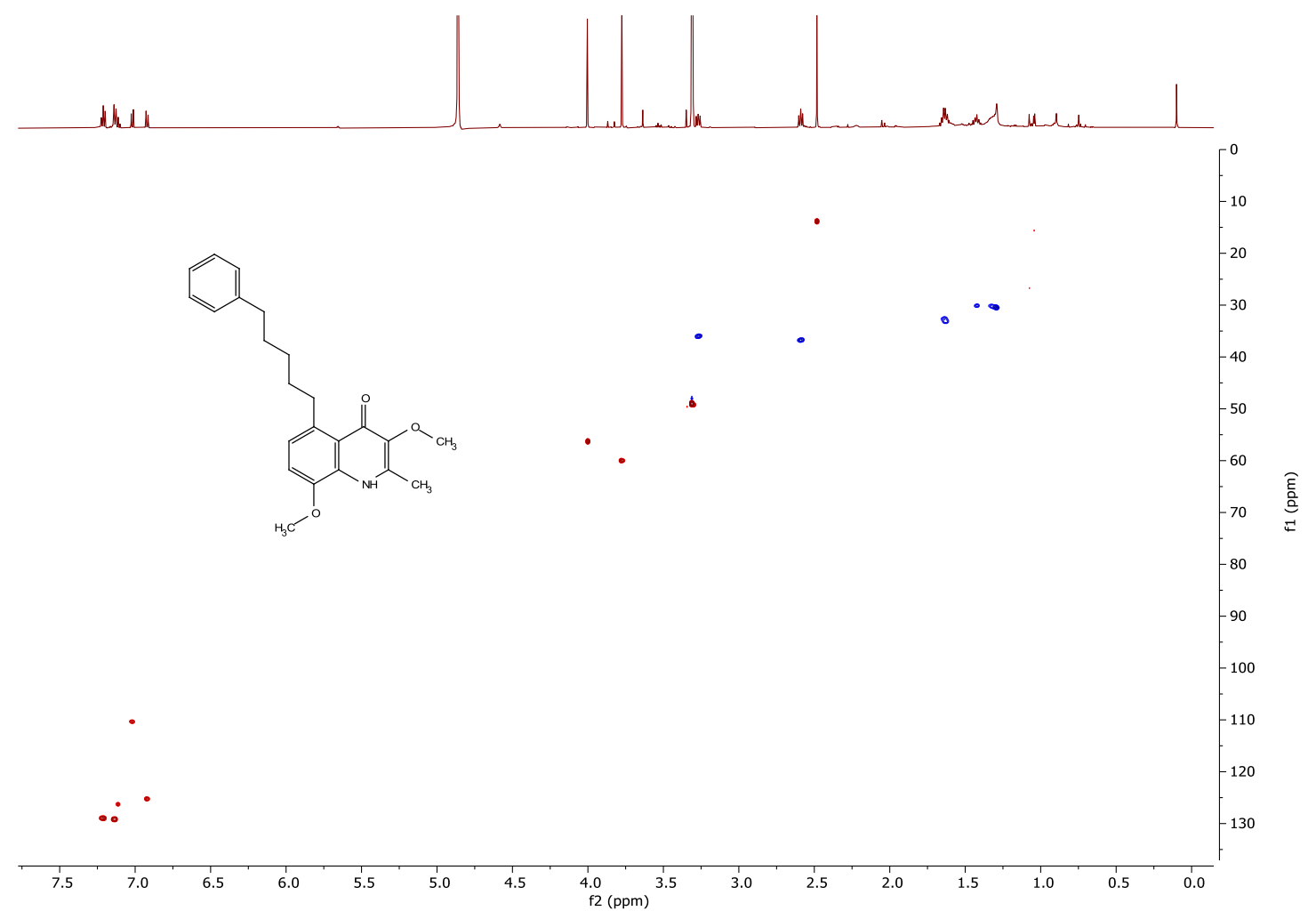

Figure S13. HSQC (600 MHz, methanol- $\left.d_{4}\right)$ spectrum of Waltherione R (2). 


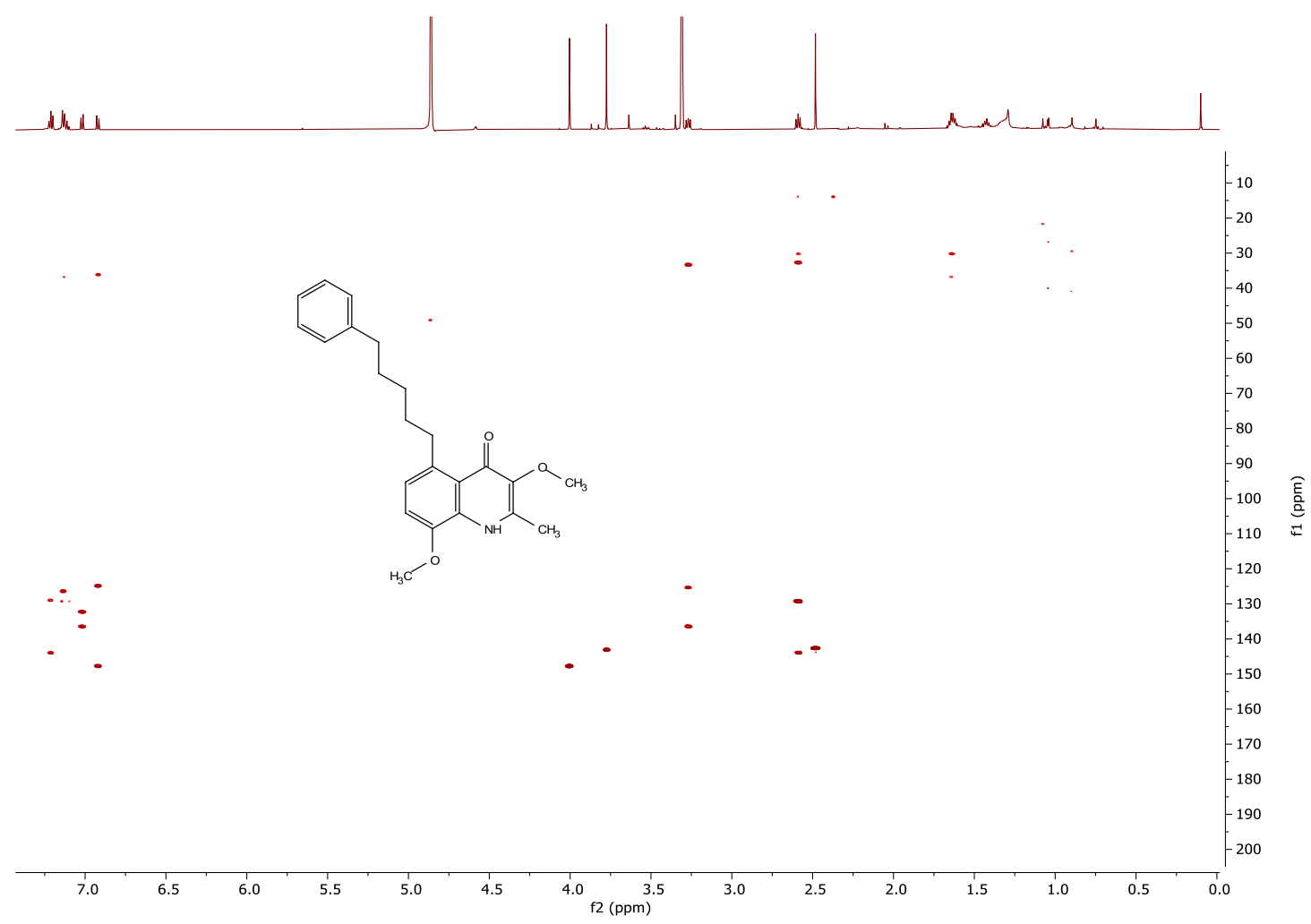

Figure S14. HMBC (600 MHz, methanol- $d_{4}$ ) spectrum of Waltherione R (2).

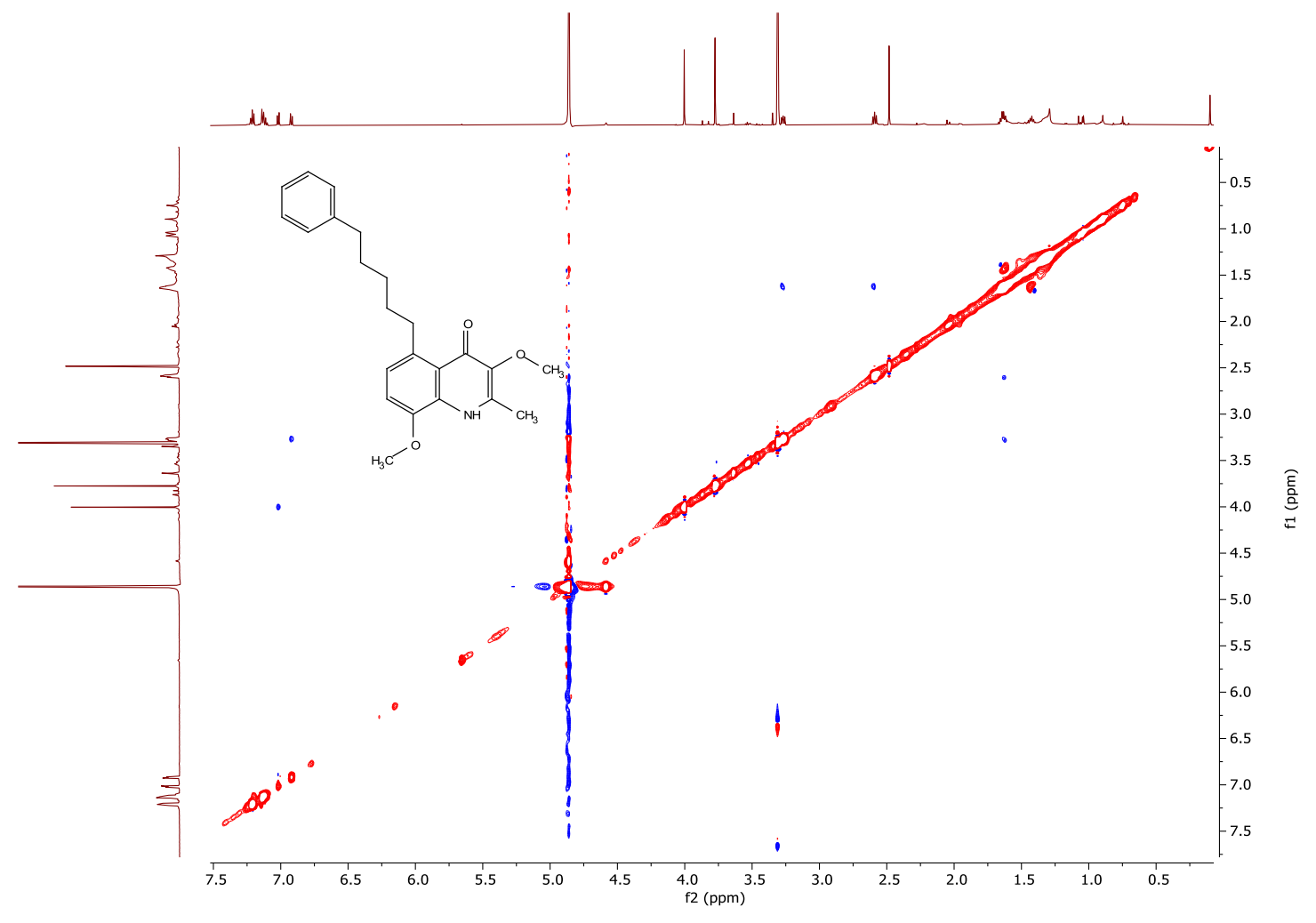

Figure S15. ROESY (600 MHz, methanol- $d_{4}$ ) spectrum of Waltherione R (2). 


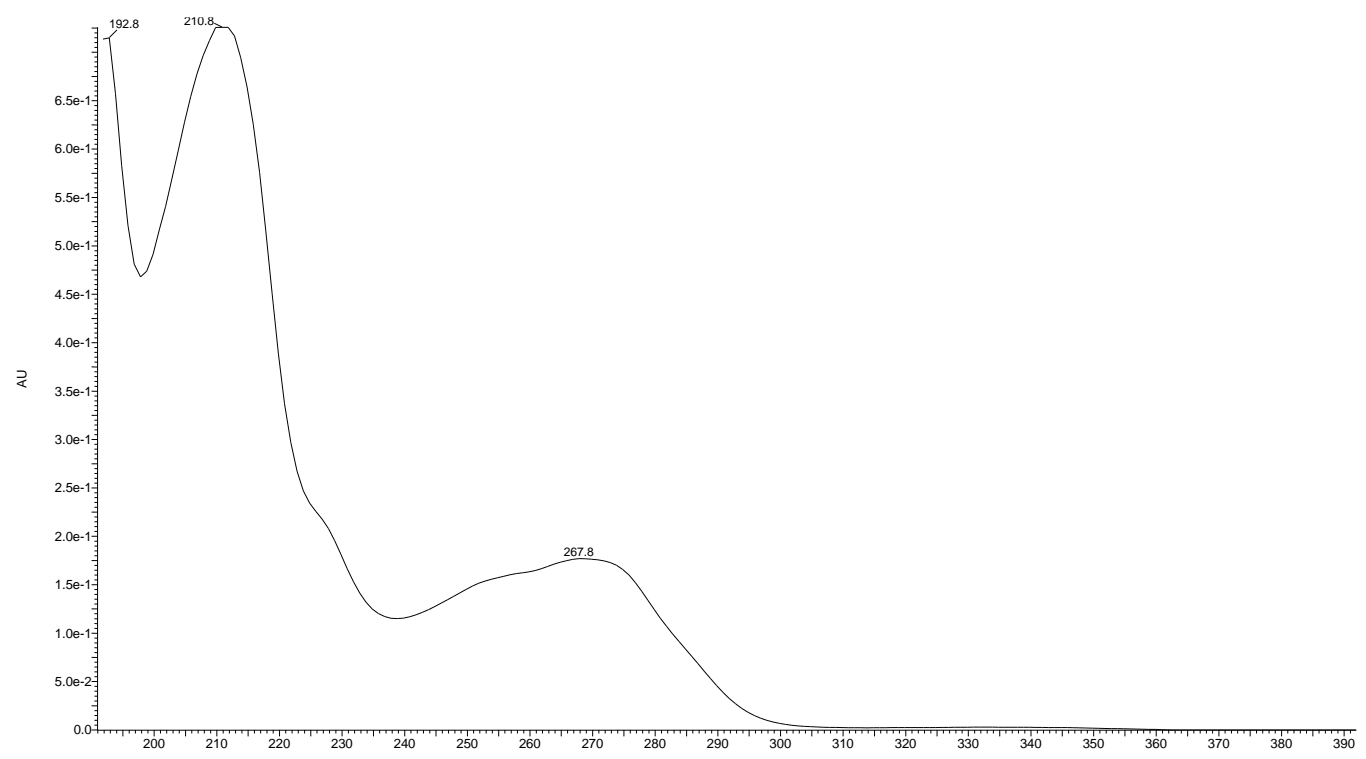

Figure S16. UV spectrum of 1-Methoxywaltherione O (3).
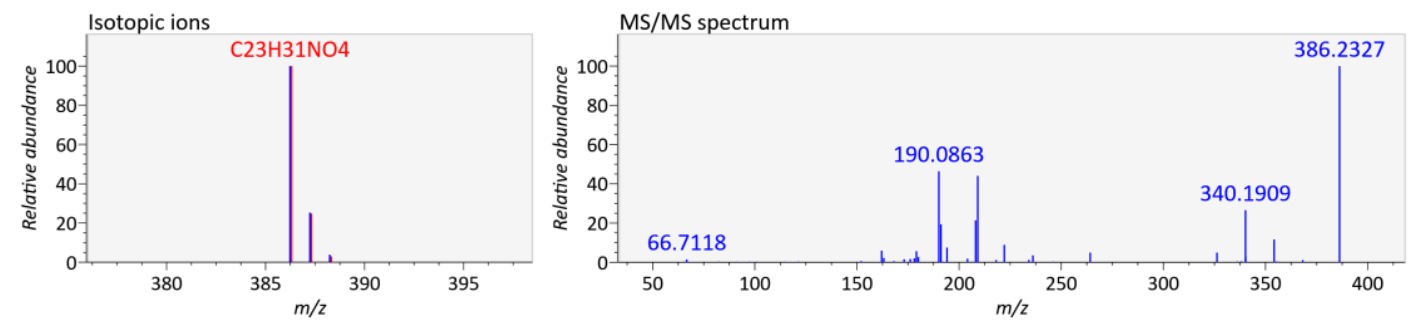

Figure S17. HRESIMS spectrum and MS/MS fragmentation of 1-Methoxywaltherione O (3) (Q Exactive Focus Hybrid quadripole-orbitrap mass spectrometer, positive mode). 


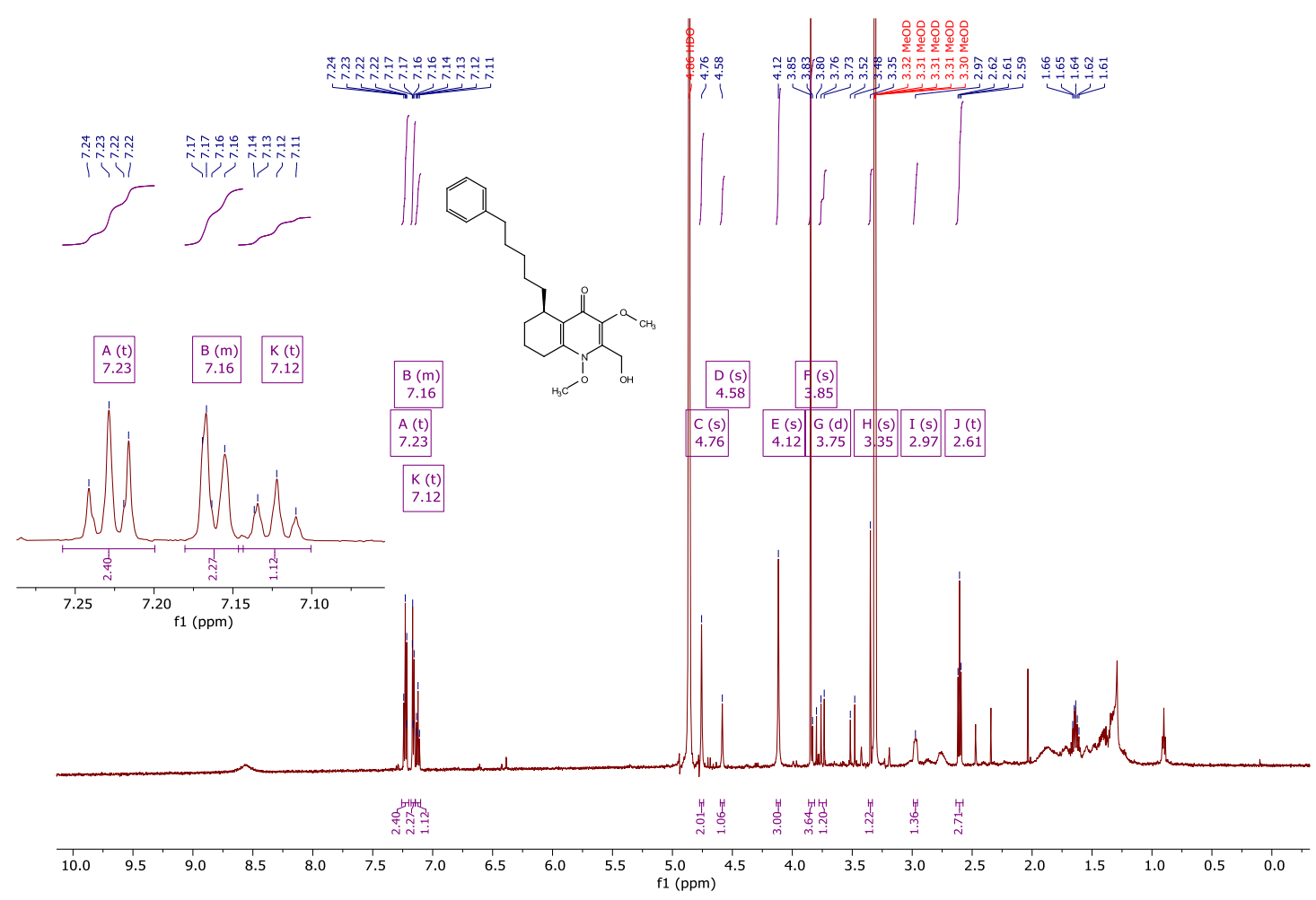

Figure S18. ${ }^{1} \mathrm{H}$ NMR $\left(600 \mathrm{MHz}\right.$, methanol- $\left.d_{4}\right)$ spectrum of 1-Methoxywaltherione $\mathrm{O}(3)$.

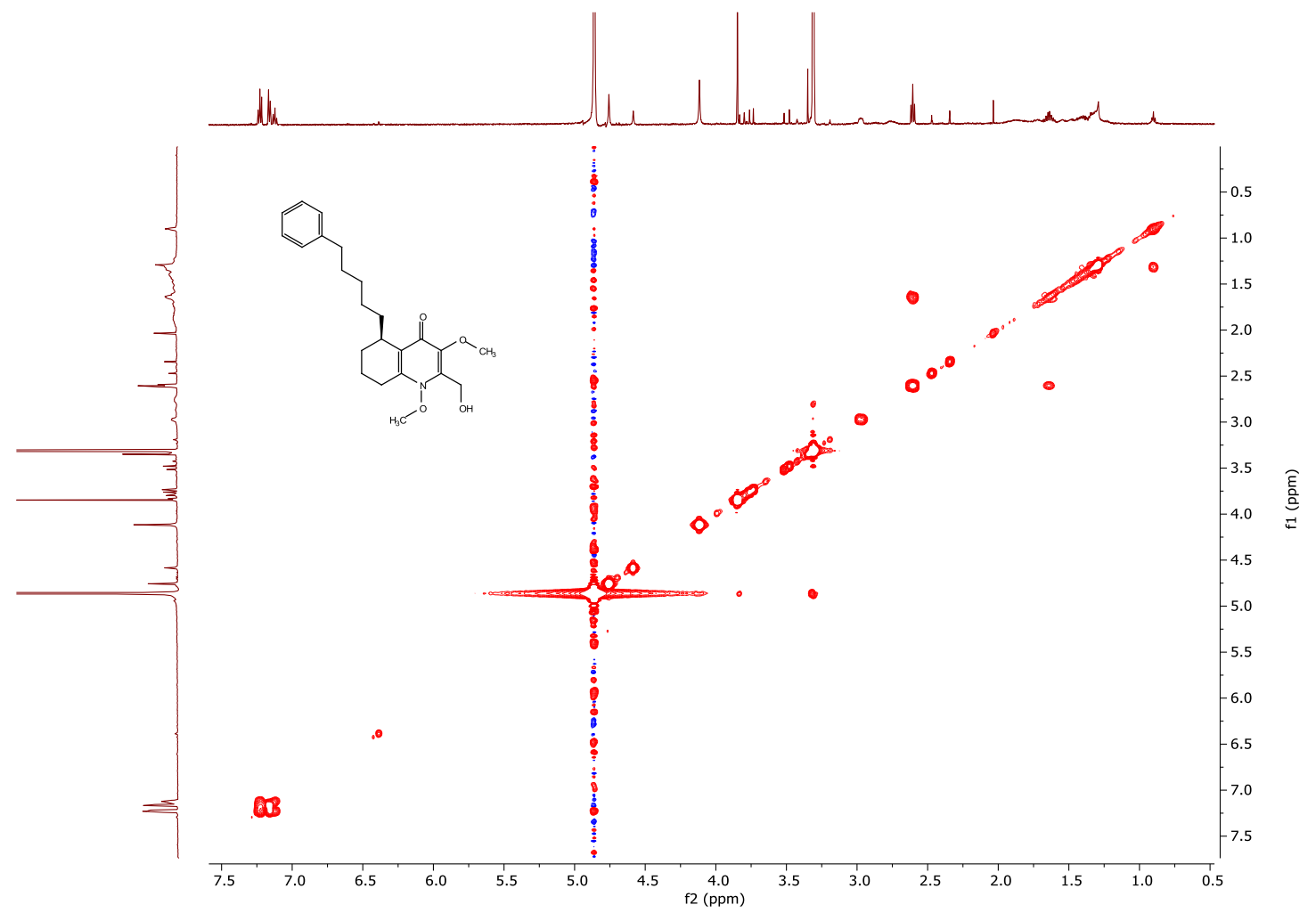

Figure S19. COSY (600 MHz, methanol- $\left.d_{4}\right)$ spectrum of 1-Methoxywaltherione O (3). 


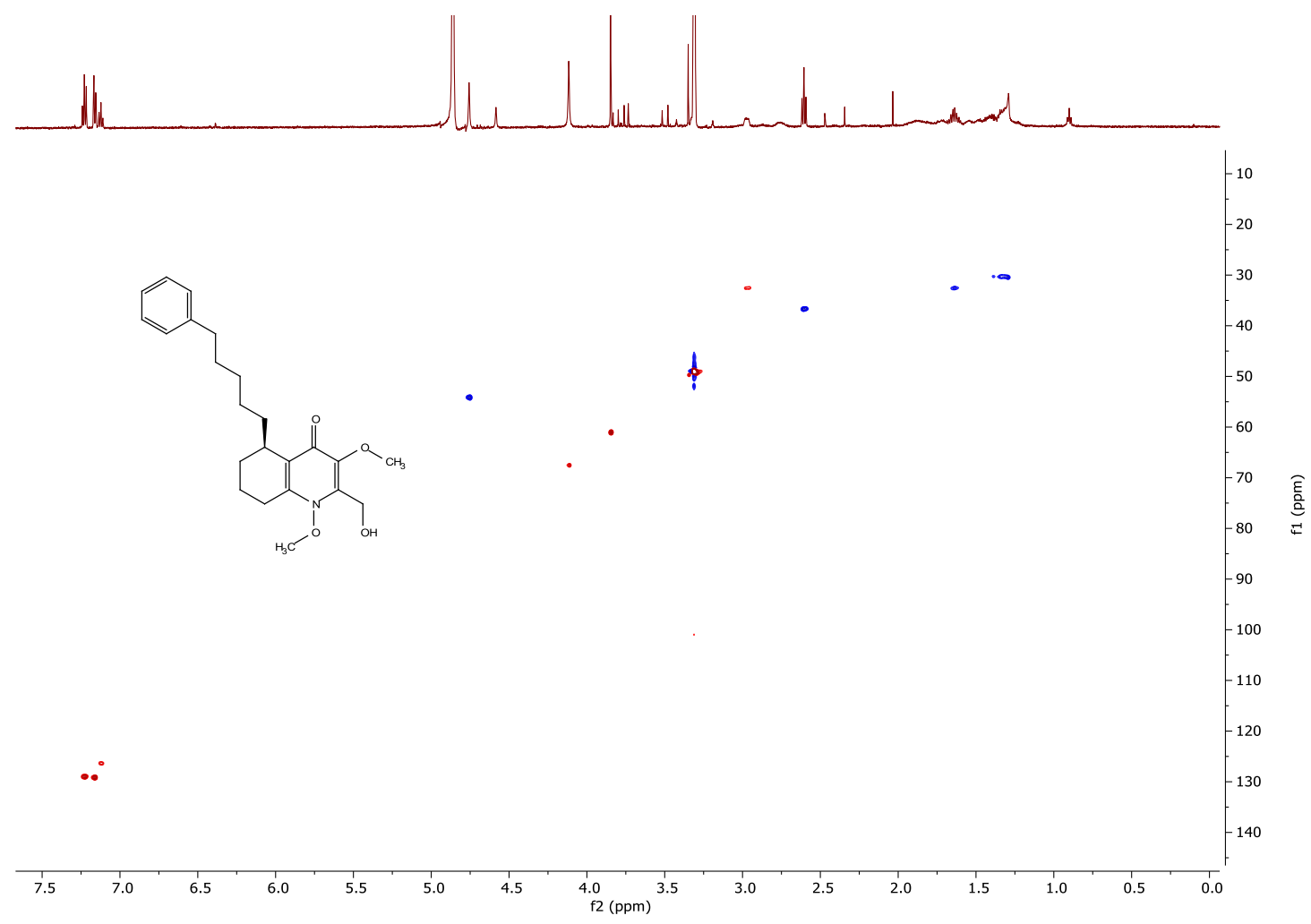

Figure S20. HSQC (600 MHz, methanol- $\left.d_{4}\right)$ spectrum of 1-Methoxywaltherione O (3).

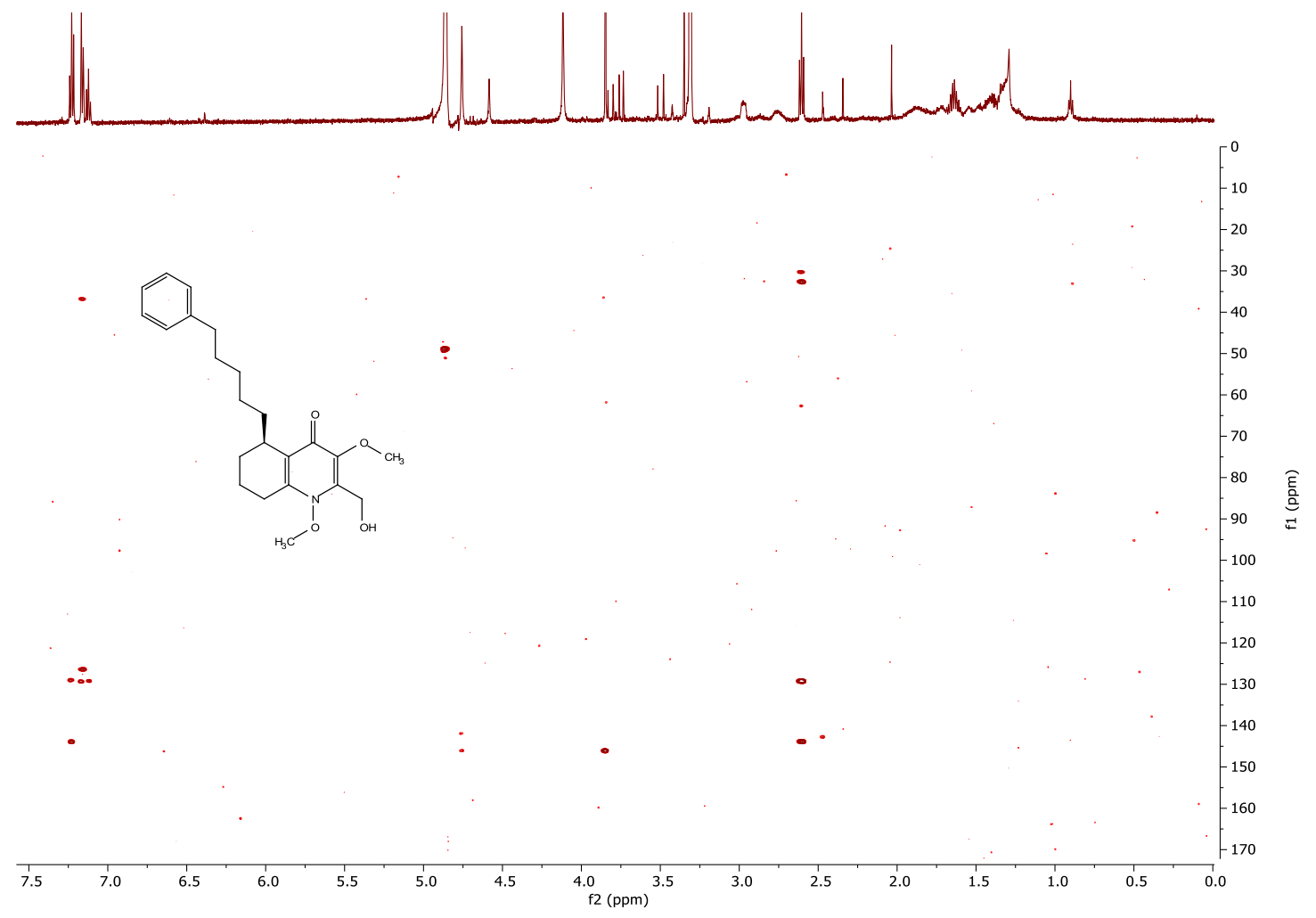


Figure S21. HMBC (600 MHz, methanol- $\left.d_{4}\right)$ spectrum of 1-Methoxywaltherione O (3).

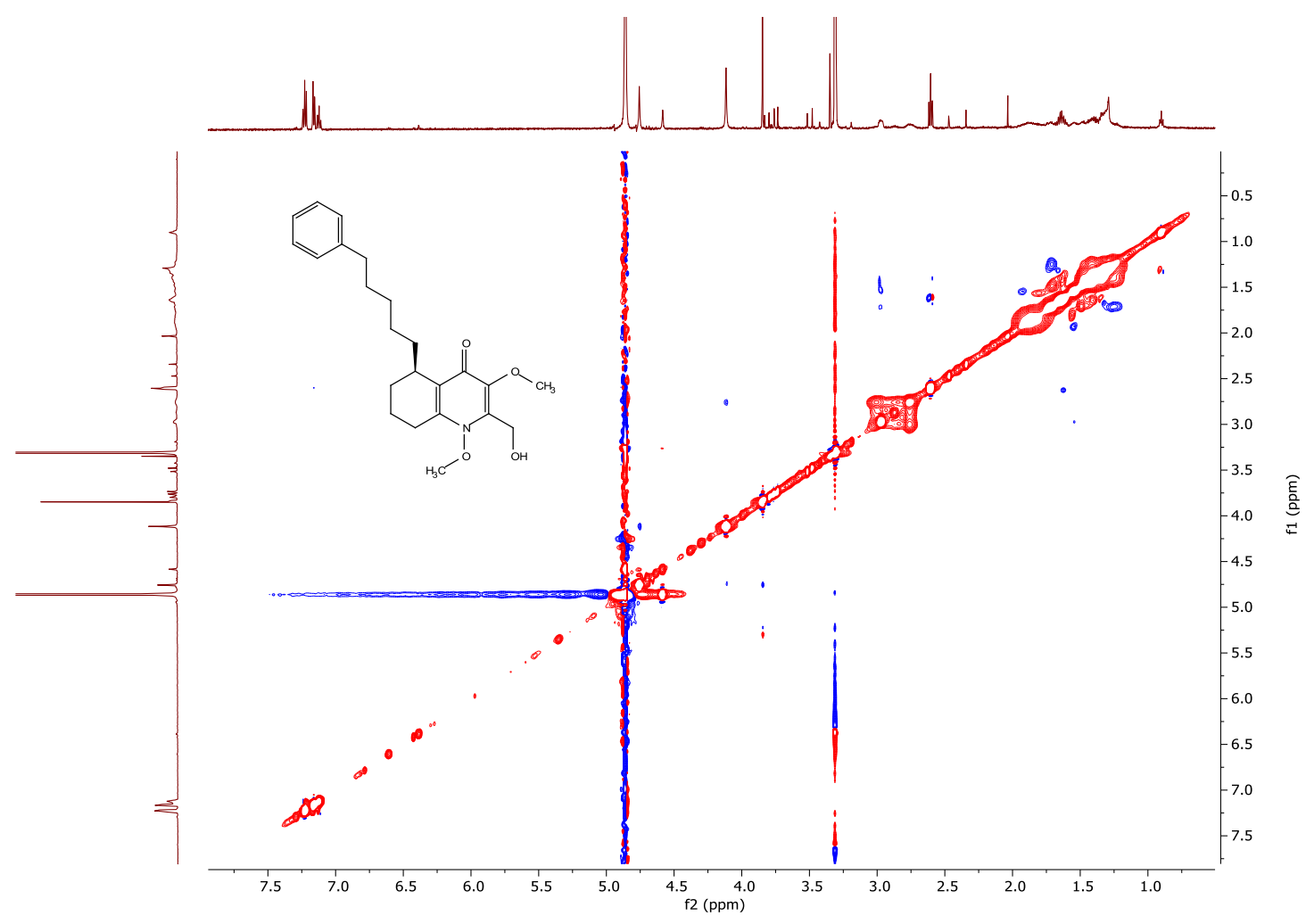

Figure S22. ROESY (600 MHz, methanol- $\left.d_{4}\right)$ spectrum of 1-Methoxywaltherione O (3).

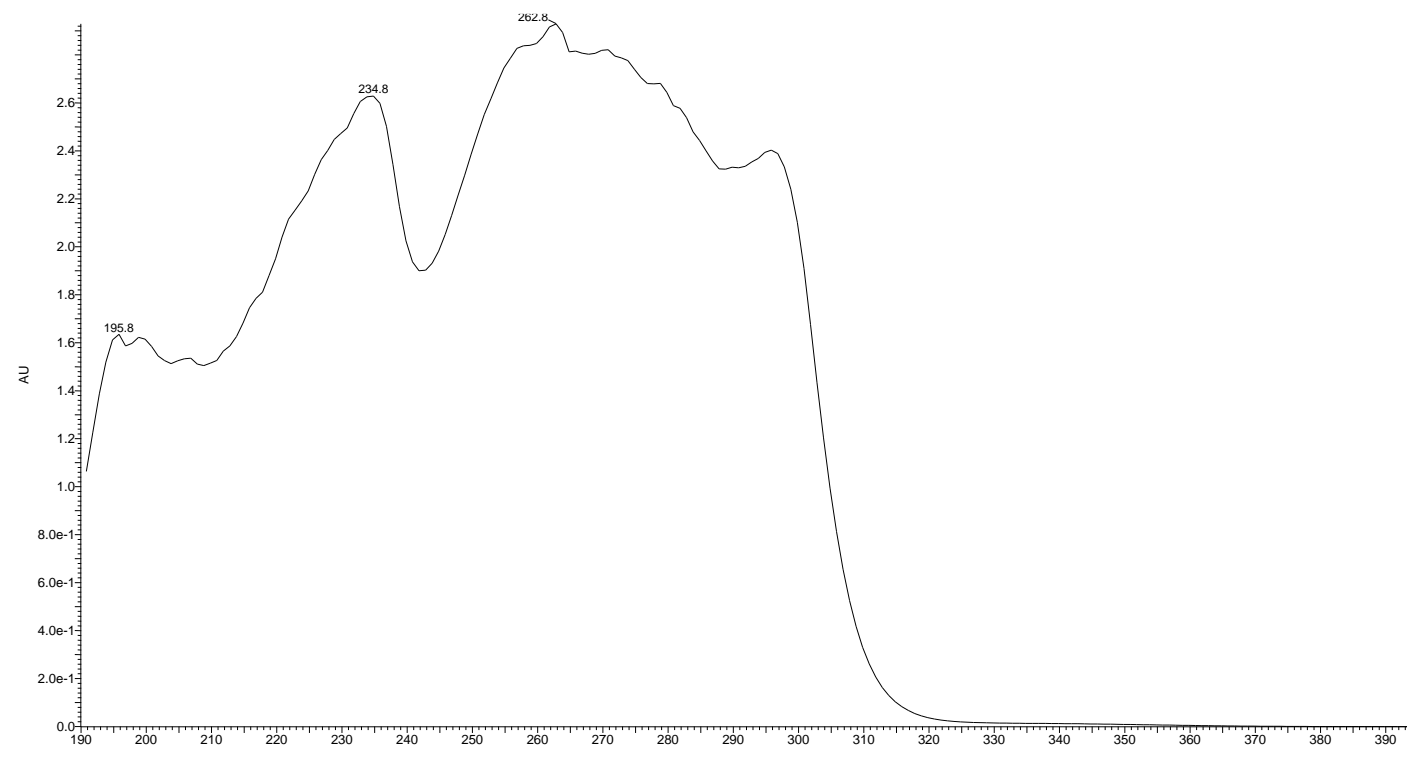

Figure S23. UV spectrum of 15-Hydroxywaltherione G (4). 

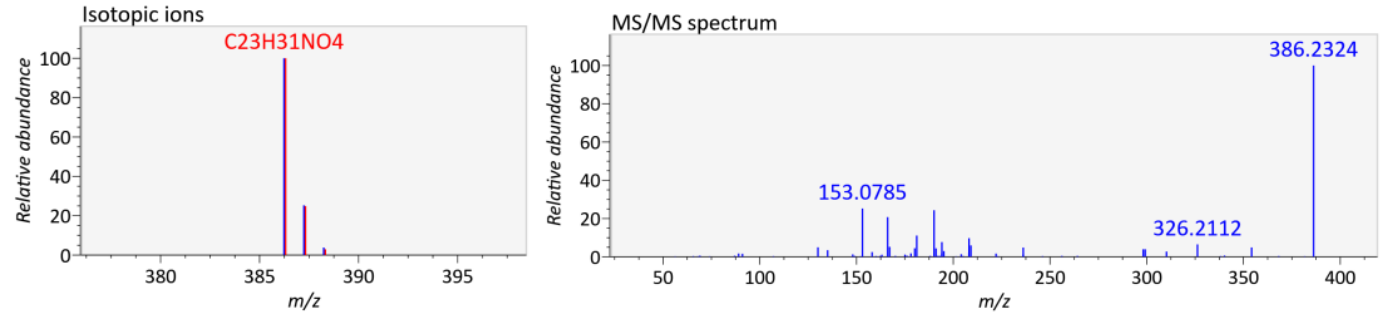

Figure S24. HRESIMS spectrum and MS/MS fragmentation of 15-Hydroxywaltherione G (4) (Q Exactive Focus Hybrid quadripole-orbitrap mass spectrometer, positive mode).

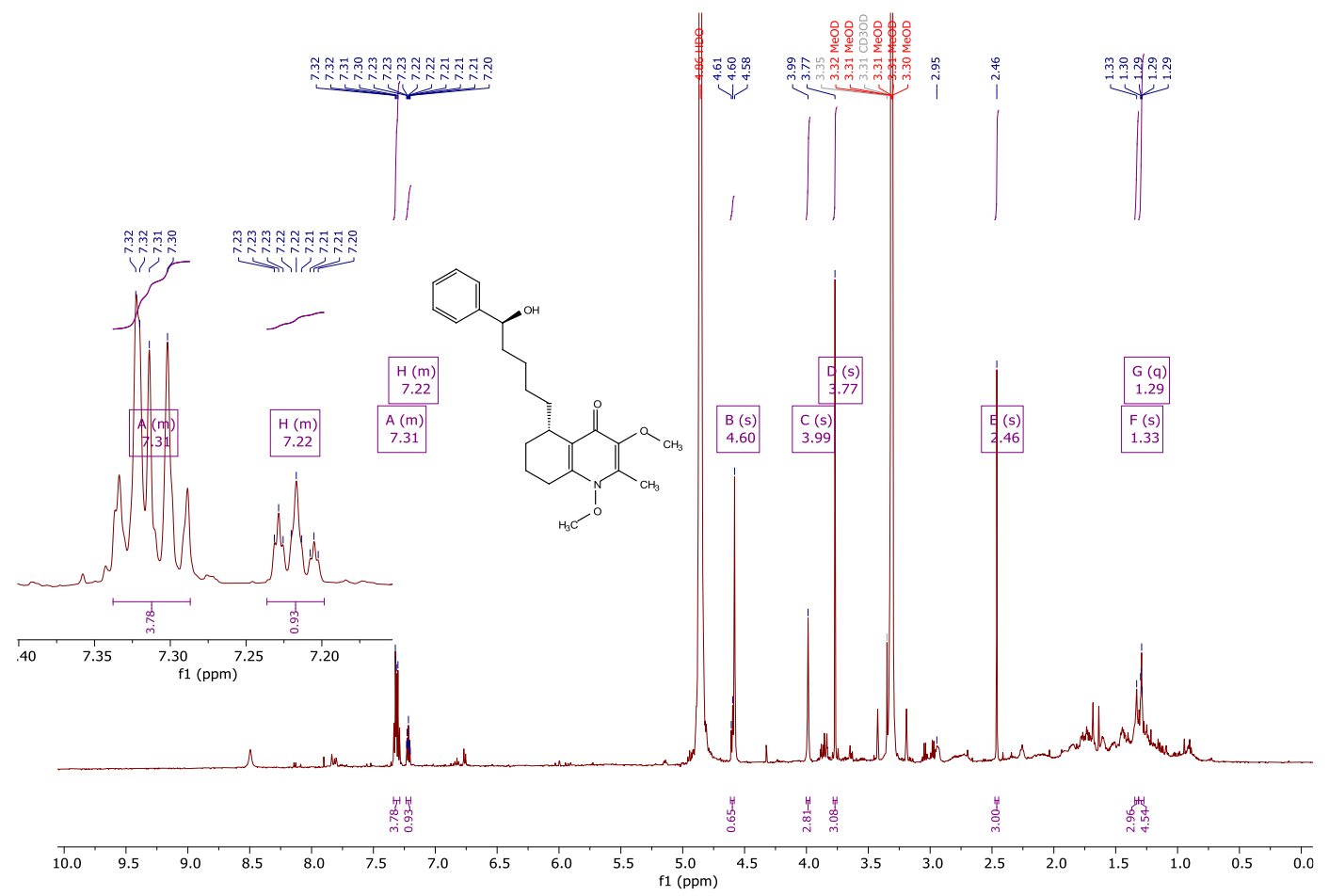

Figure S25. ${ }^{1} \mathrm{H}$ NMR (600 MHz, methanol- $\left.d_{4}\right)$ spectrum of 15-Hydroxywaltherione G (4). 


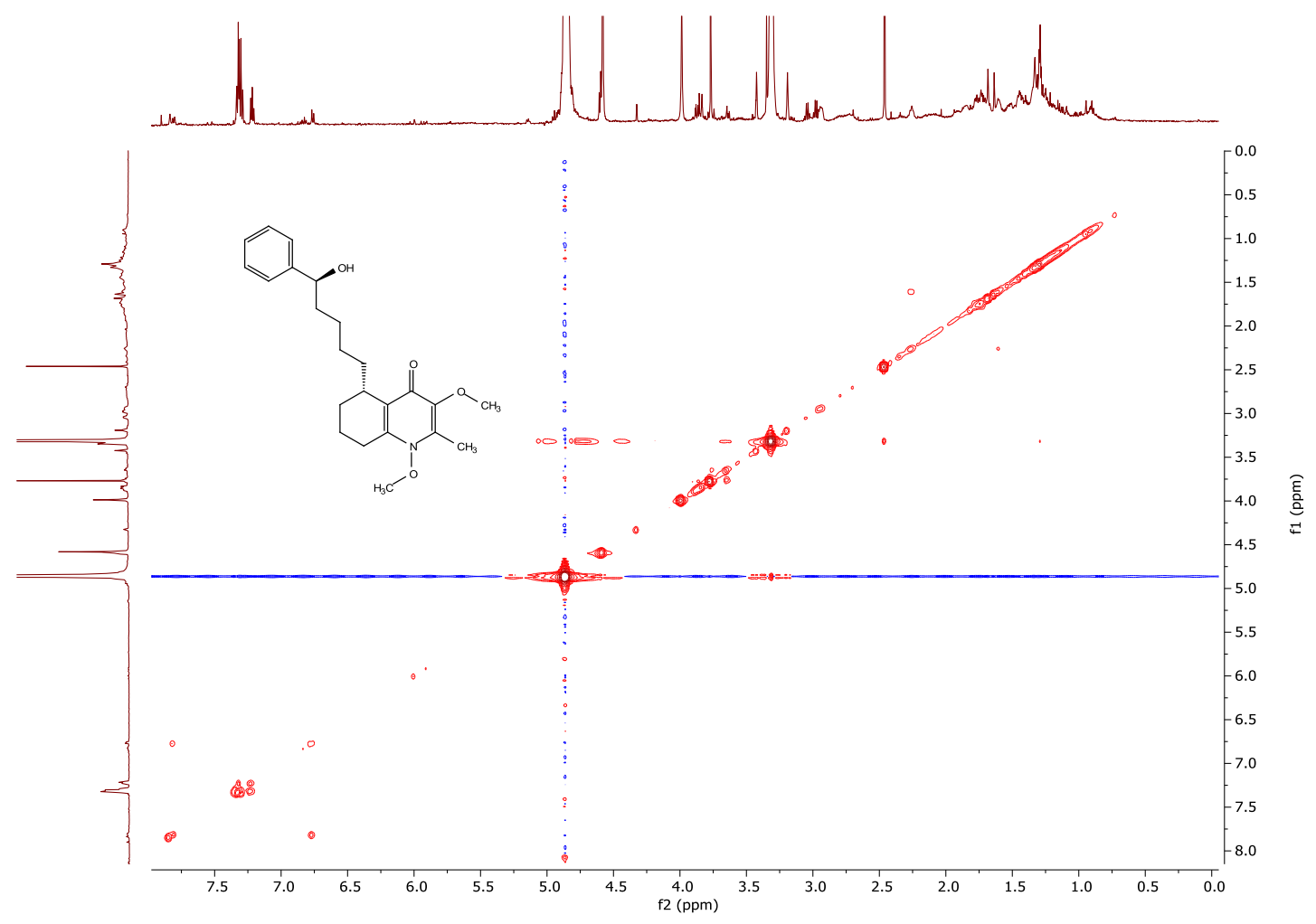

COSY (600 MHz, methanol-d4) spectrum of 15-Hydroxywaltherione(4).

Figure S26.

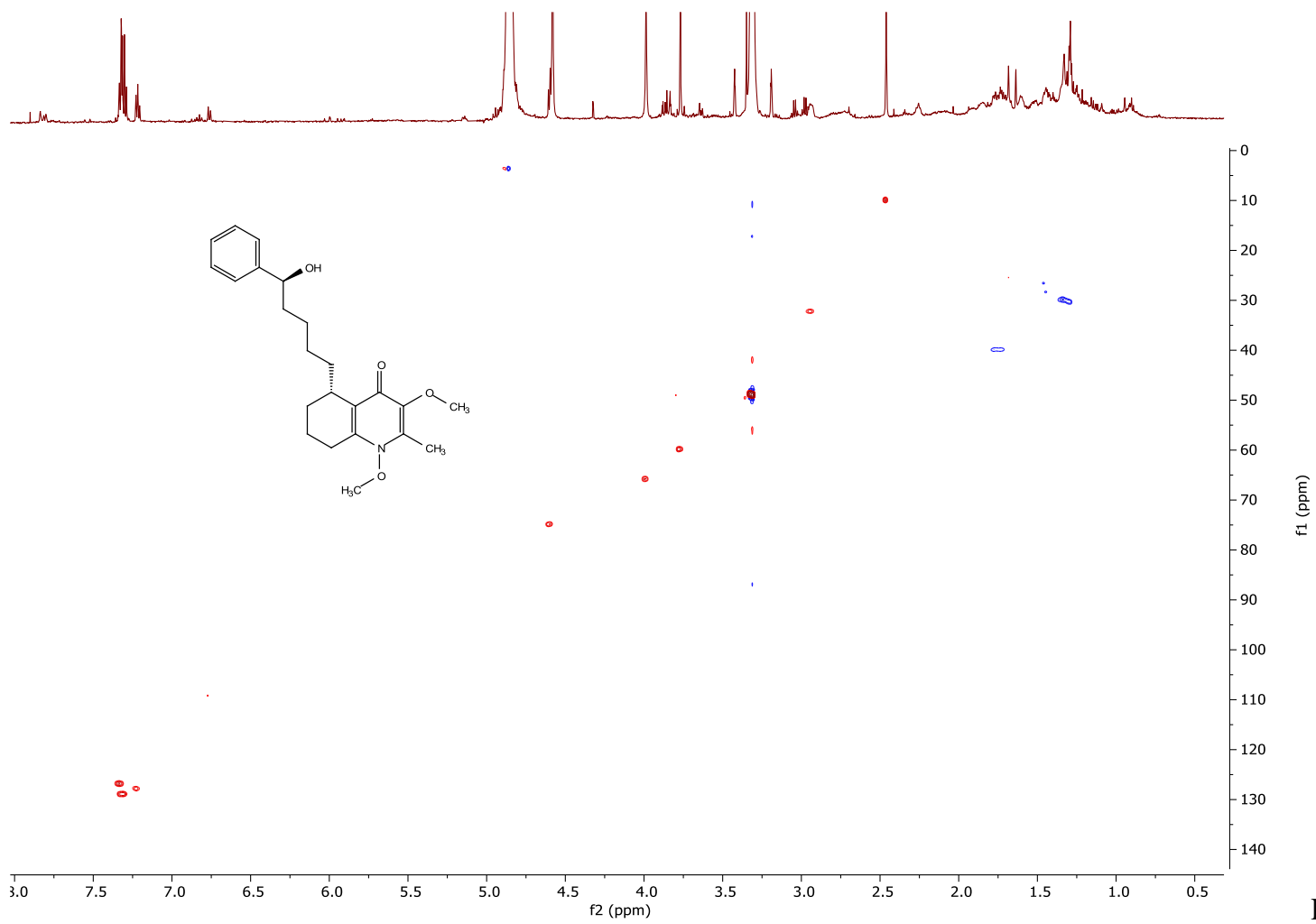

HSQC (600 MHz, methanol- $d_{4}$ ) spectrum of 15-Hydroxywaltherione G (4).

Figure S27. 


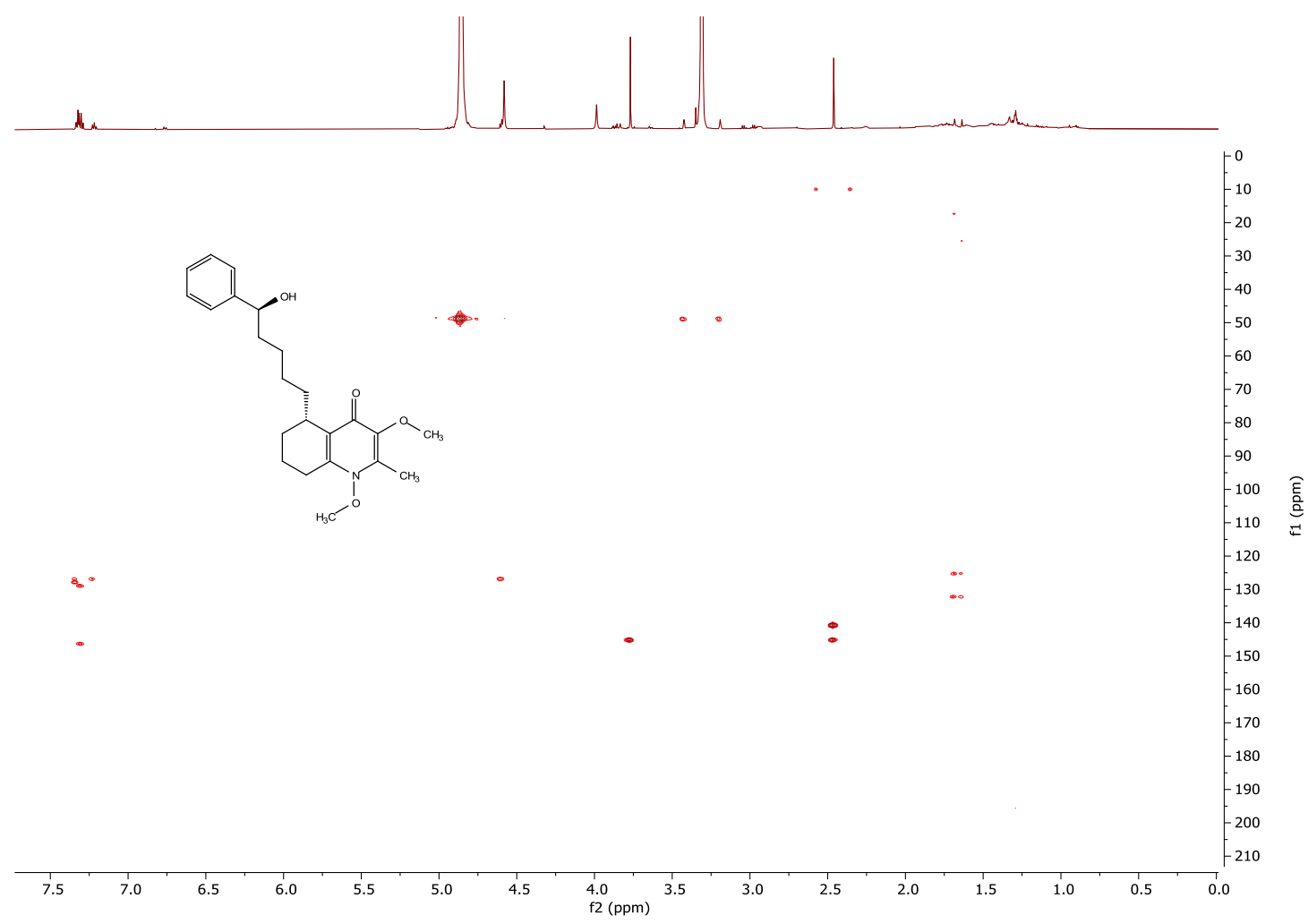

Figure S28. HMBC (600 MHz, methanol- $\left.d_{4}\right)$ spectrum of 15-Hydroxywaltherione G (4).

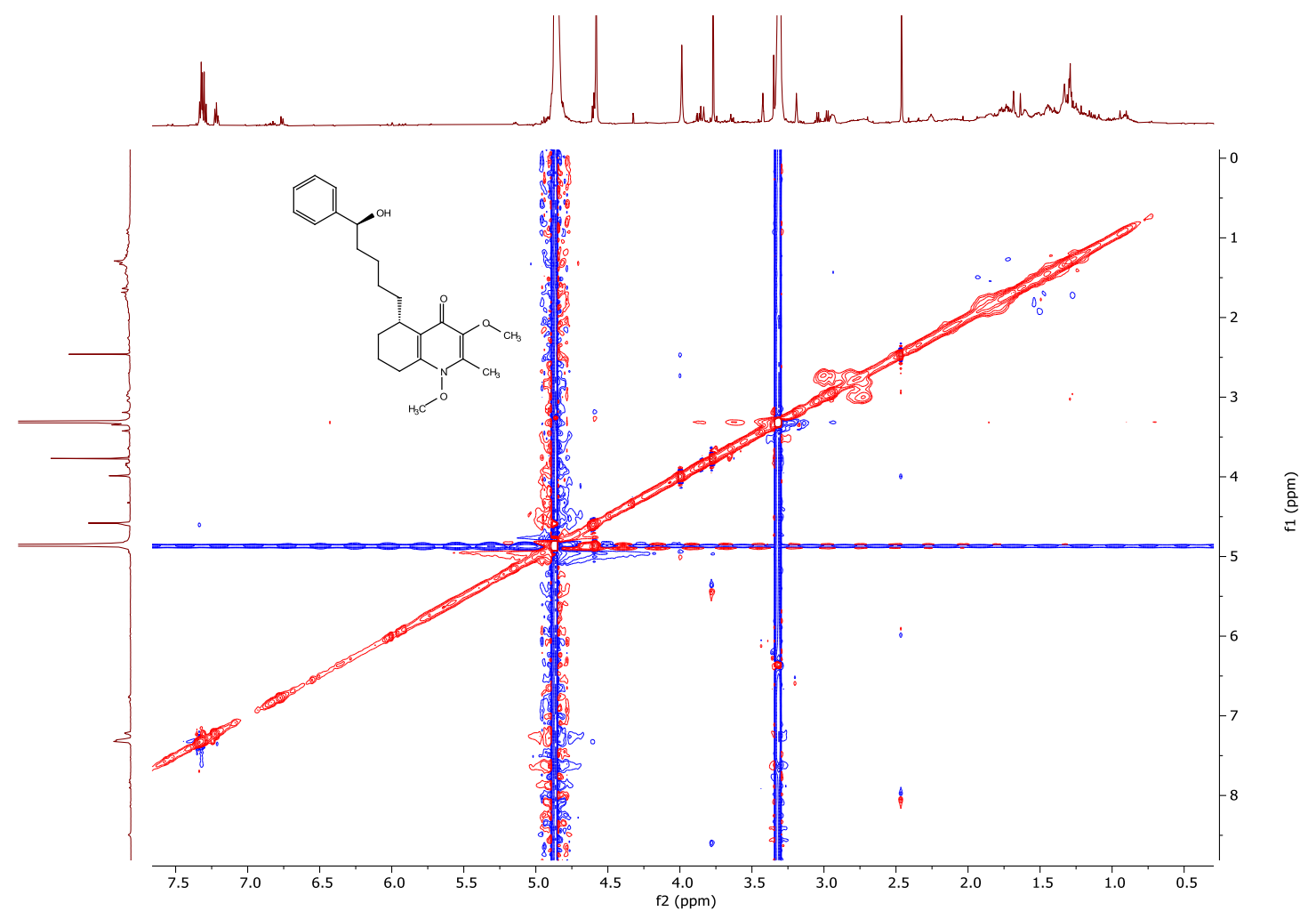

Figure S29. ROESY (600 MHz, methanol- $d_{4}$ ) spectrum of 15-Hydroxywaltherione G (4). 


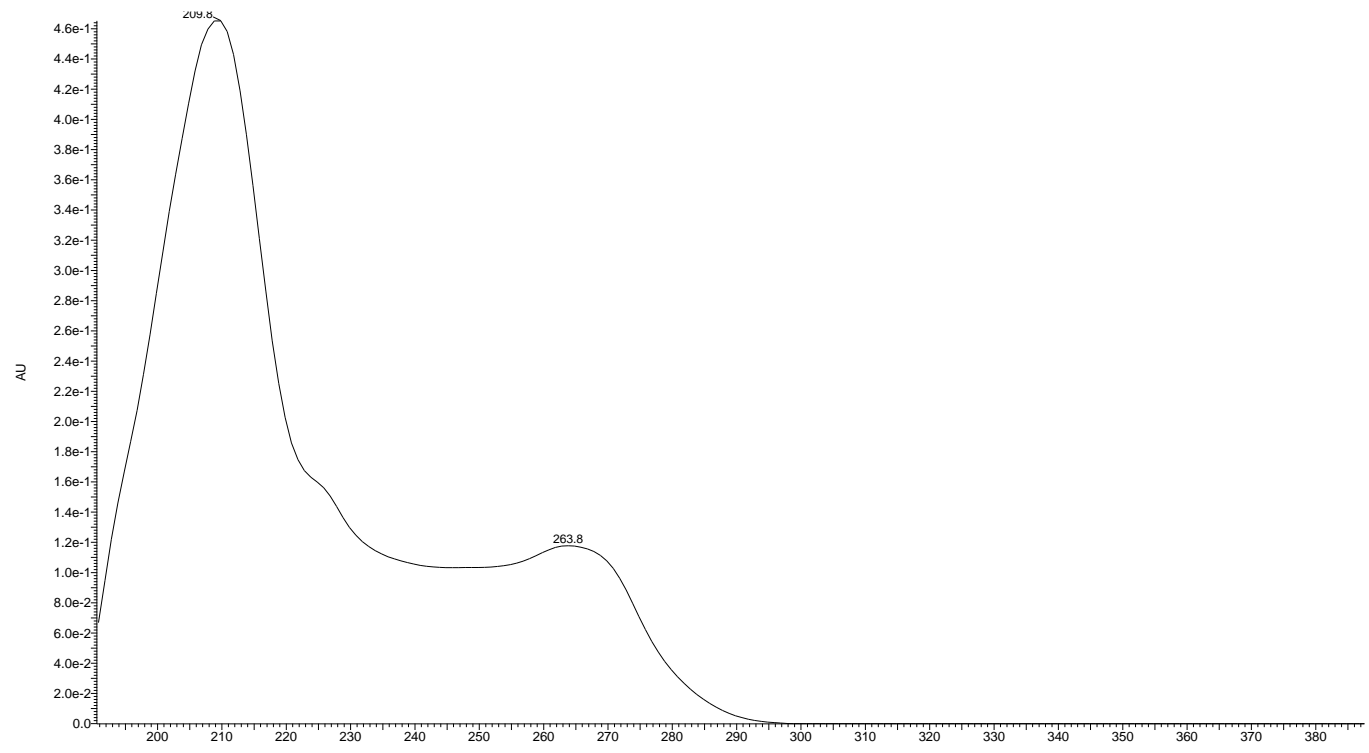

Figure S30. UV spectrum of 8-Hydroxywaltherione M (5).
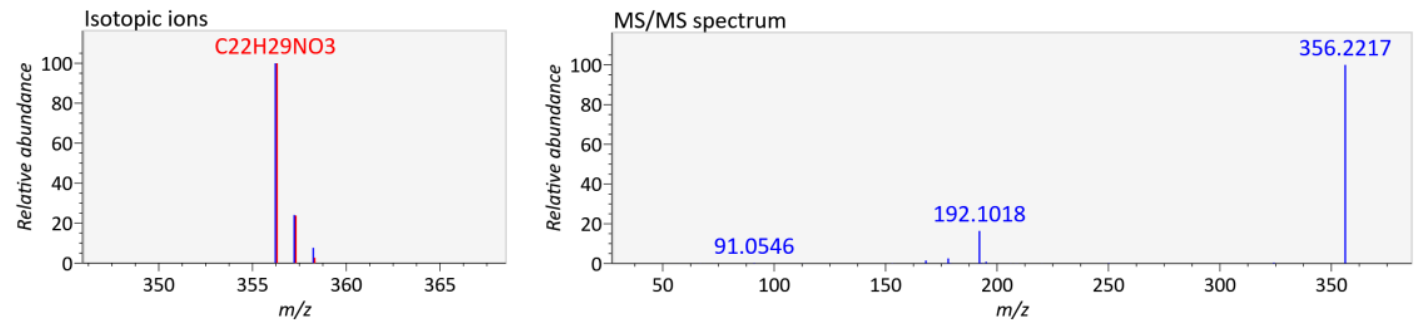

Figure S31. HRESIMS spectrum and MS/MS fragmentation of 8-Hydroxywaltherione M (5) (Q Exactive Focus Hybrid quadripole-orbitrap mass spectrometer, positive mode). 


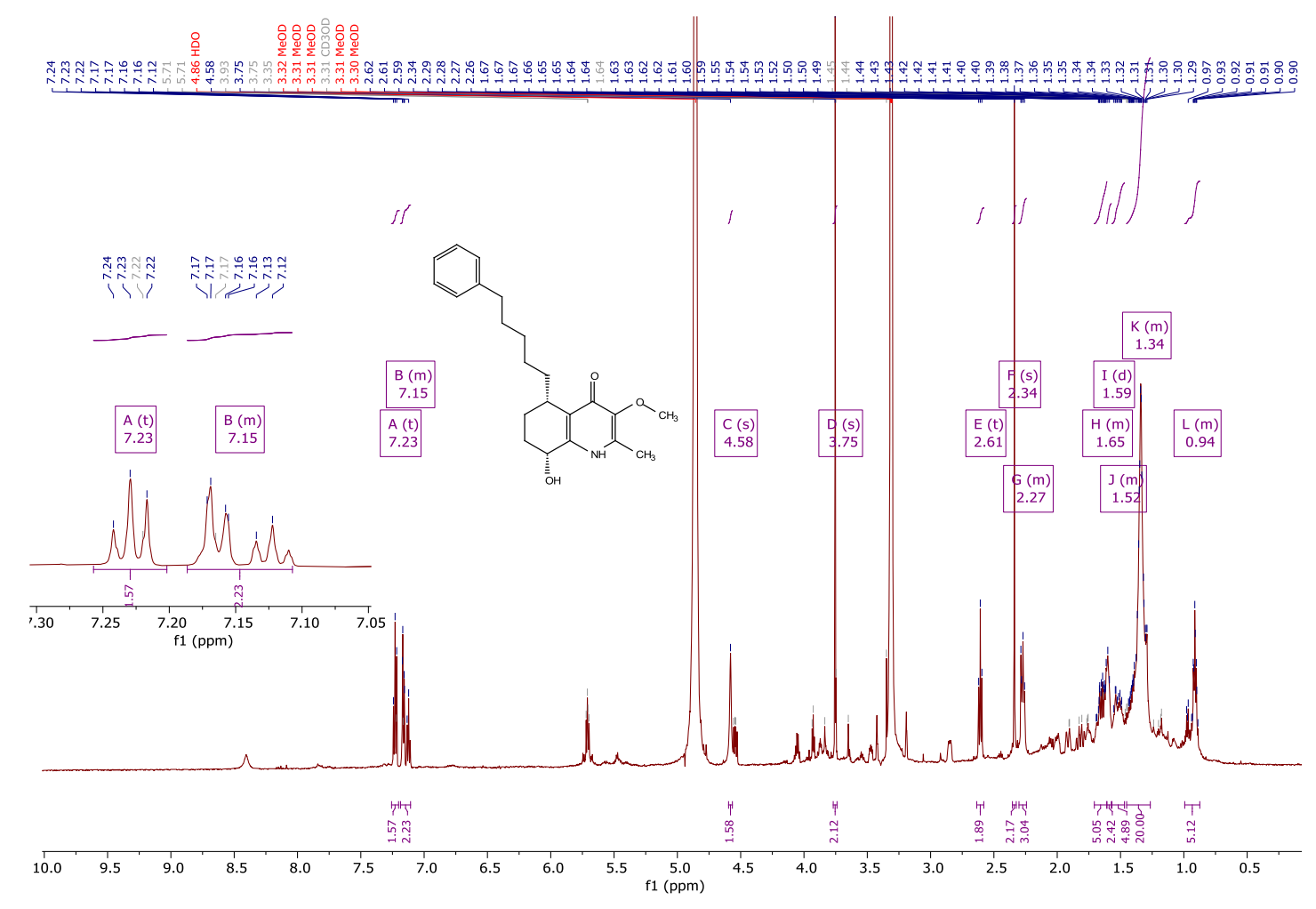

Figure S32. ${ }^{1} \mathrm{H}$ NMR $\left(600 \mathrm{MHz}\right.$, methanol- $\left.d_{4}\right)$ spectrum of 8-Hydroxywaltherione M (5).

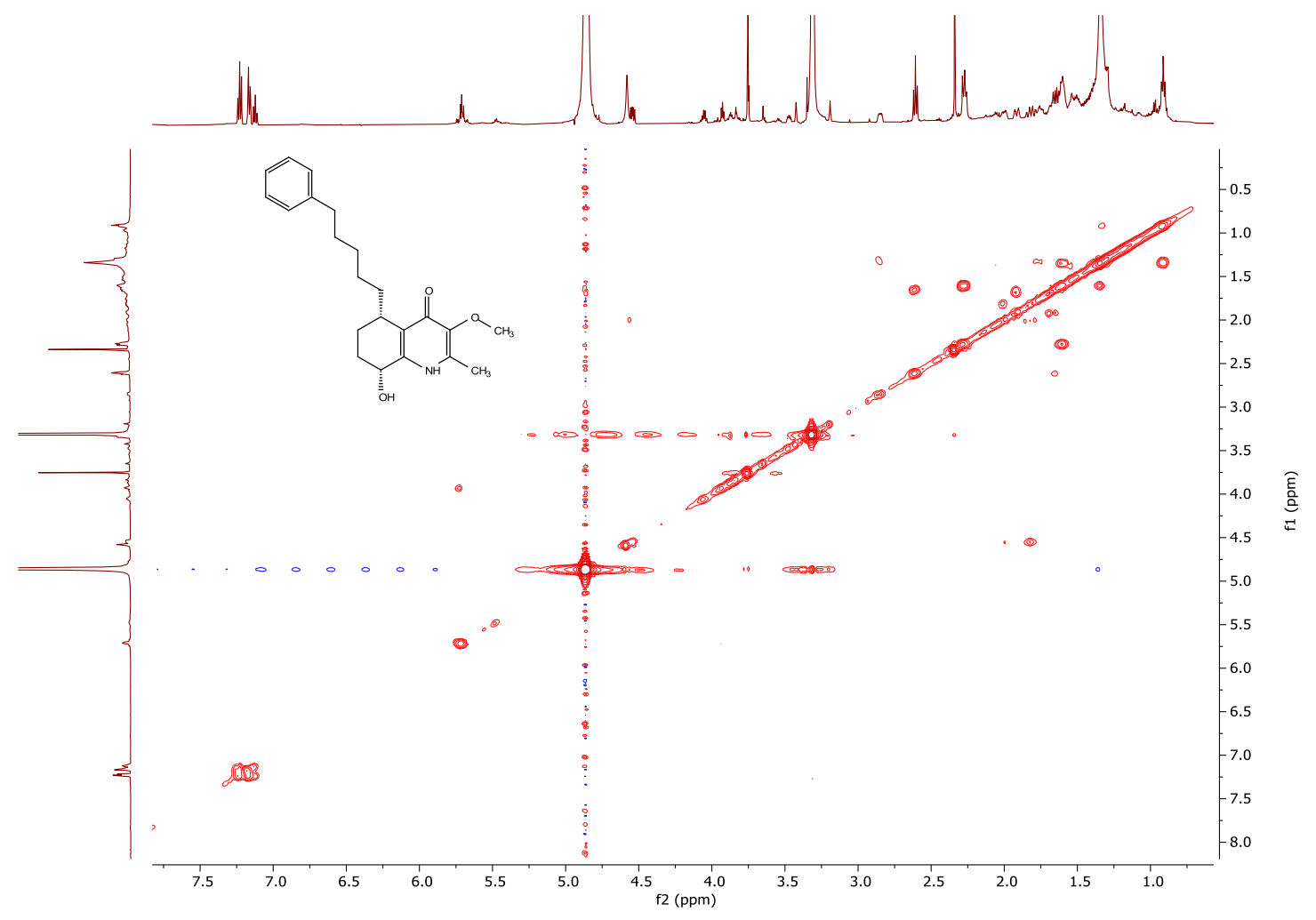

Figure S33. COSY (600 MHz, methanol- $d_{4}$ ) spectrum of 8-Hydroxywaltherione M (5). 


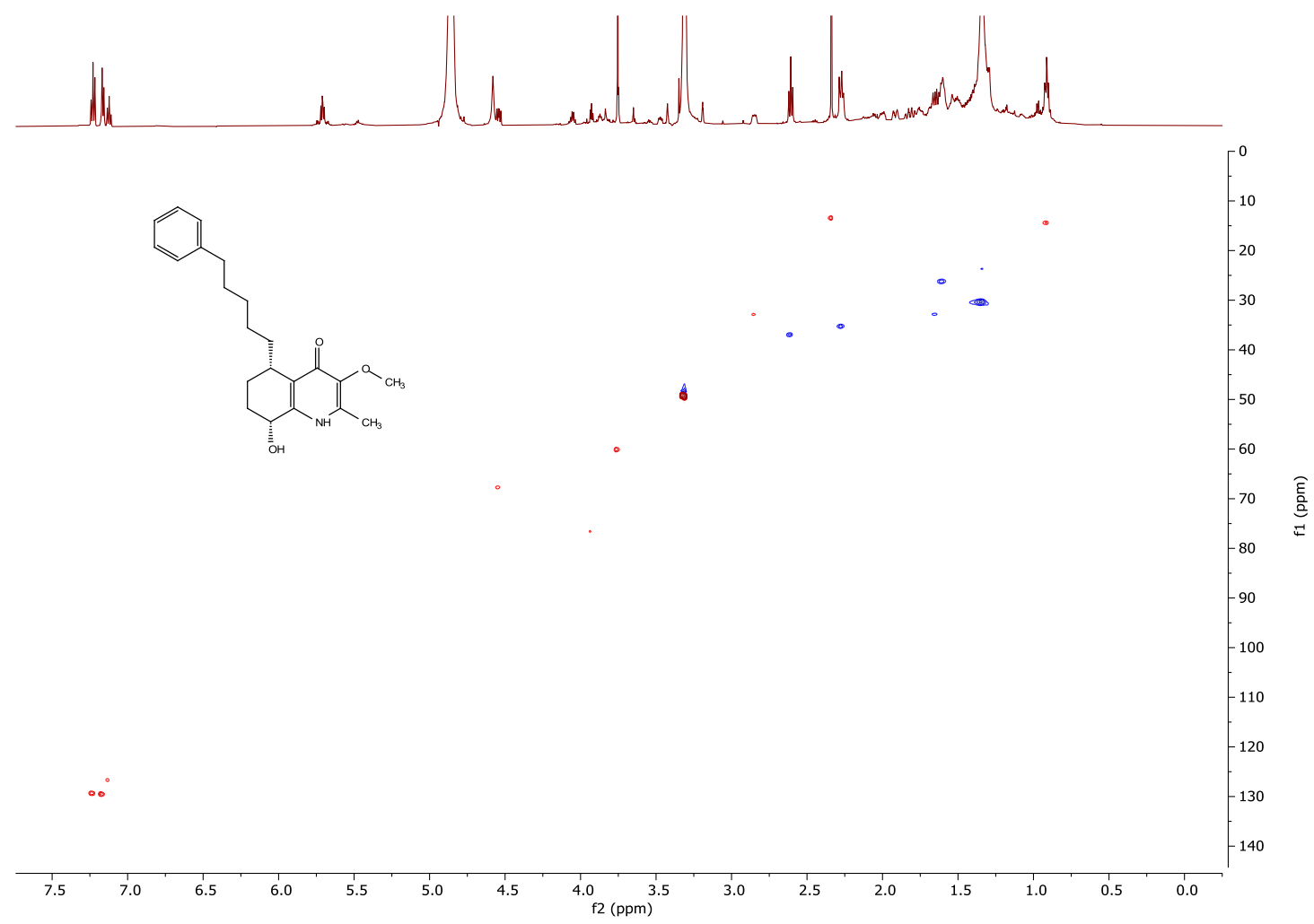

Figure S34. HSQC (600 MHz, methanol- $d_{4}$ ) spectrum of 8-Hydroxywaltherione M (5).

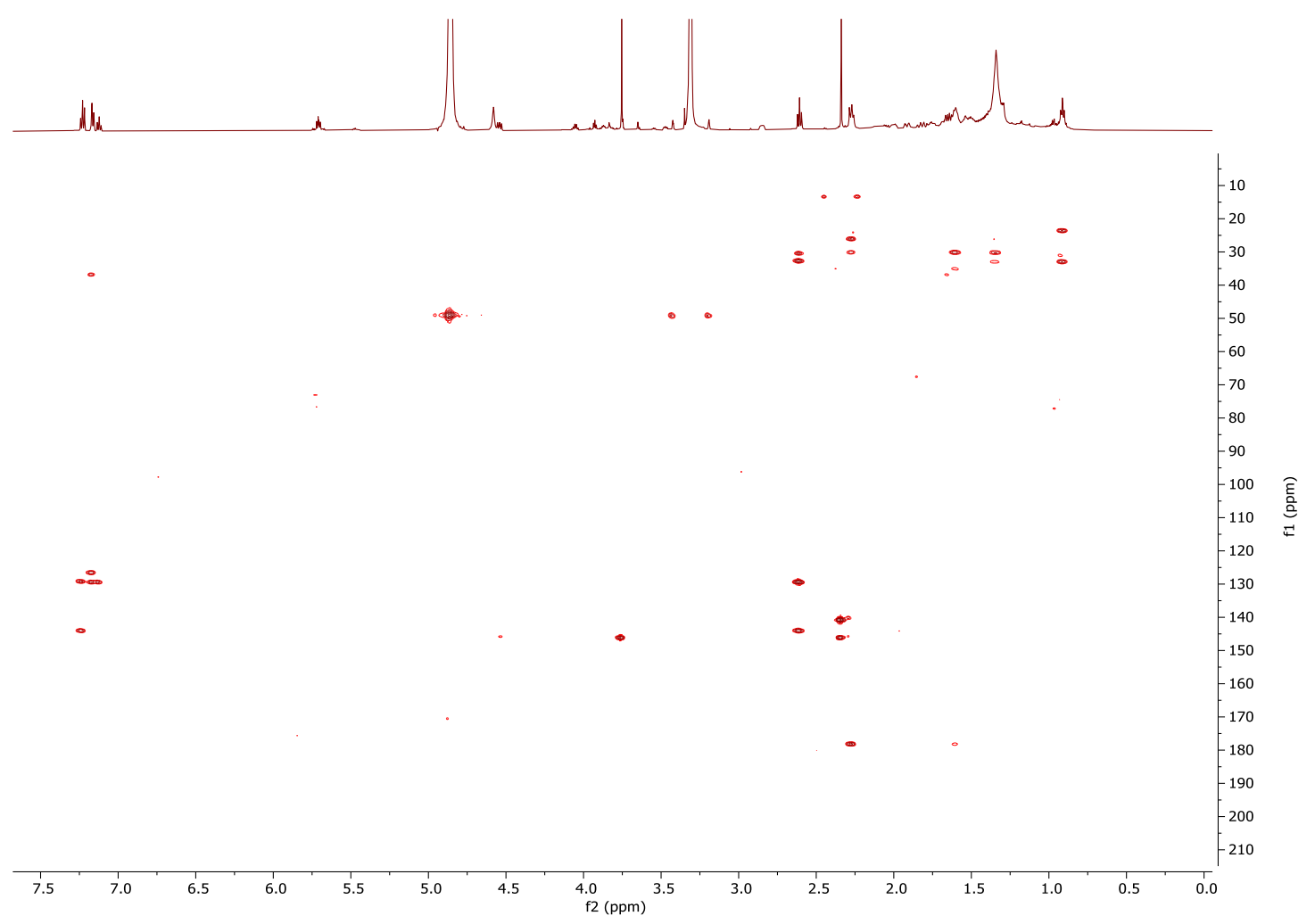

Figure S35. HMBC (600 MHz, methanol- $\left.d_{4}\right)$ spectrum of 8-Hydroxywaltherione M (5). 


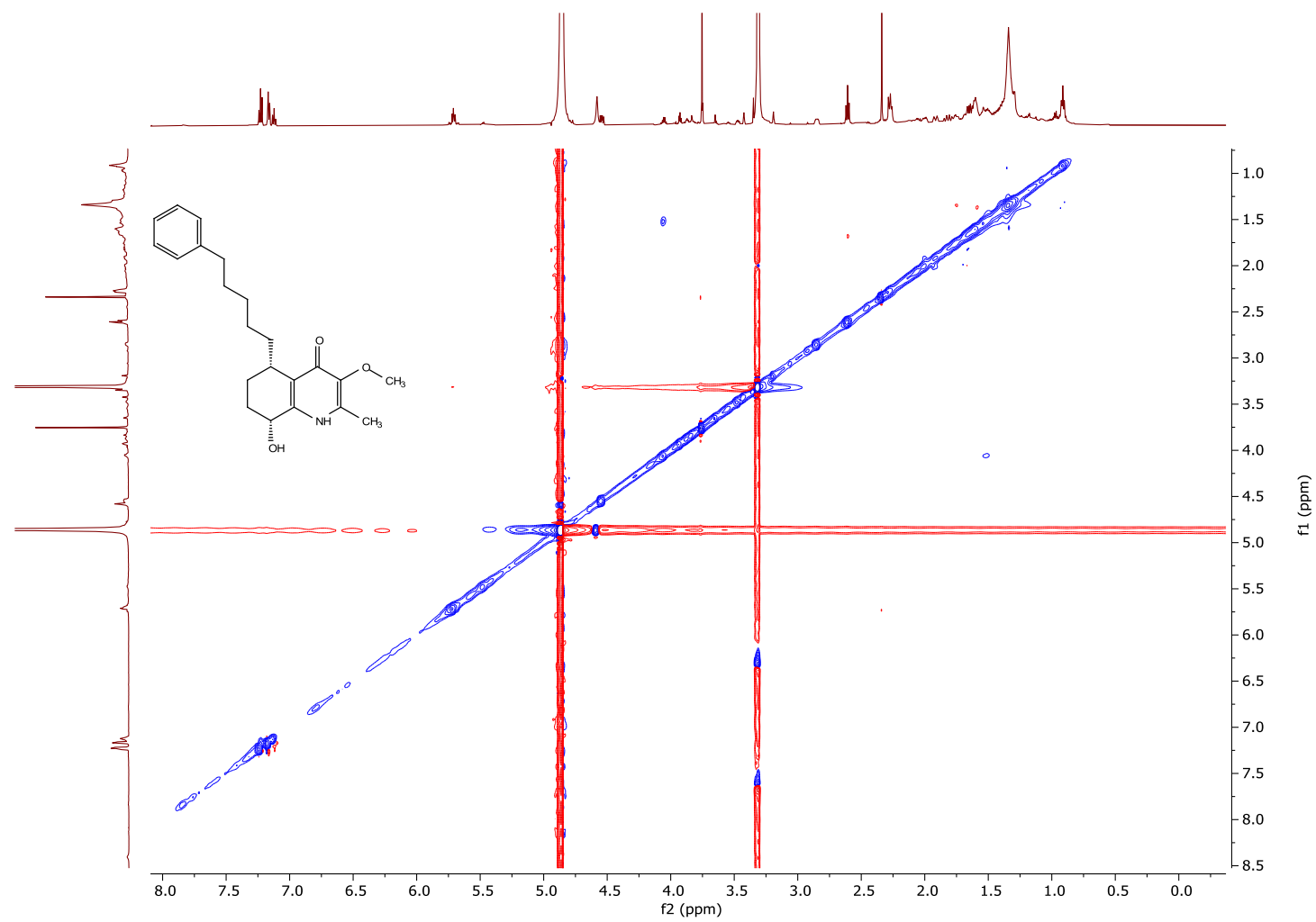

Figure S36. ROESY (600 MHz, methanol- $d_{4}$ ) spectrum of 8-Hydroxywaltherione M (5).

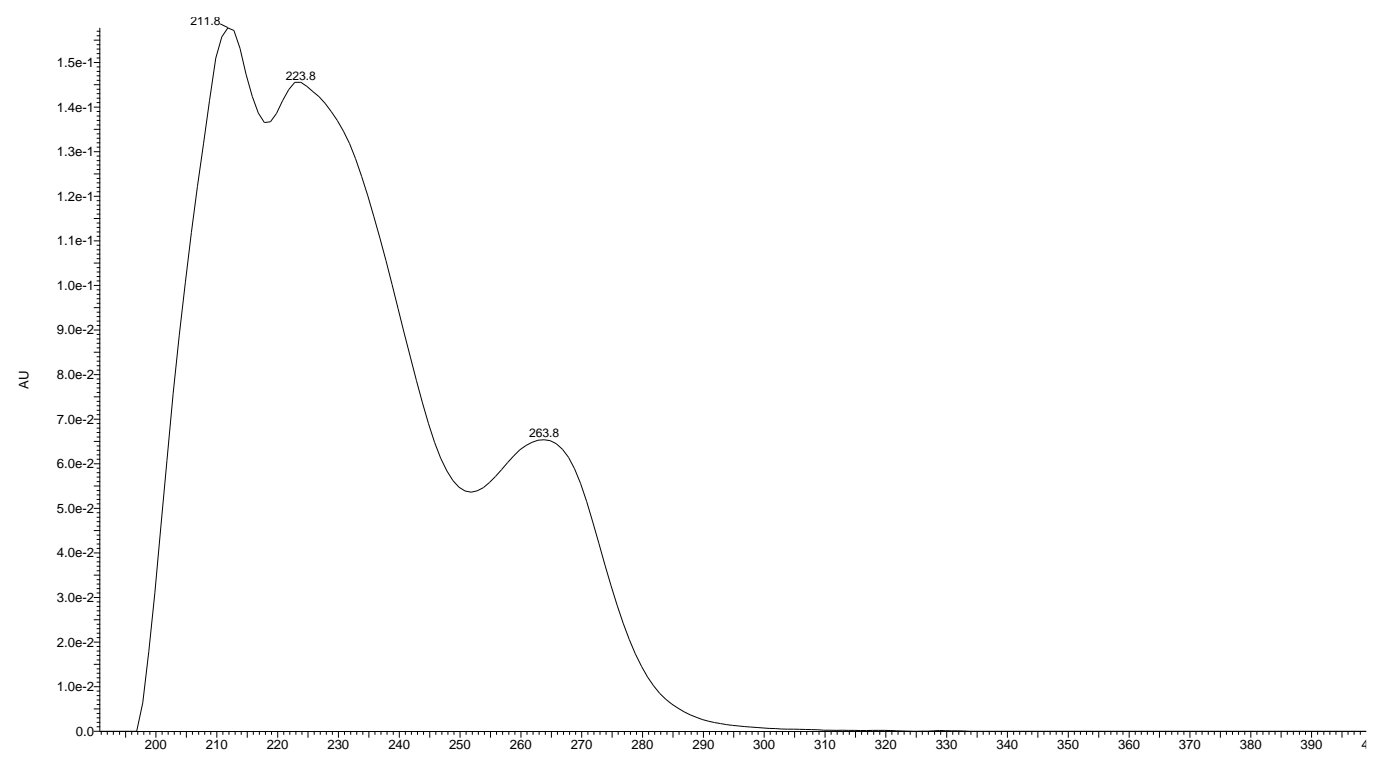

Figure S37. UV spectrum of Waltherione S (6). 

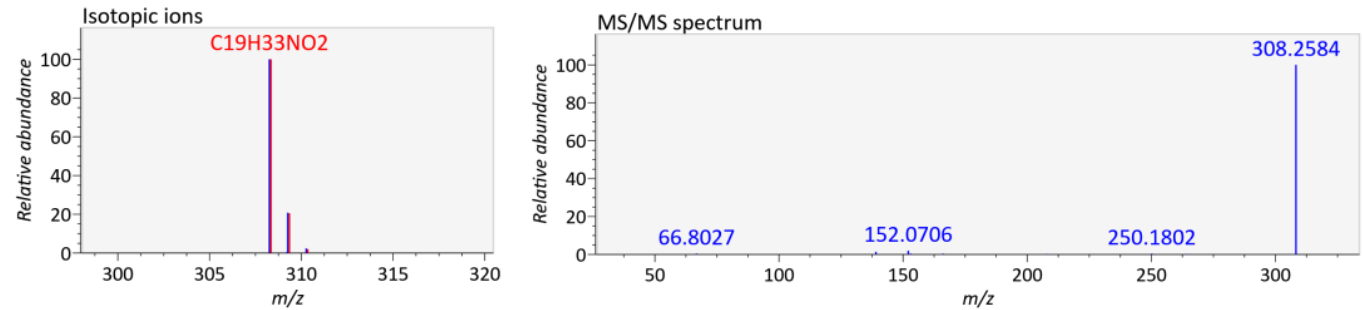

Figure S38. HRESIMS spectrum and MS/MS fragmentation of Waltherione S (6) (Q Exactive Focus Hybrid quadripole-orbitrap mass spectrometer, positive mode).

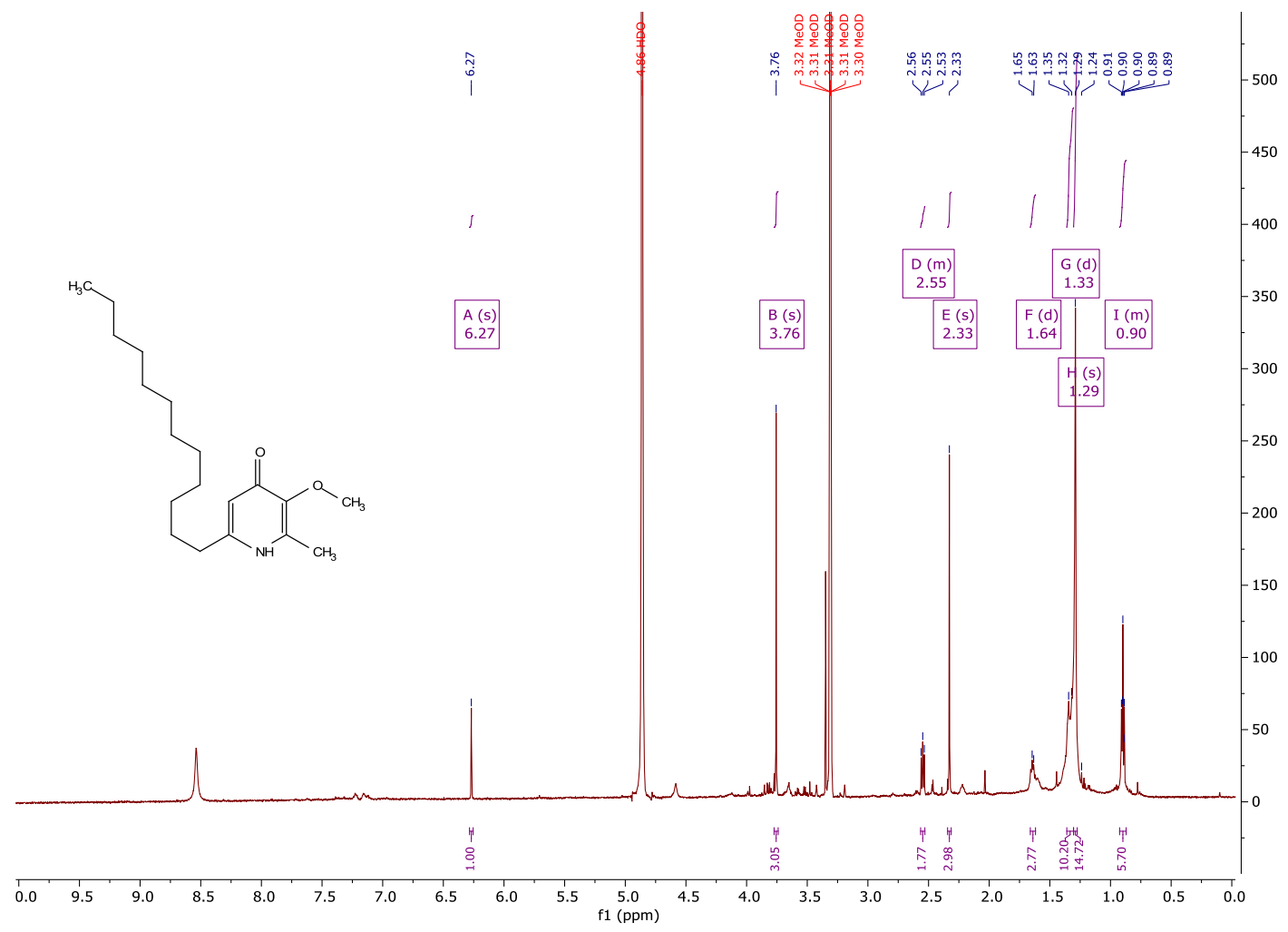

Figure S39. ${ }^{1} \mathrm{H}$ NMR (600 MHz, methanol- $\left.d_{4}\right)$ spectrum of Waltherione S (6). 


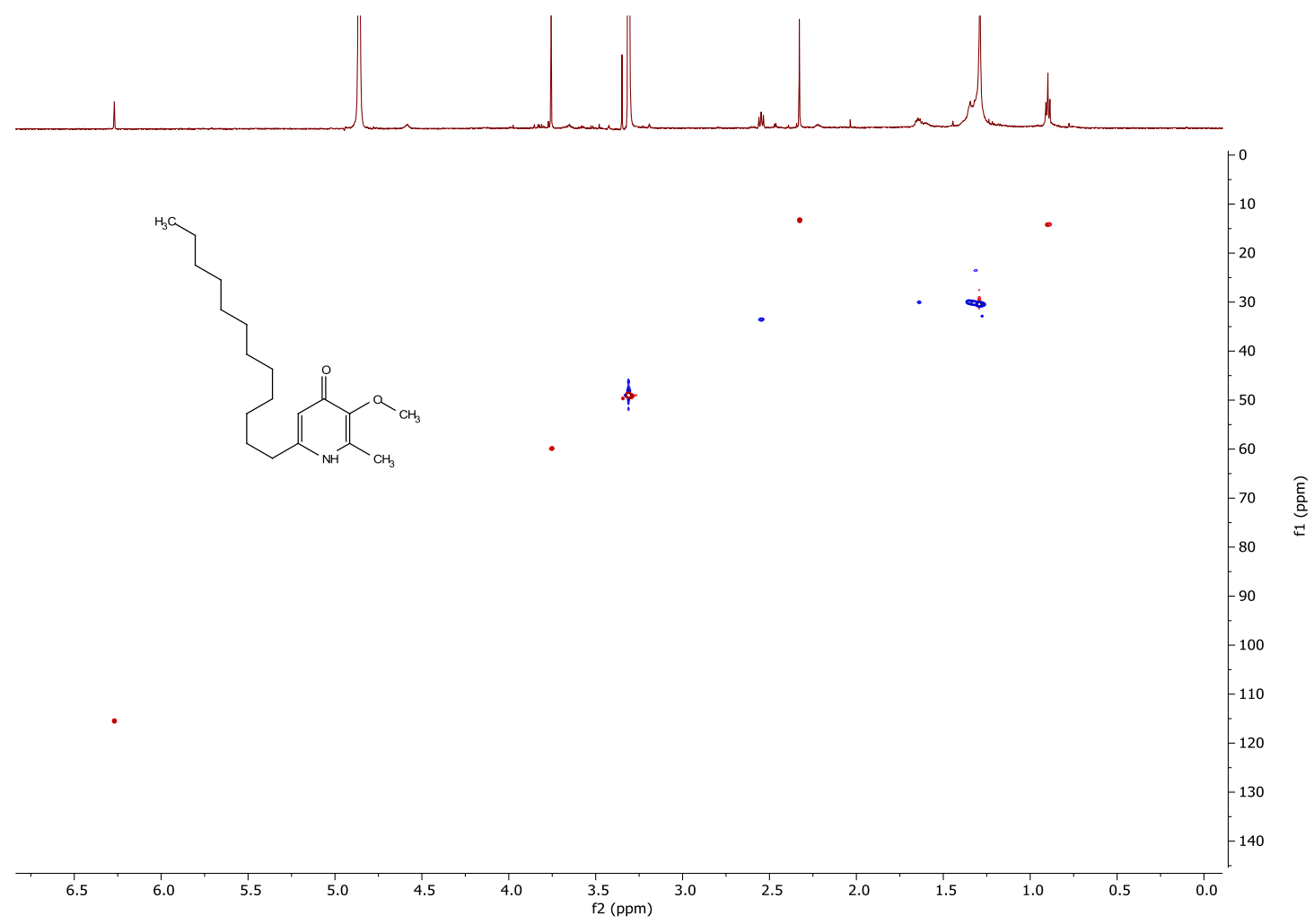

Figure S40. HSQC (600 MHz, methanol- $\left.d_{4}\right)$ spectrum of Waltherione S (6).

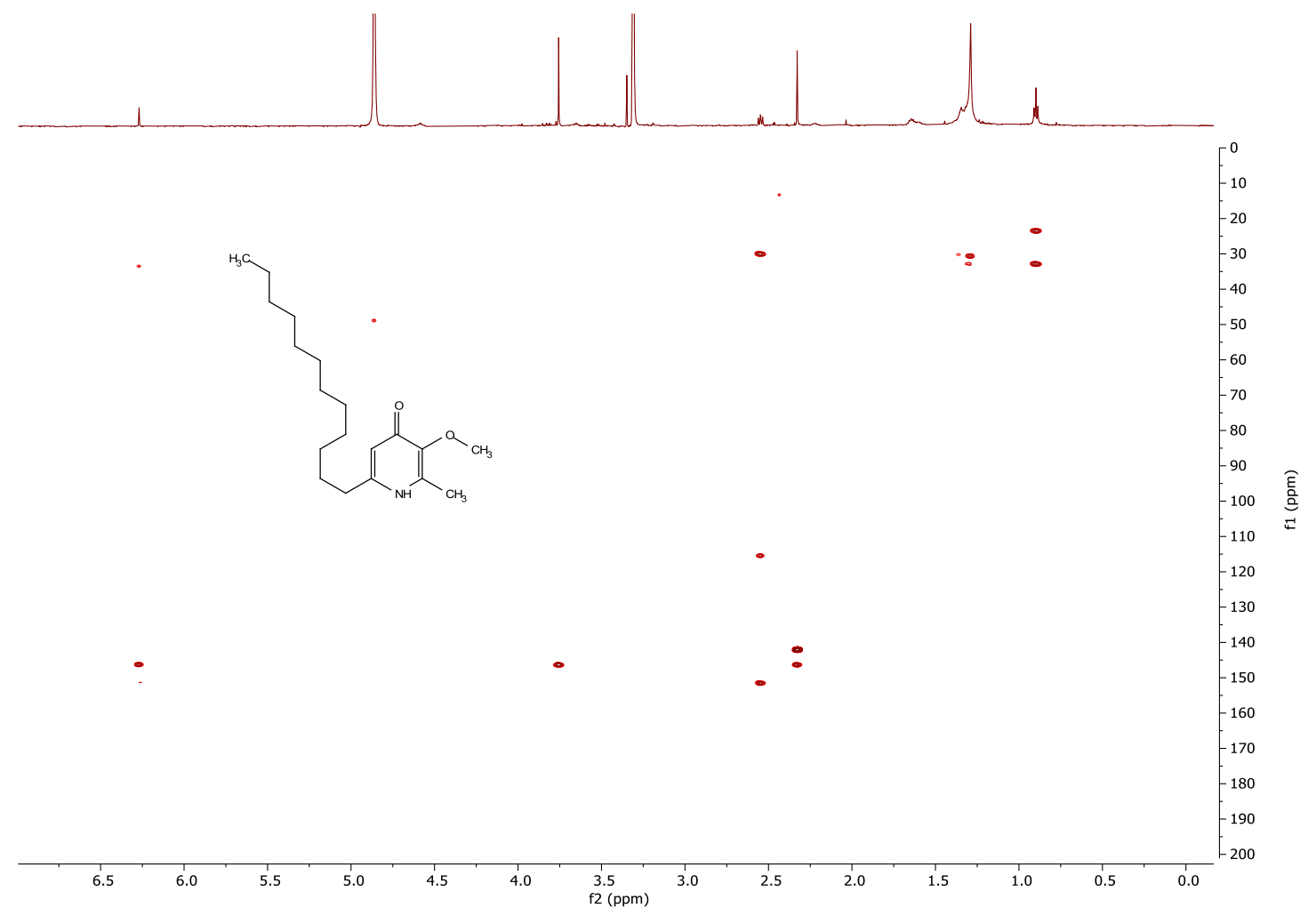

Figure S41. HMBC (600 MHz, methanol- $\left.d_{4}\right)$ spectrum of Waltherione $\mathrm{S}(\mathbf{6})$. 


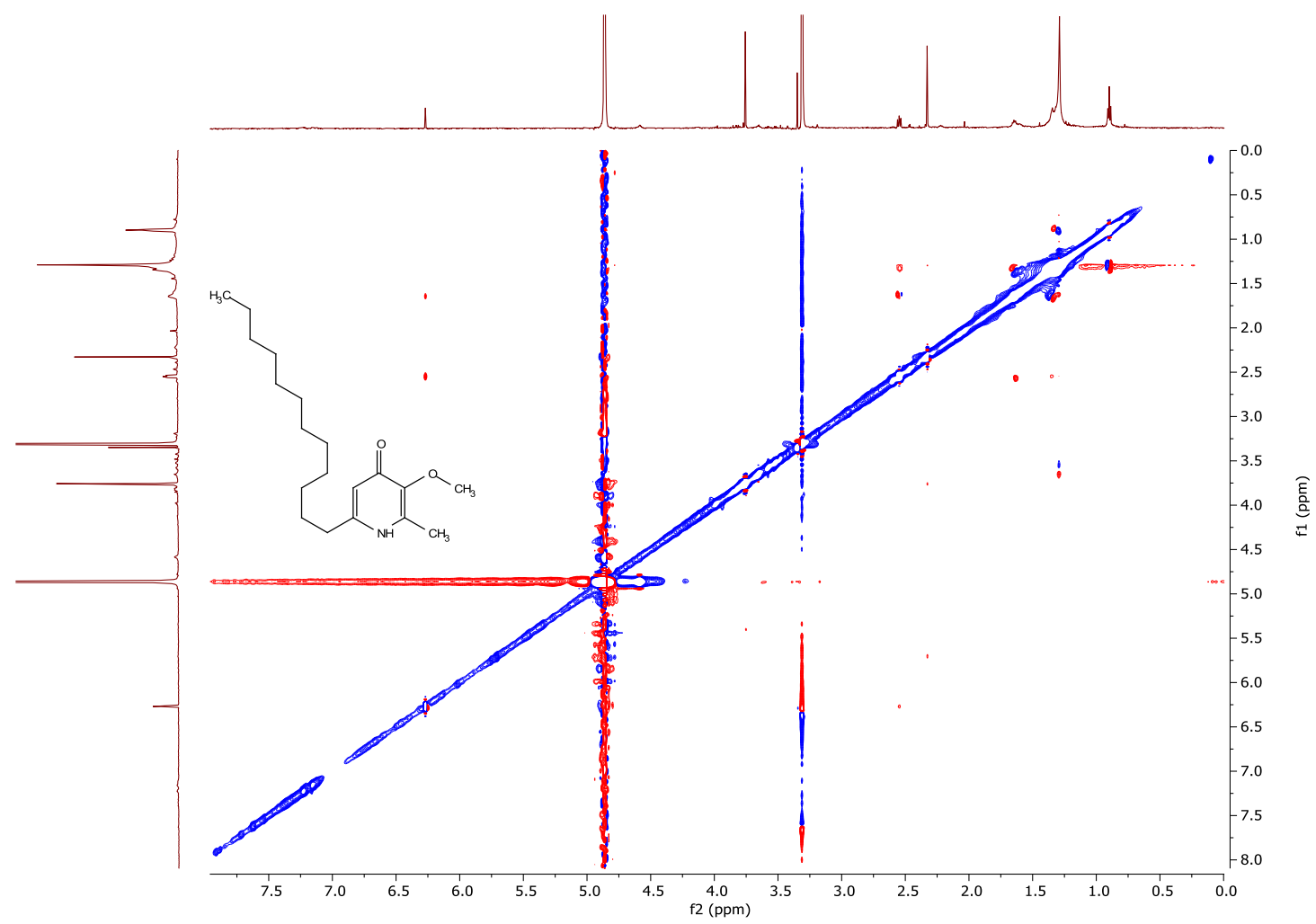

Figure S42. ROESY (600 MHz, methanol- $\left.d_{4}\right)$ spectrum of Waltherione S (6).

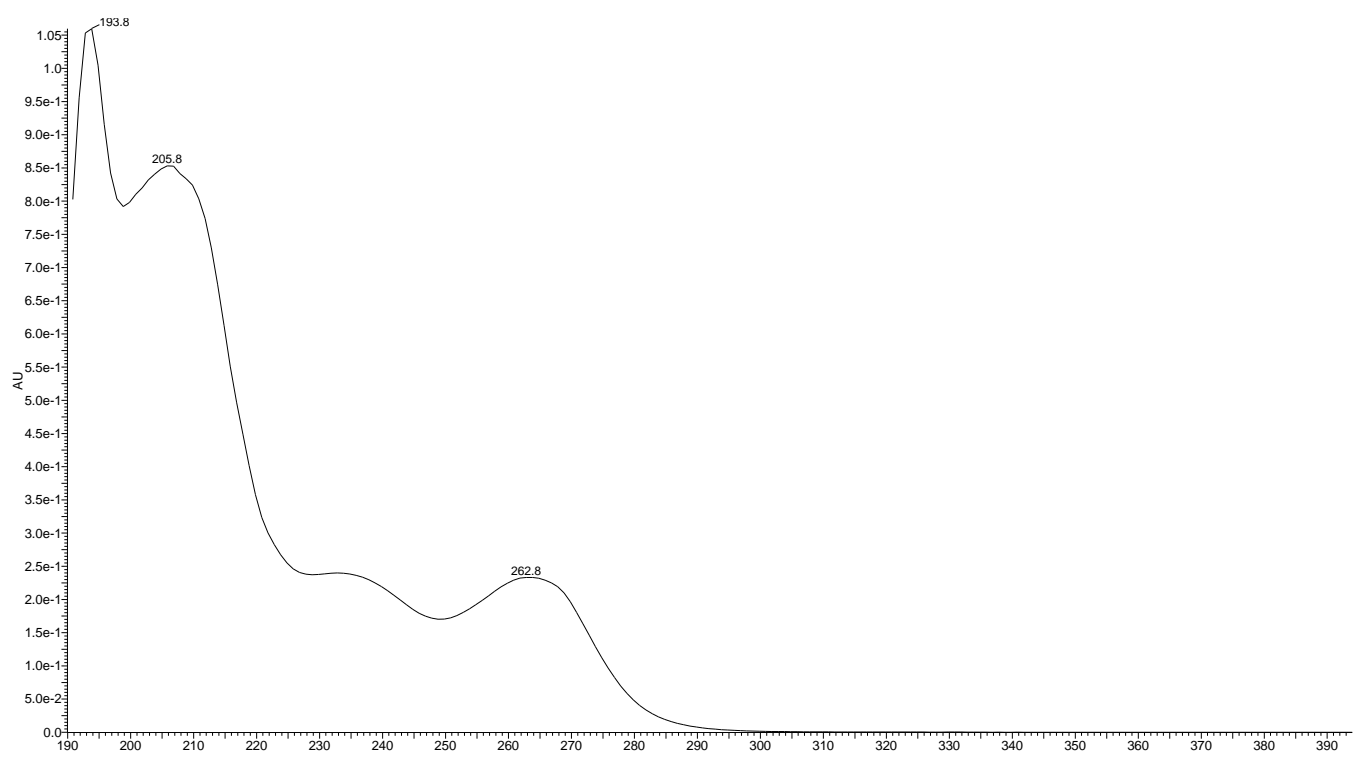

Figure S43. UV spectrum of Waltherione T (7). 

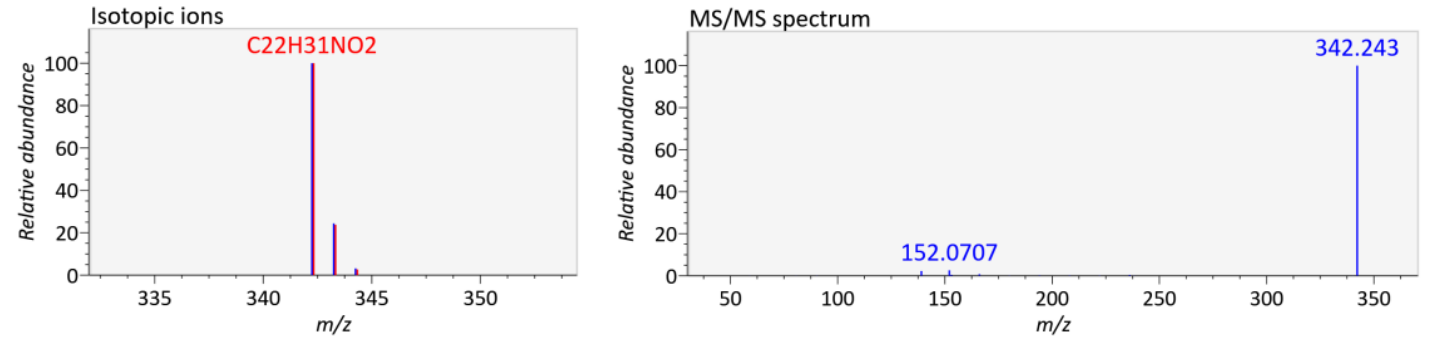

Figure S44. HRESIMS spectrum and MS/MS fragmentation of Waltherione T (7) (Q Exactive Focus Hybrid quadripole-orbitrap mass spectrometer, positive mode).

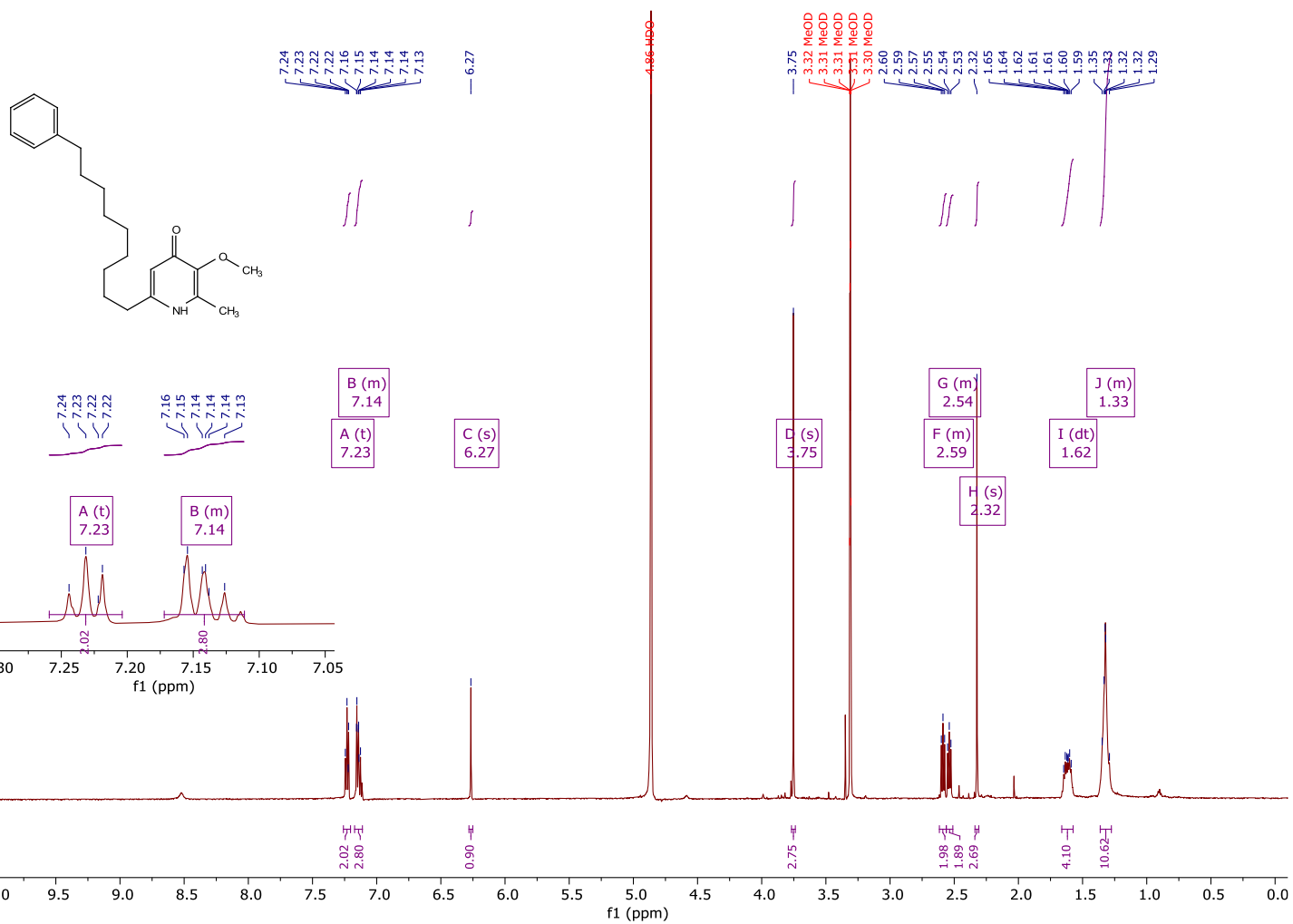

Figure S45. ${ }^{1} \mathrm{H}$ NMR (600 MHz, methanol- $\left.d_{4}\right)$ spectrum of Waltherione T (7). 


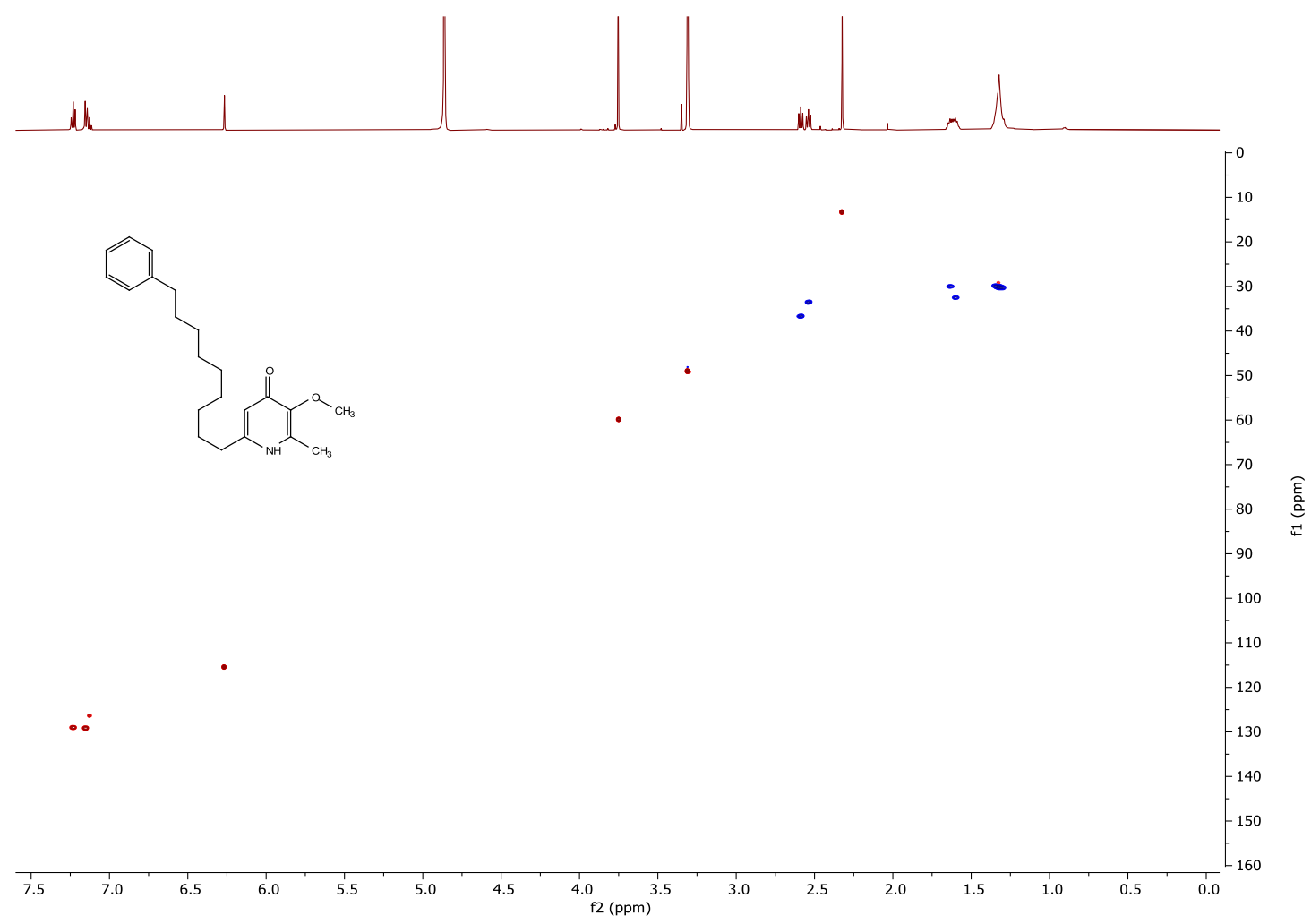

Figure S46. HSQC (600 MHz, methanol- $\left.d_{4}\right)$ spectrum of Waltherione T (7).

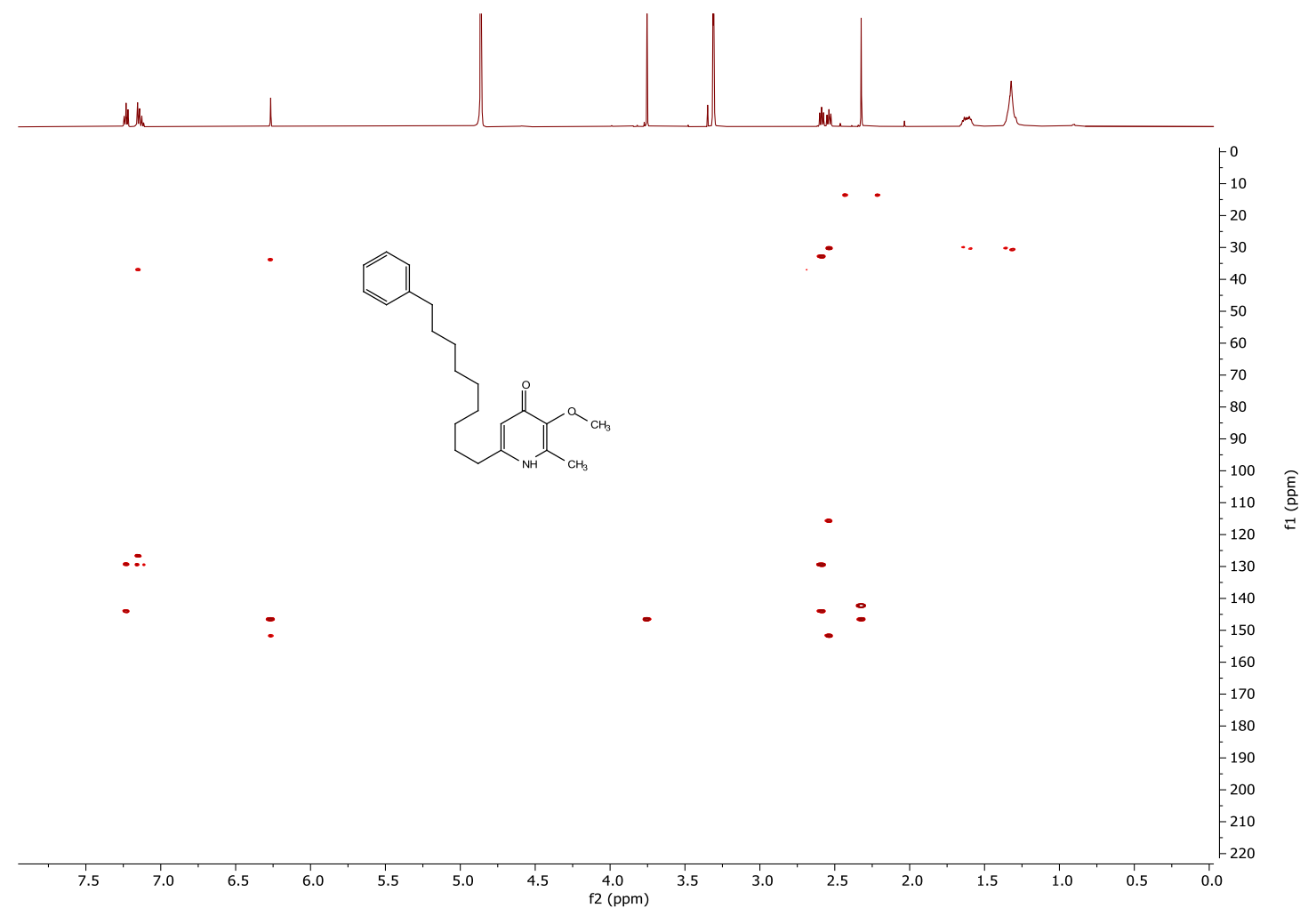

Figure S47. HMBC (600 MHz, methanol- $\left.d_{4}\right)$ spectrum of Waltherione T (7). 


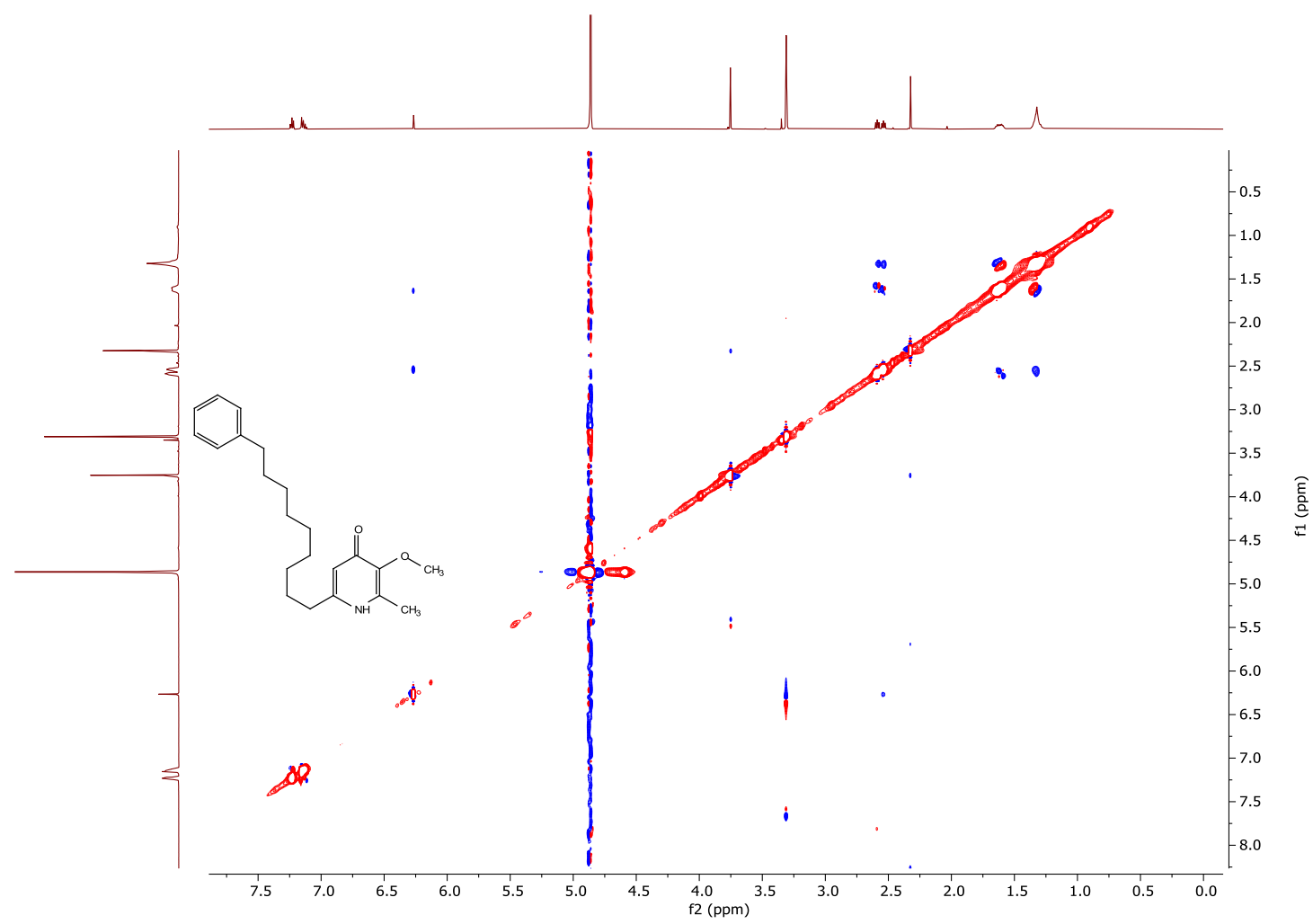

Figure S48. ROESY (600 MHz, methanol- $d_{4}$ ) spectrum of Waltherione T (7).

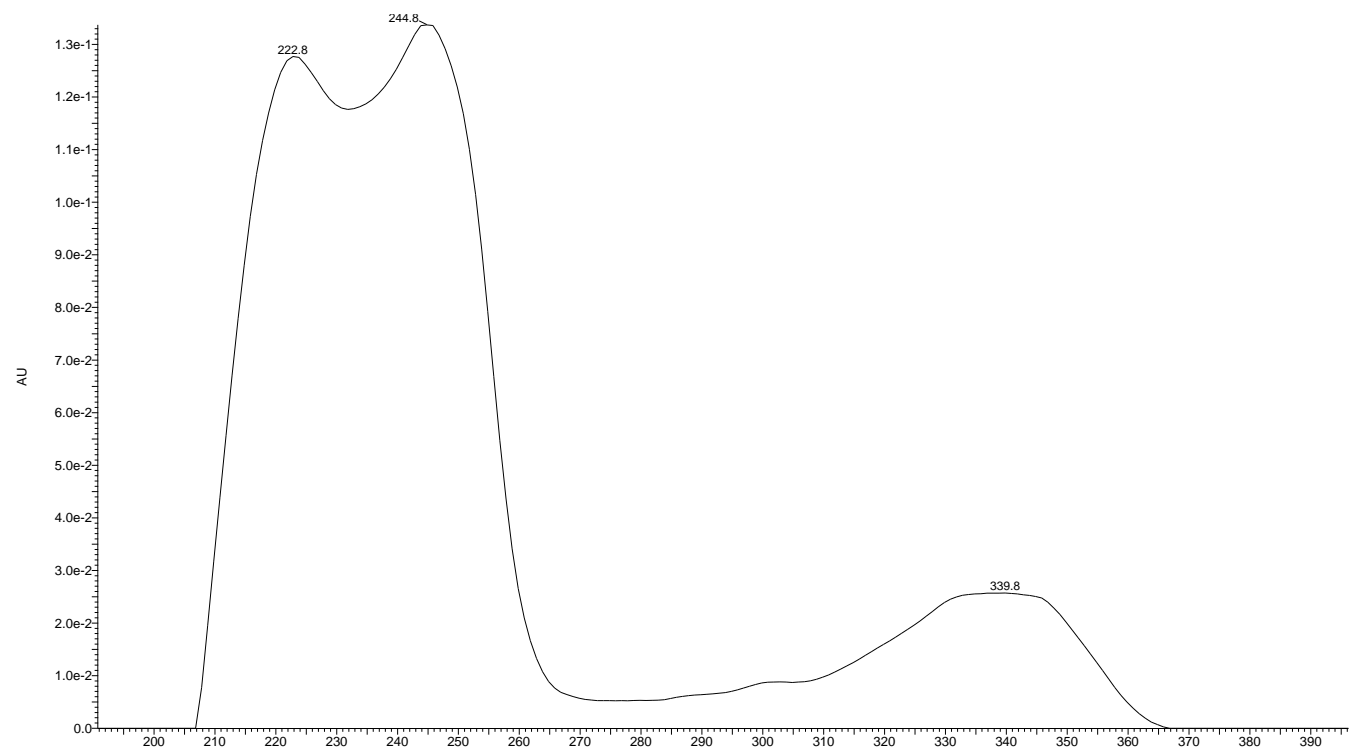

Figure S49. UV spectrum of 2-Hydroxymethylwaltherione C (8). 

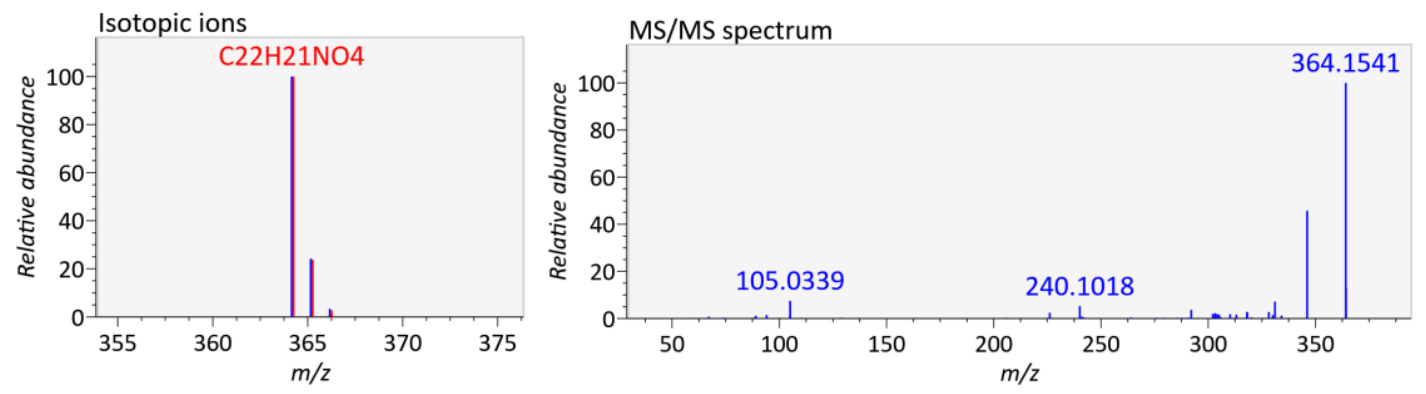

Figure S50. HRESIMS spectrum and MS/MS fragmentation of 2-Hydroxymethylwaltherione C (8) (Q Exactive Focus Hybrid quadripole-orbitrap mass spectrometer, positive mode).

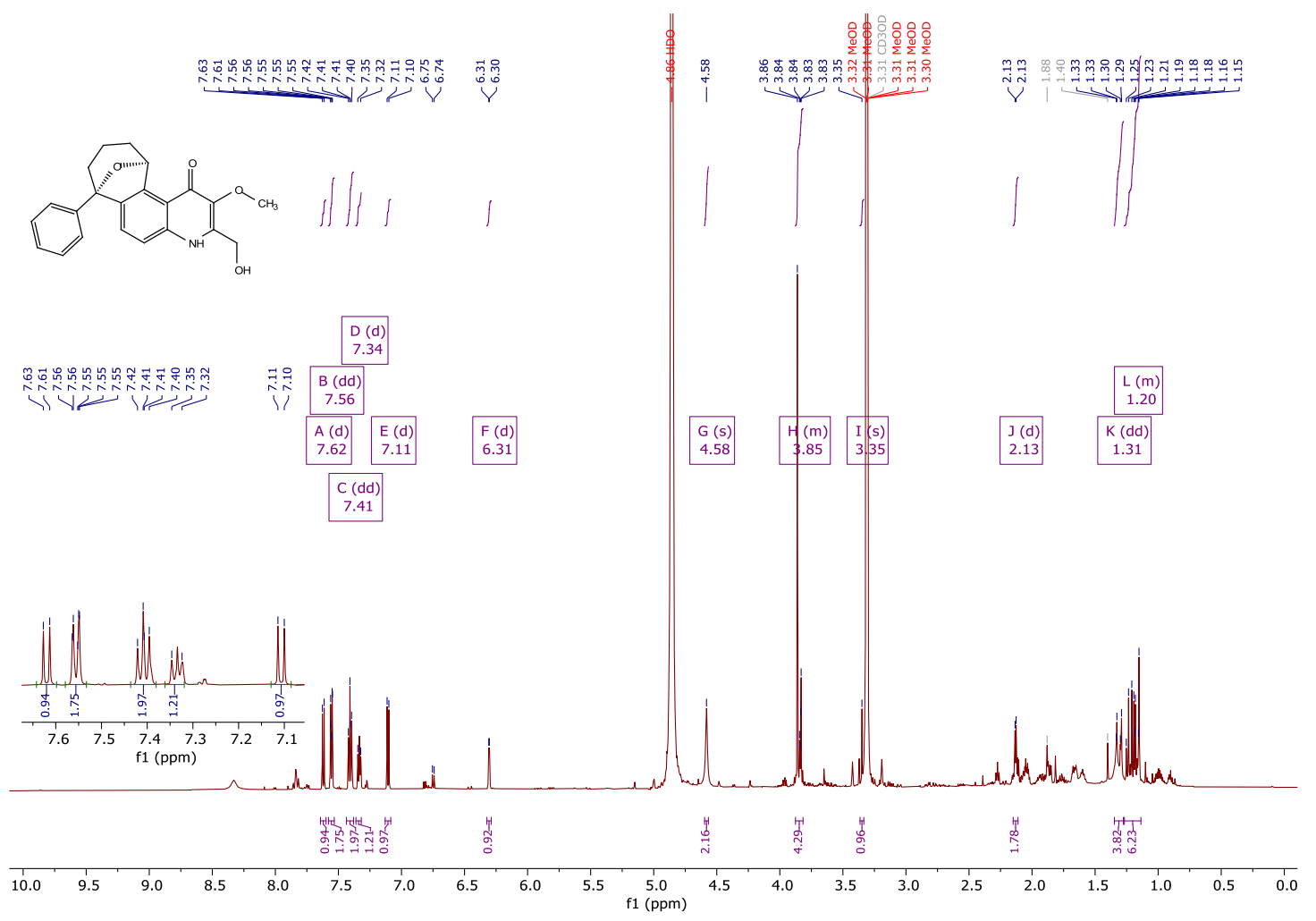

Figure S51. ${ }^{1} \mathrm{H}$ NMR $\left(600 \mathrm{MHz}\right.$, methanol- $\left.d_{4}\right)$ spectrum of 2-Hydroxymethylwaltherione $\mathrm{C}(\mathbf{8})$. 


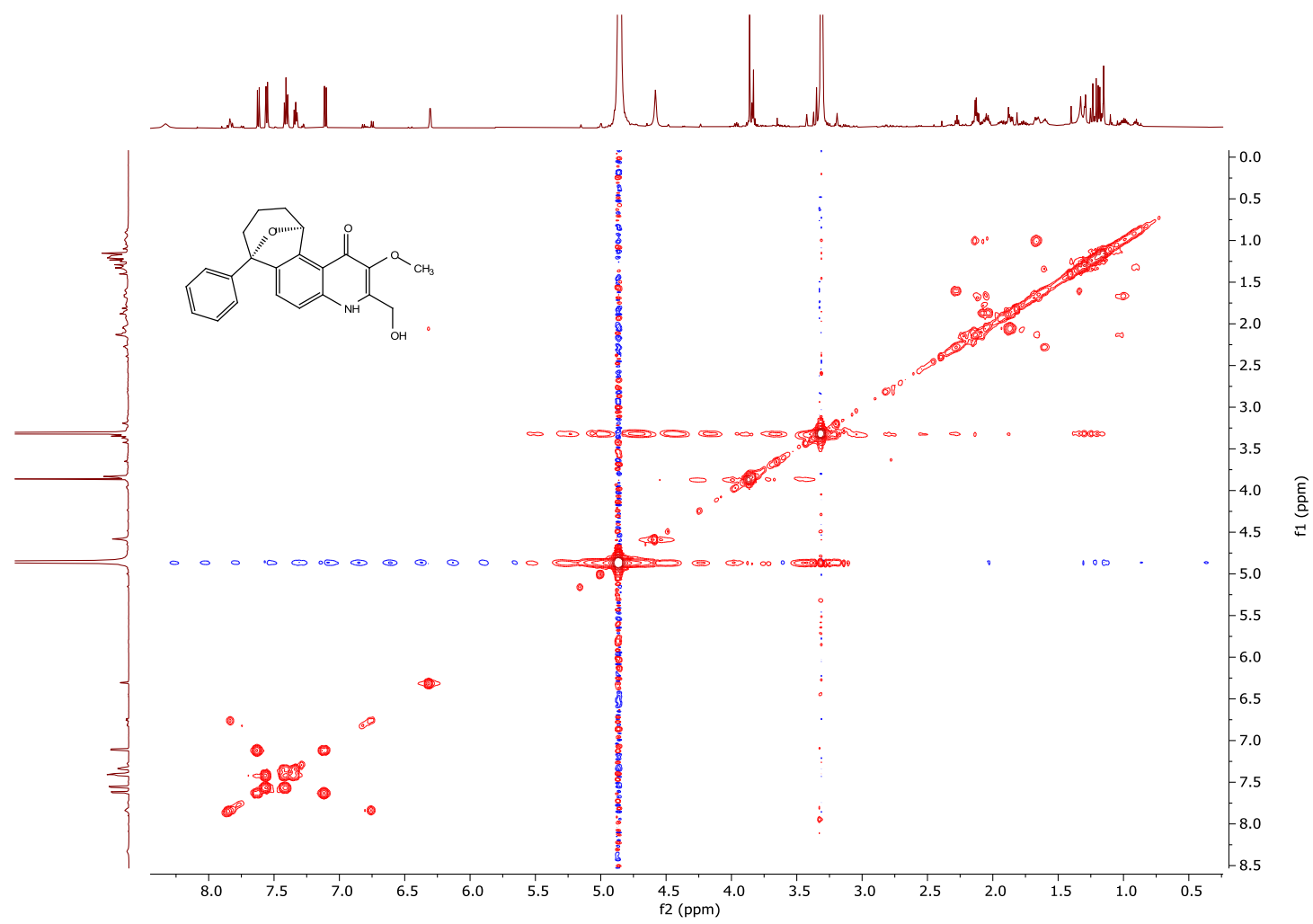

Figure S52. COSY (600 MHz, methanol- $\left.d_{4}\right)$ spectrum of 2-Hydroxymethylwaltherione C (8).

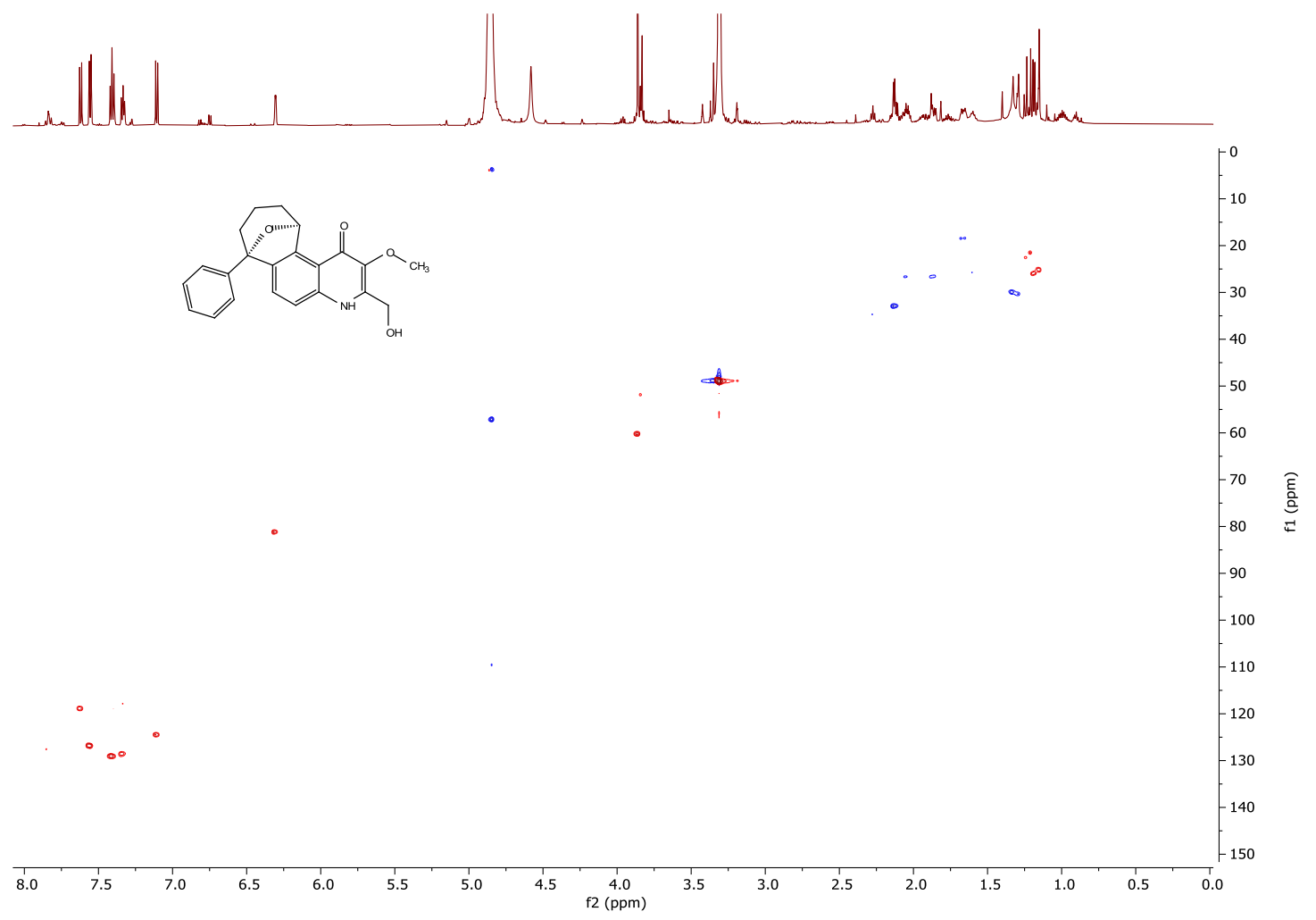

Figure S53. HSQC (600 MHz, methanol- $\left.d_{4}\right)$ spectrum of 2-Hydroxymethylwaltherione C (8). 


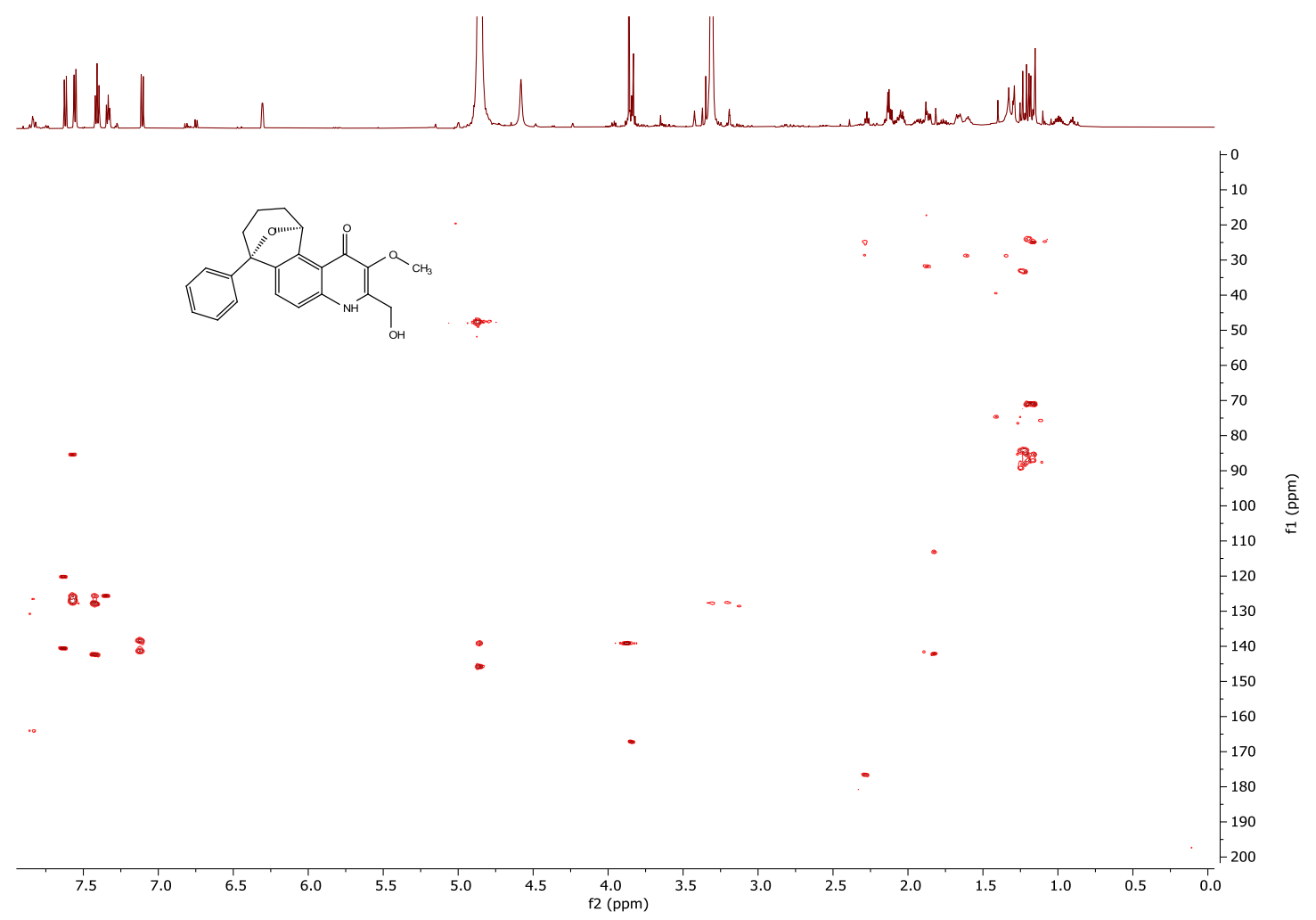

Figure S54. HMBC (600 MHz, methanol- $\left.d_{4}\right)$ spectrum of 2-Hydroxymethylwaltherione C (8).

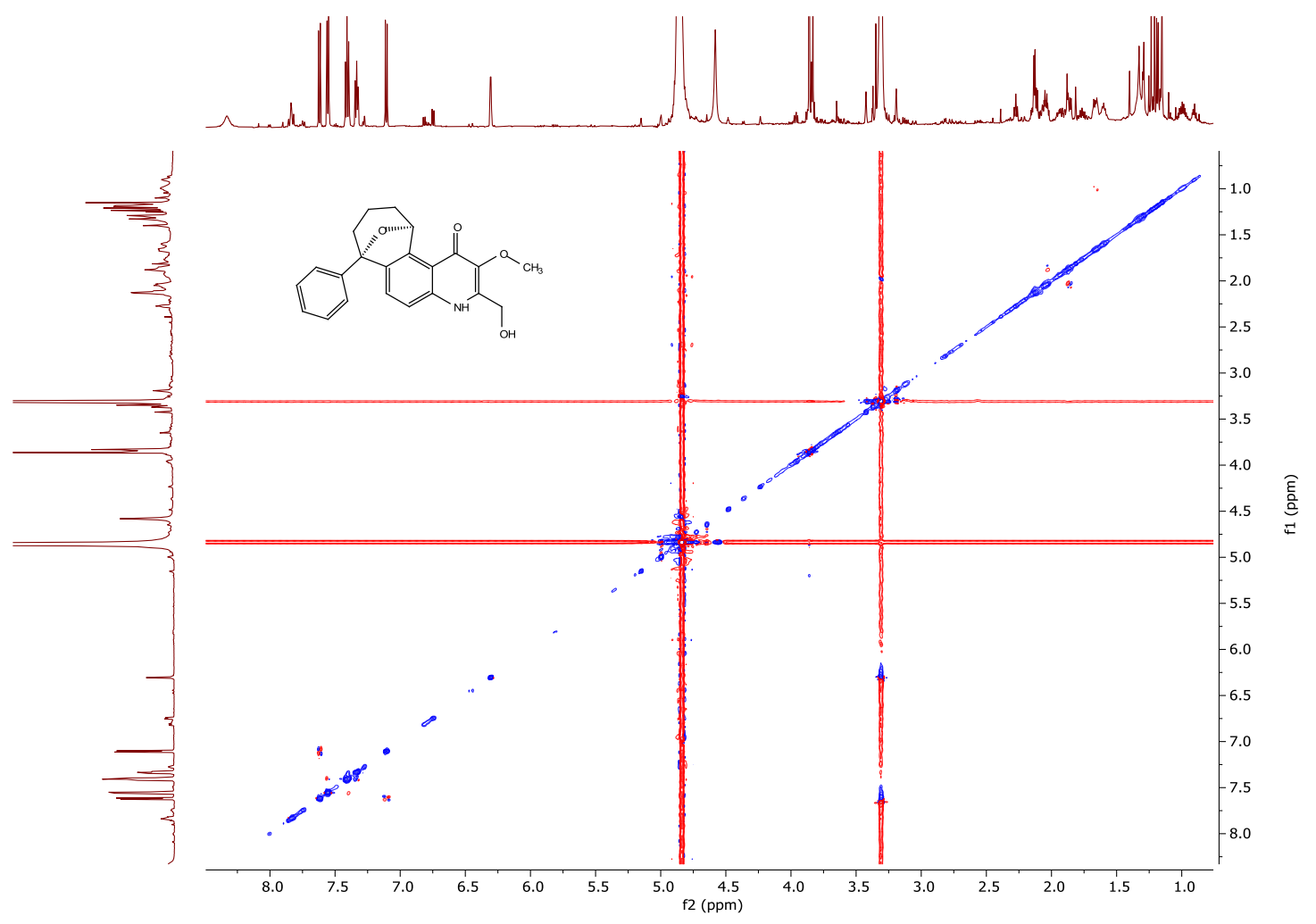

Figure S55. ROESY (600 MHz, methanol- $d_{4}$ ) spectrum of 2-Hydroxymethylwaltherione C (8). 


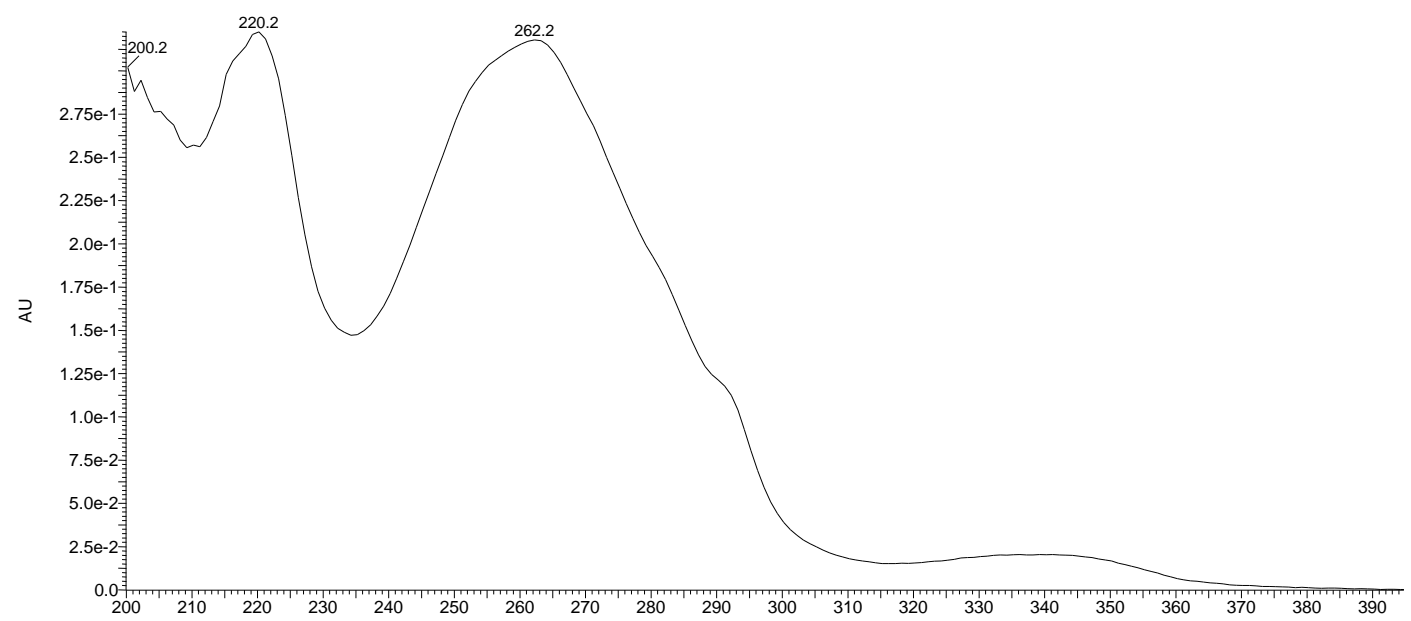

Figure S56. UV spectrum of 10-Hydroxymethylwaltherione C (9).
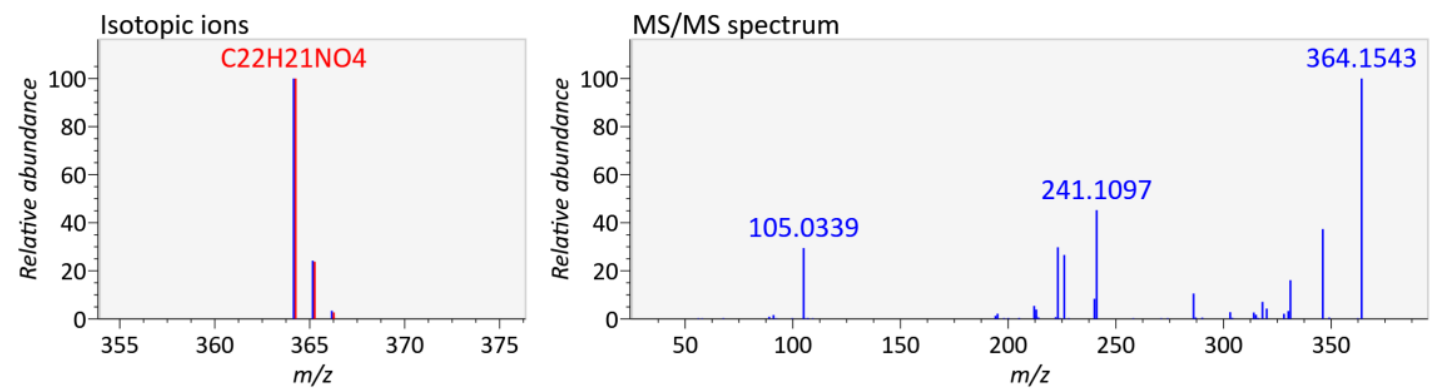

Figure S57. HRESIMS spectrum and MS/MS fragmentation of 10-Hydroxymethylwaltherione C (9) (Q Exactive Focus Hybrid quadripole-orbitrap mass spectrometer, positive mode). 


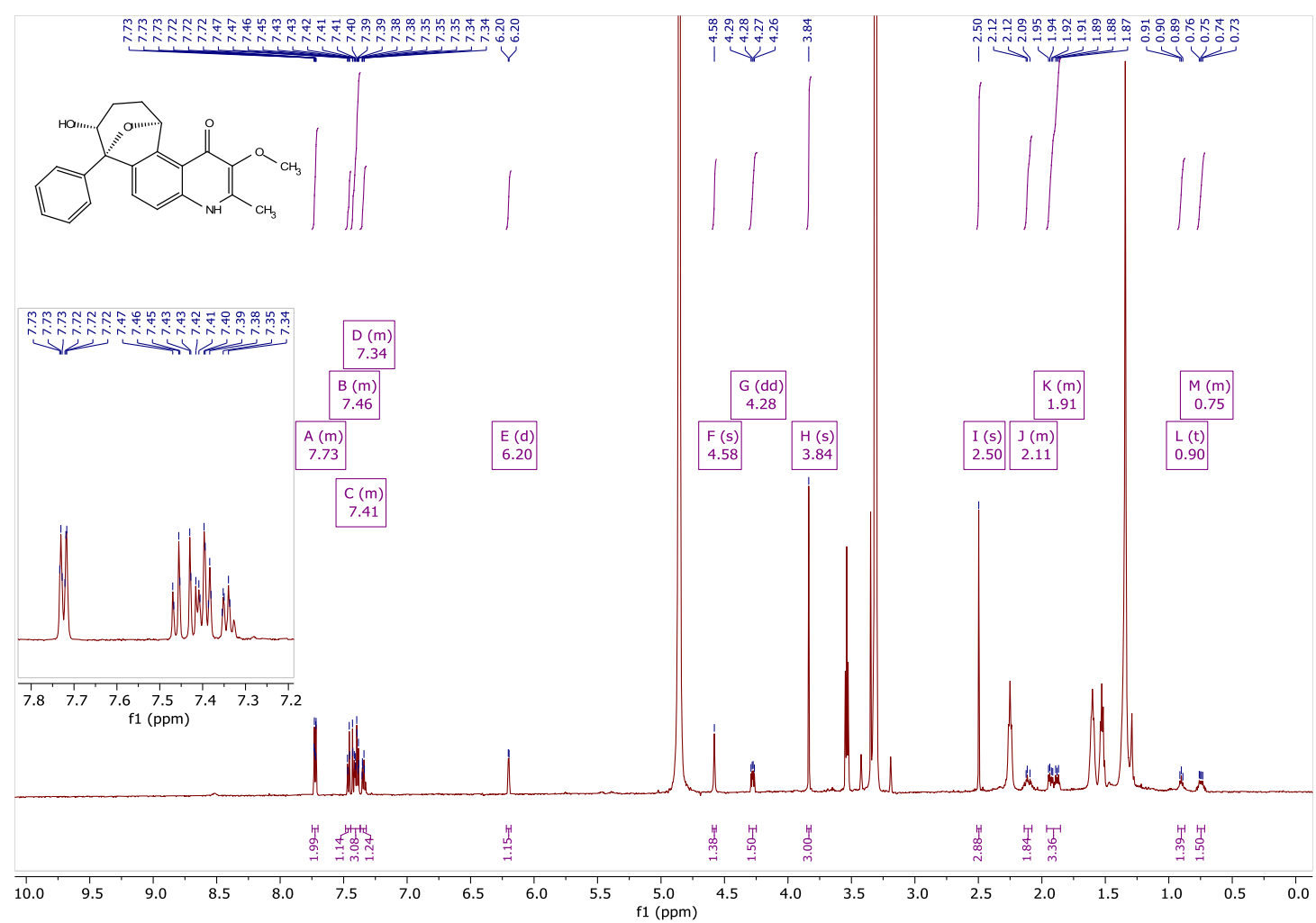

Figure S58. ${ }^{1} \mathrm{H}$ NMR (600 MHz, methanol- $\left.d_{4}\right)$ spectrum of 10-Hydroxymethylwaltherione C (9).

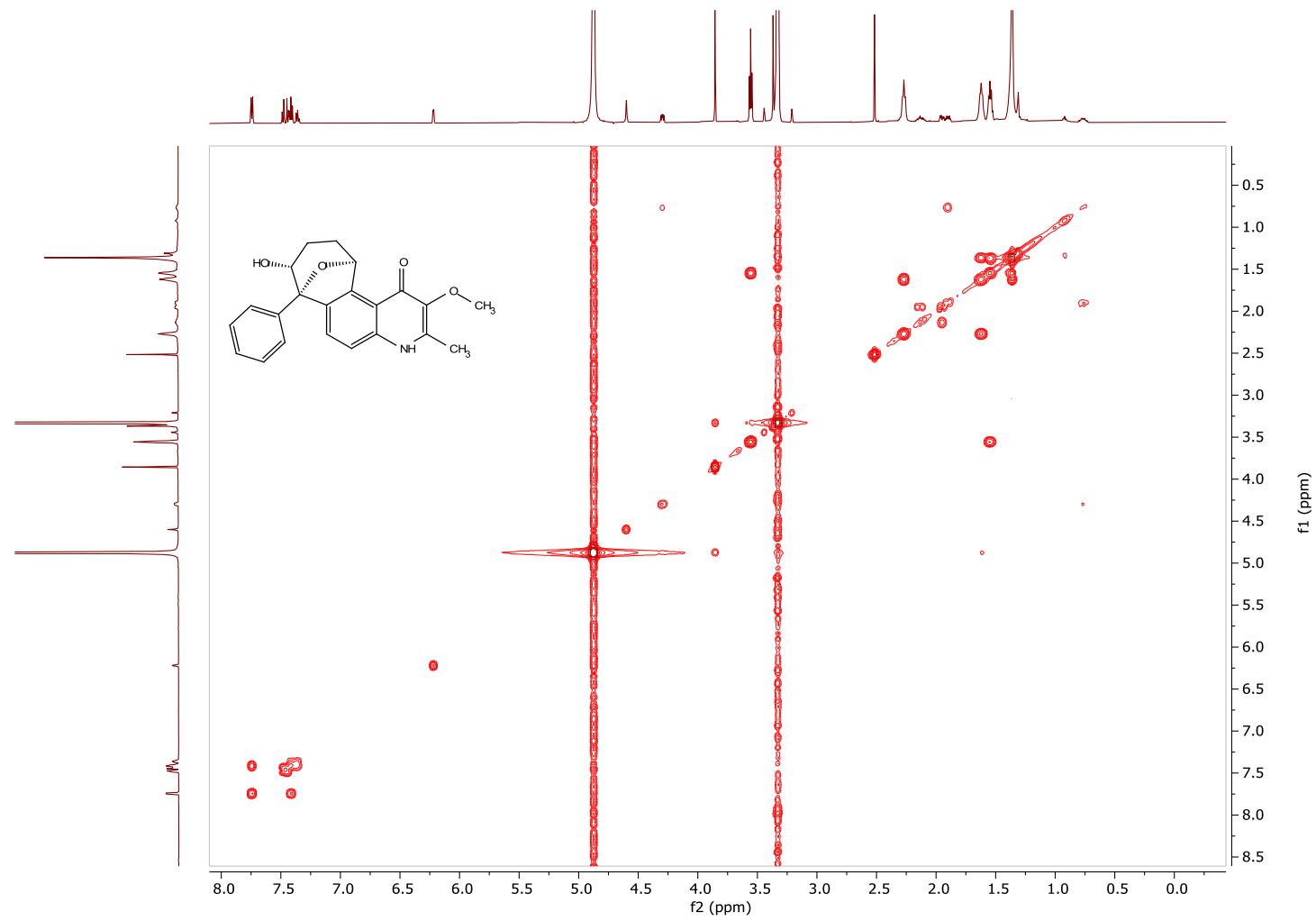

Figure S59. COSY (600 MHz, methanol- $\left.d_{4}\right)$ spectrum of 10-Hydroxymethylwaltherione C (9). 


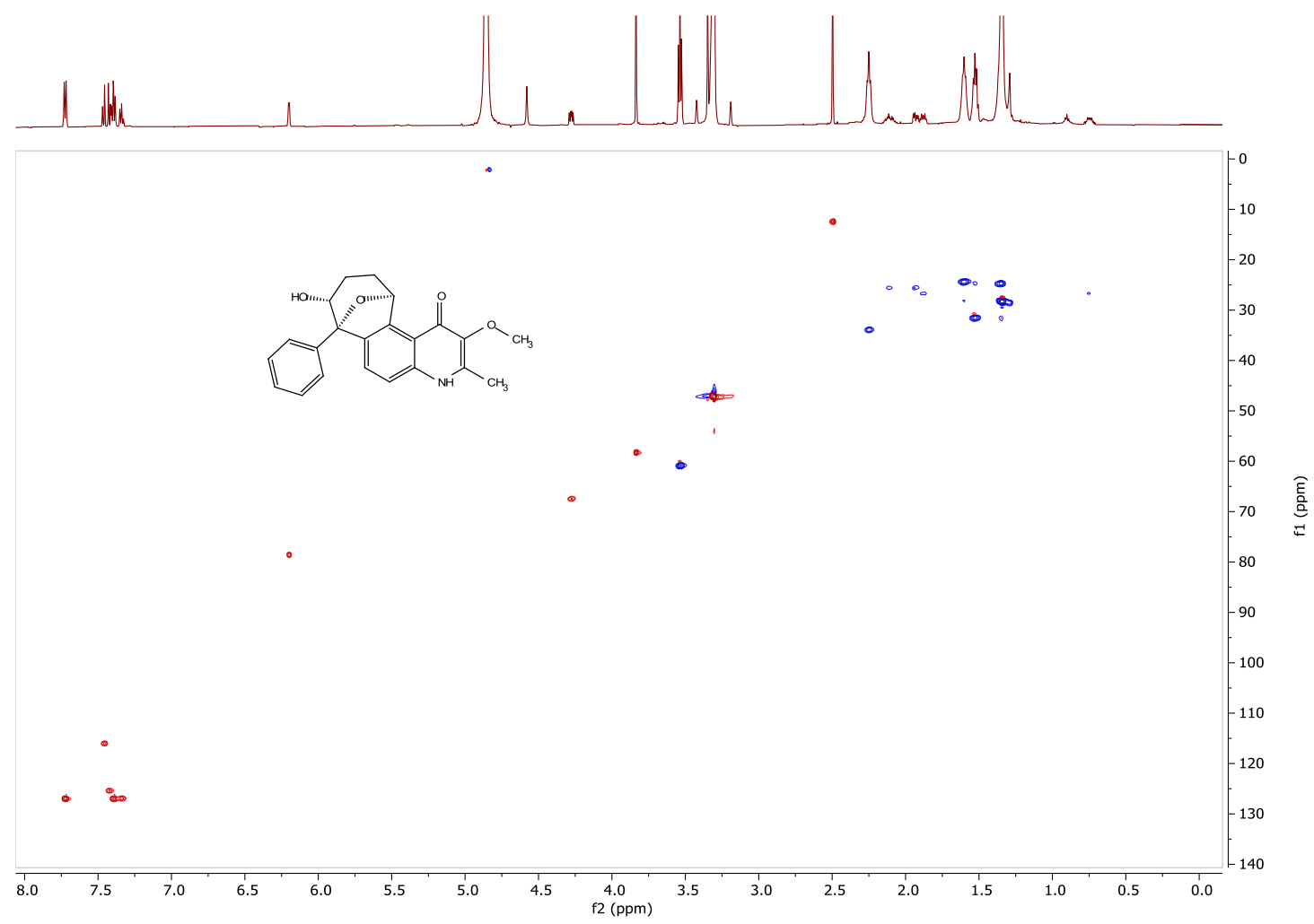

Figure S60. HSQC (600 MHz, methanol- $\left.d_{4}\right)$ spectrum of 10-Hydroxymethylwaltherione C (9).

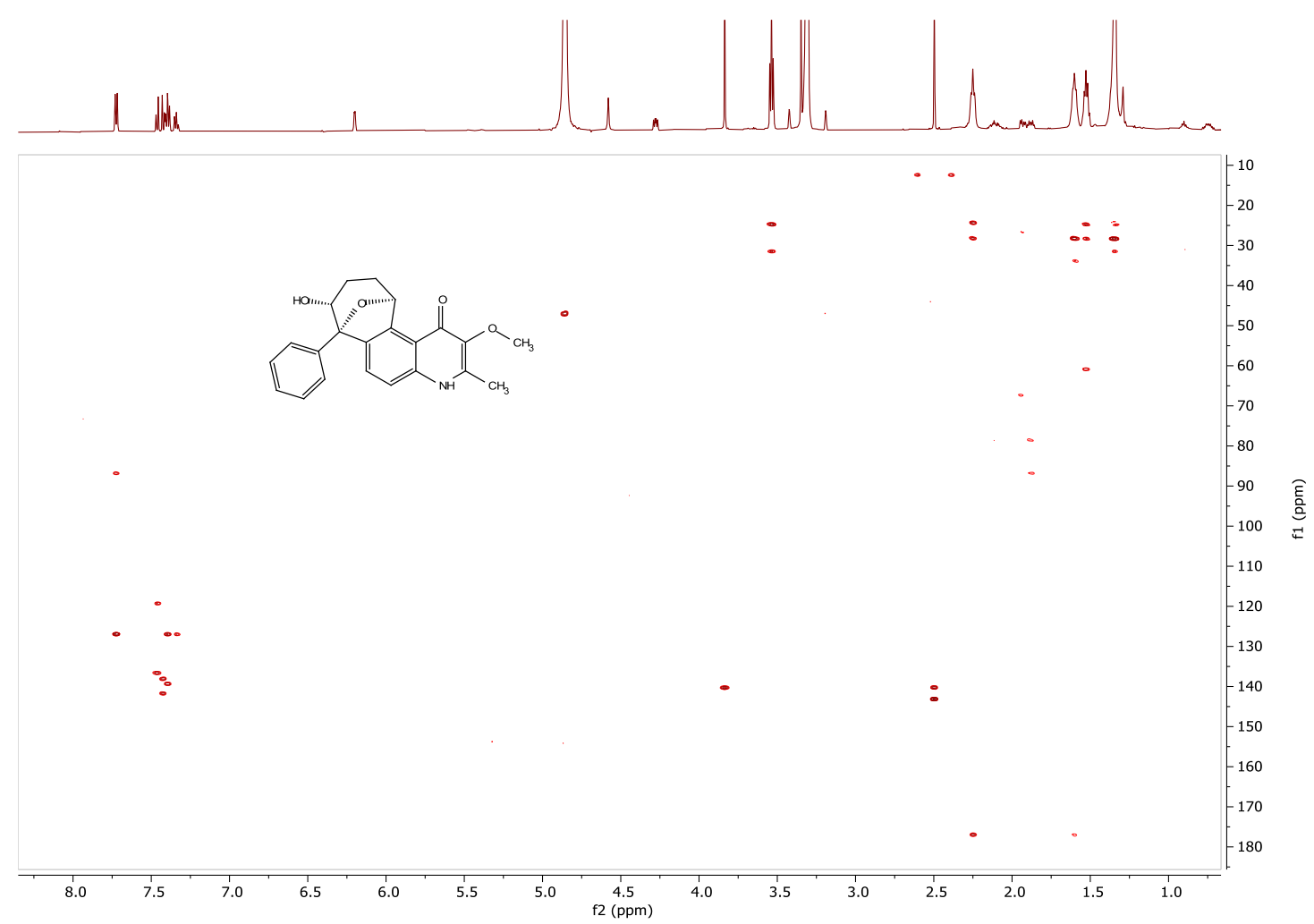

Figure S61. HMBC (600 MHz, methanol- $d_{4}$ ) spectrum of 10-Hydroxymethylwaltherione C (9). 


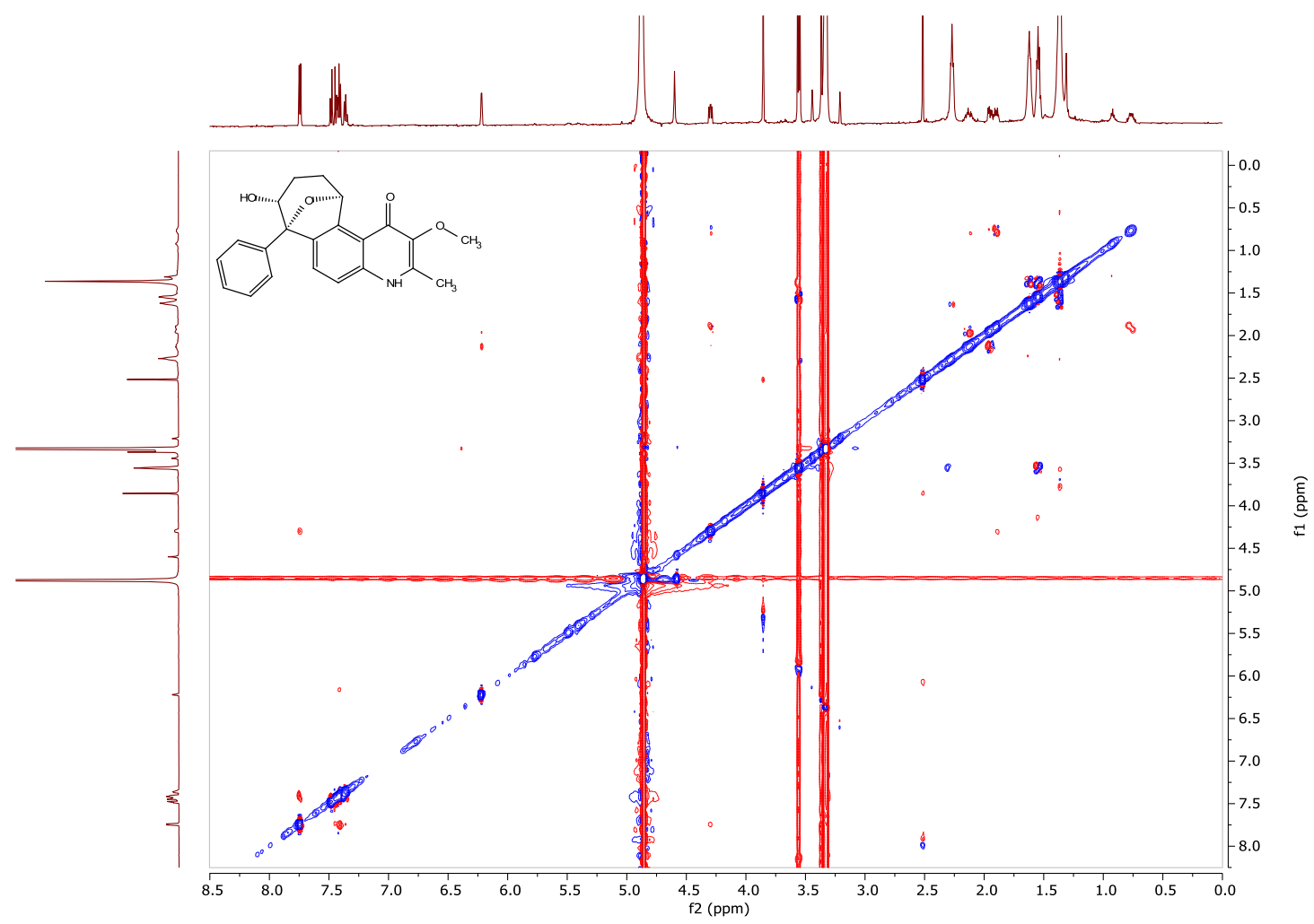

Figure S62. ROESY (600 MHz, methanol- $d_{4}$ ) spectrum of 10-Hydroxymethylwaltherione C (9).

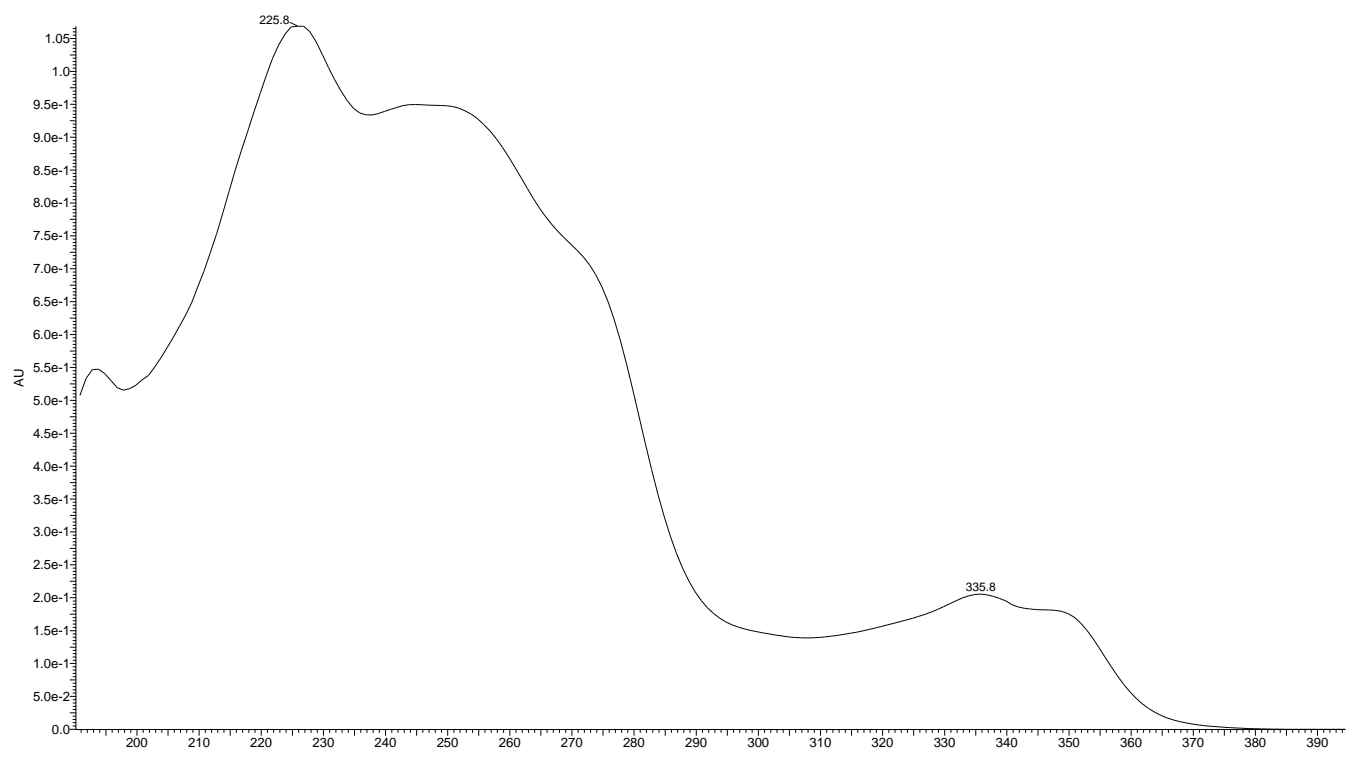

Figure S63. UV spectrum of Waltherione U (10). 

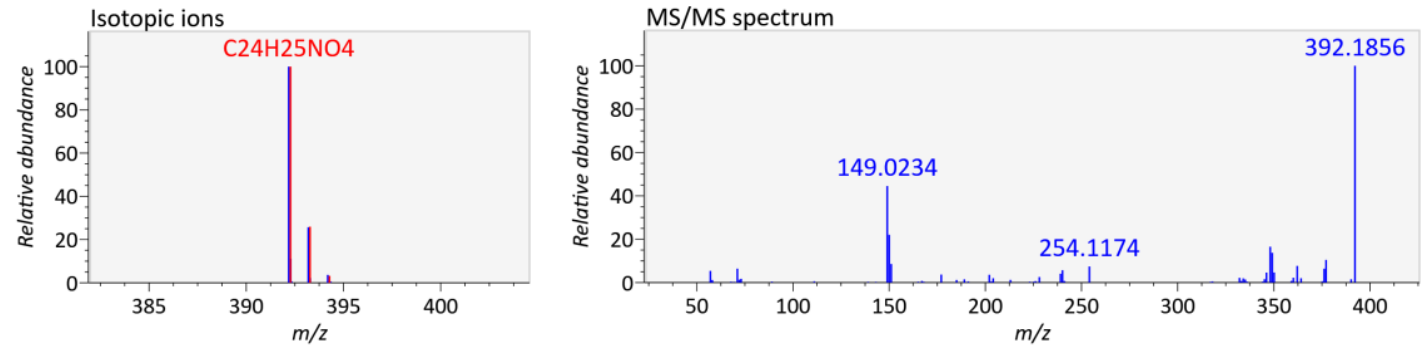

Figure S64. HRESIMS spectrum and MS/MS fragmentation of Waltherione U (10) (Q Exactive Focus Hybrid quadripole-orbitrap mass spectrometer, positive mode).

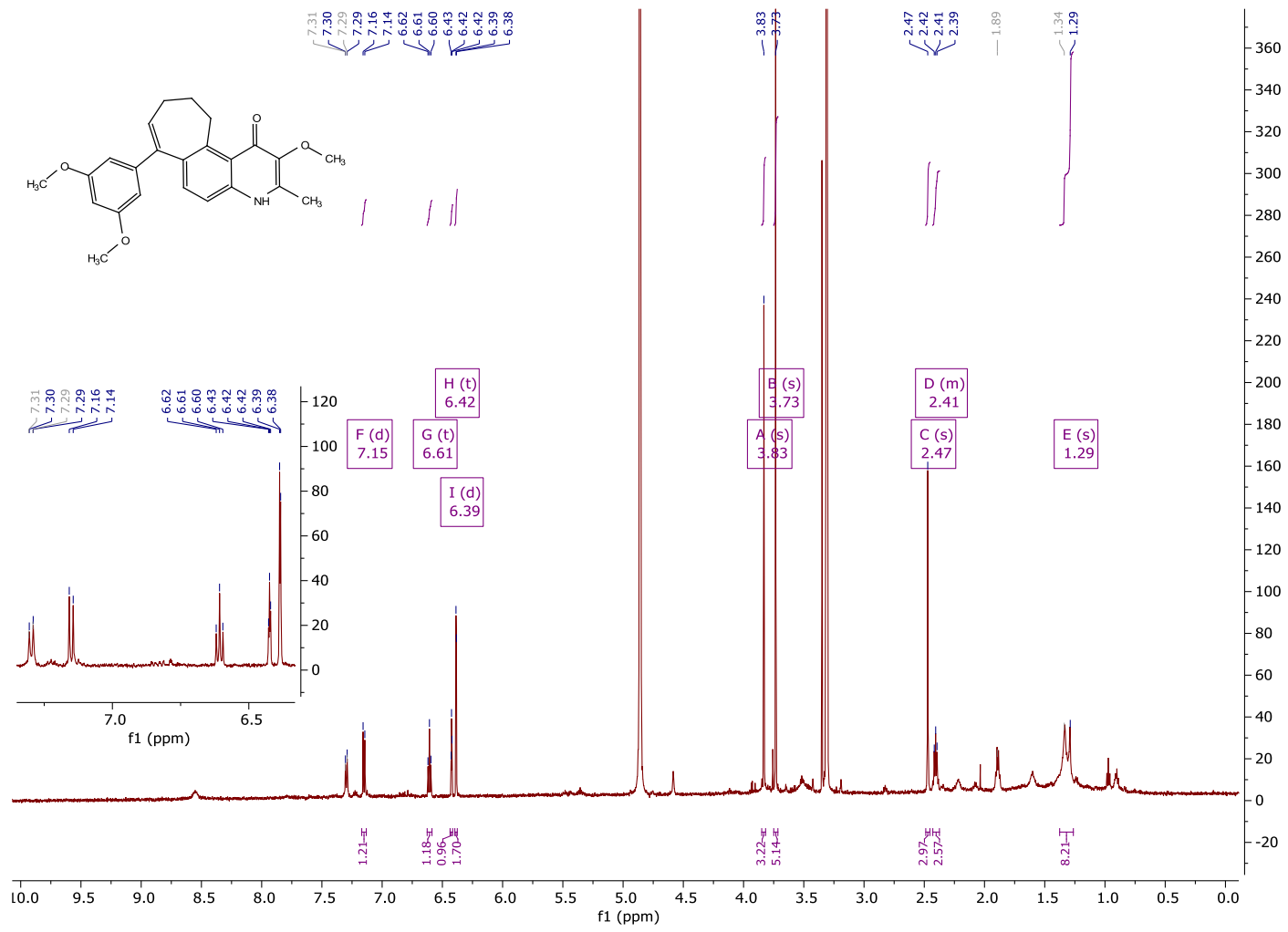

Figure S65. ${ }^{1} \mathrm{H}$ NMR $\left(600 \mathrm{MHz}\right.$, methanol- $\left.d_{4}\right)$ spectrum of Waltherione U (10). 


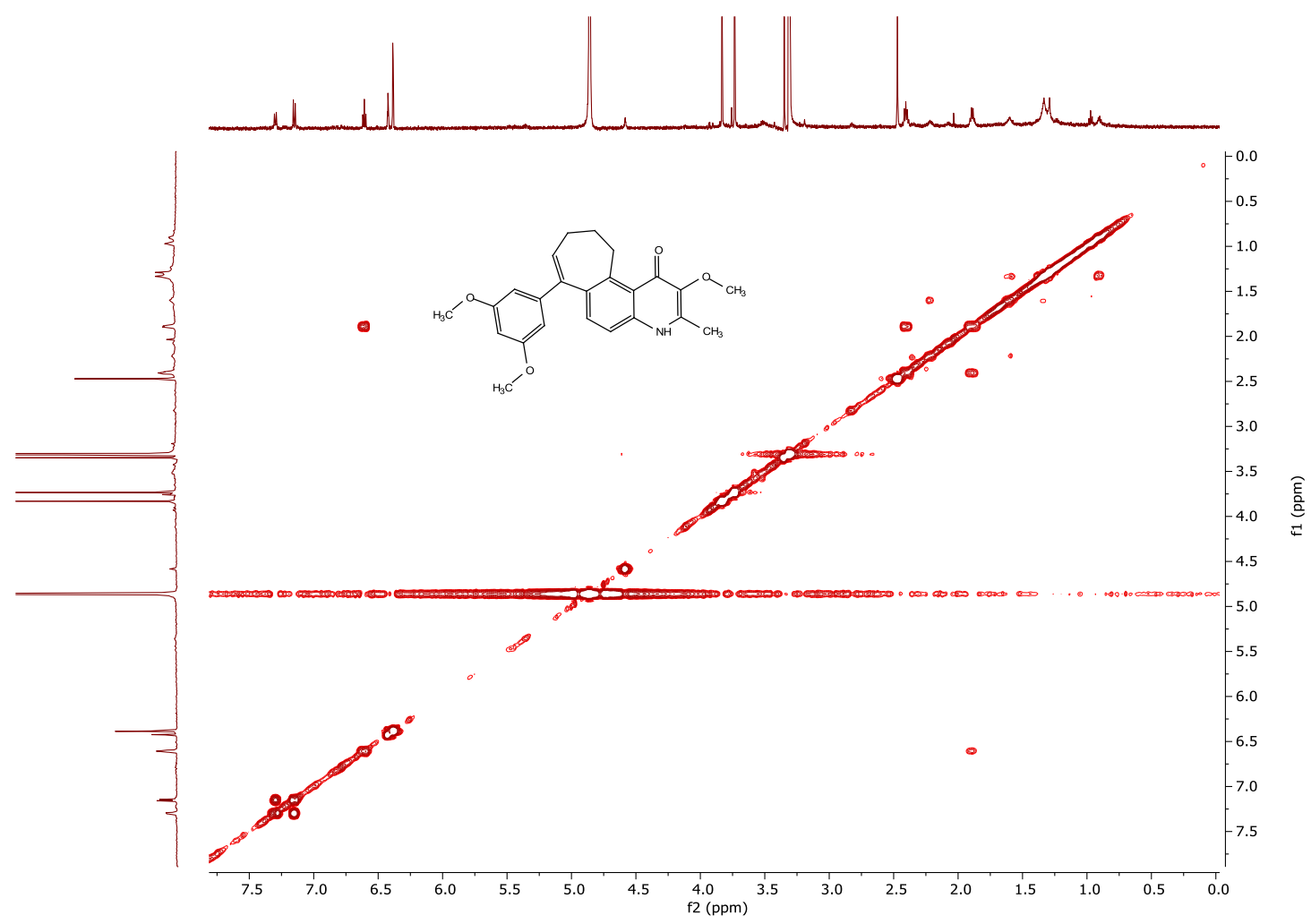

Figure S66. COSY (600 MHz, methanol- $\left.d_{4}\right)$ spectrum of Waltherione U (10).

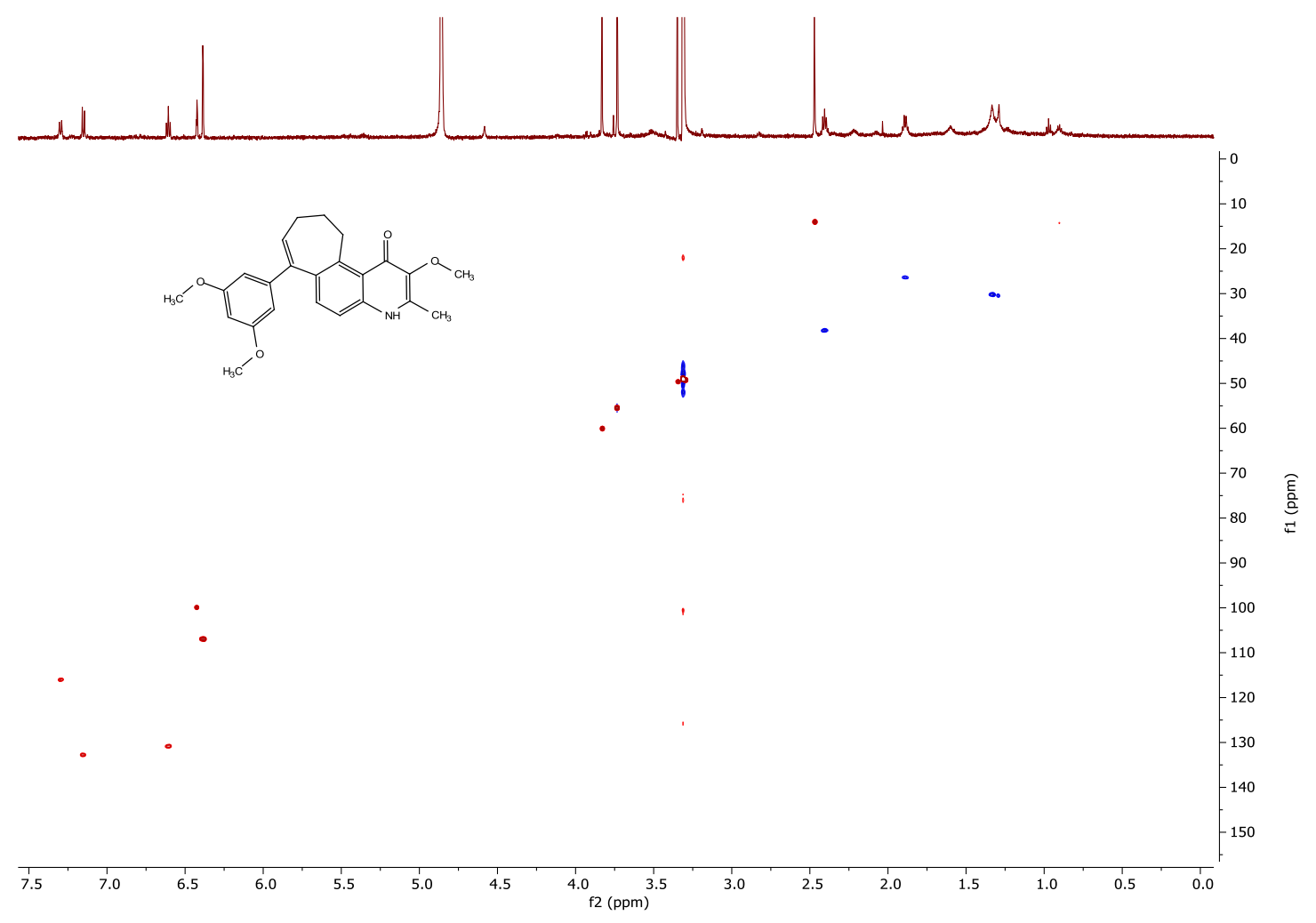

Figure S67. HSQC (600 MHz, methanol- $d_{4}$ ) spectrum of Waltherione U (10). 


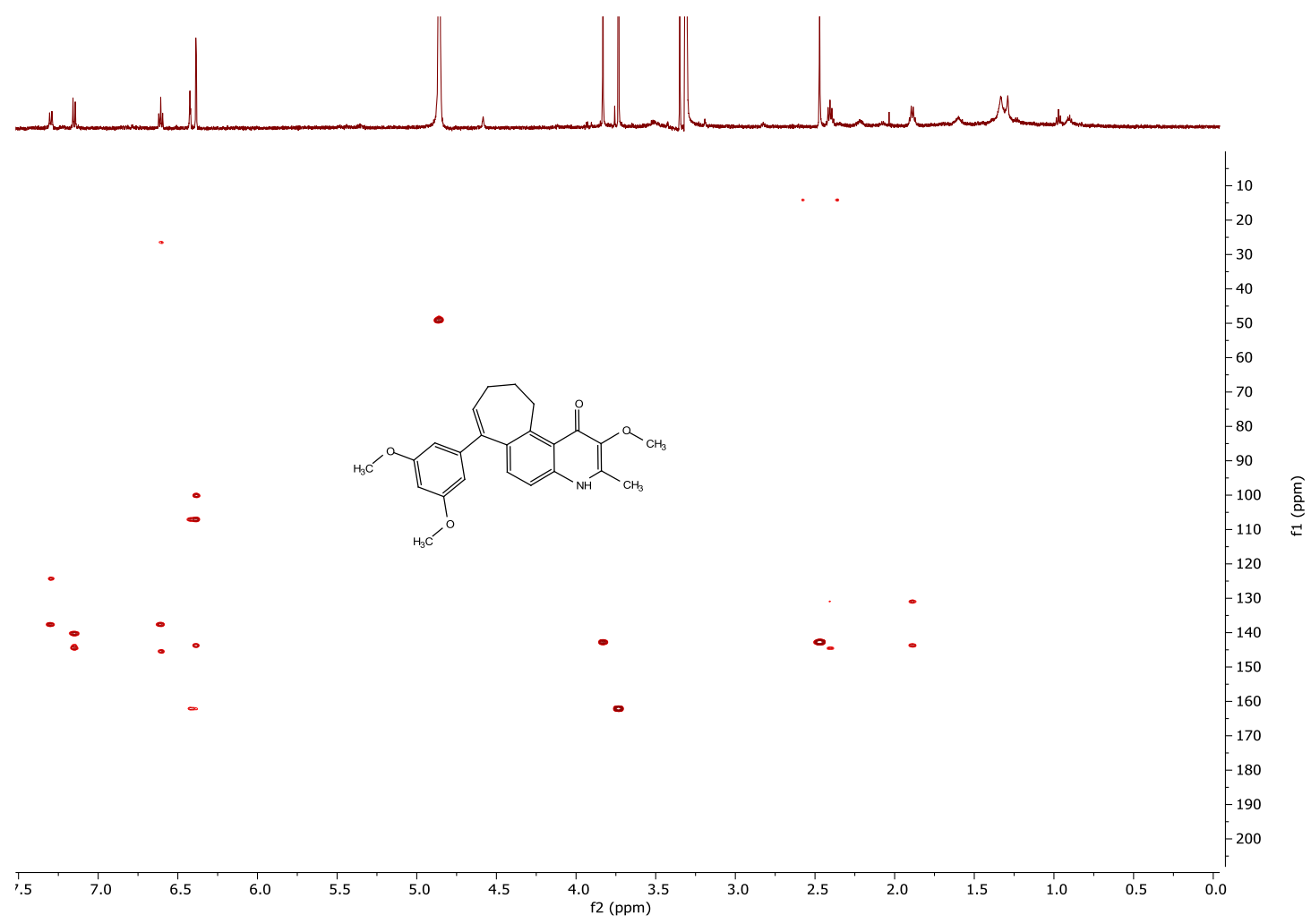

Figure S68. HMBC (600 MHz, methanol- $\left.d_{4}\right)$ spectrum of Waltherione U (10).

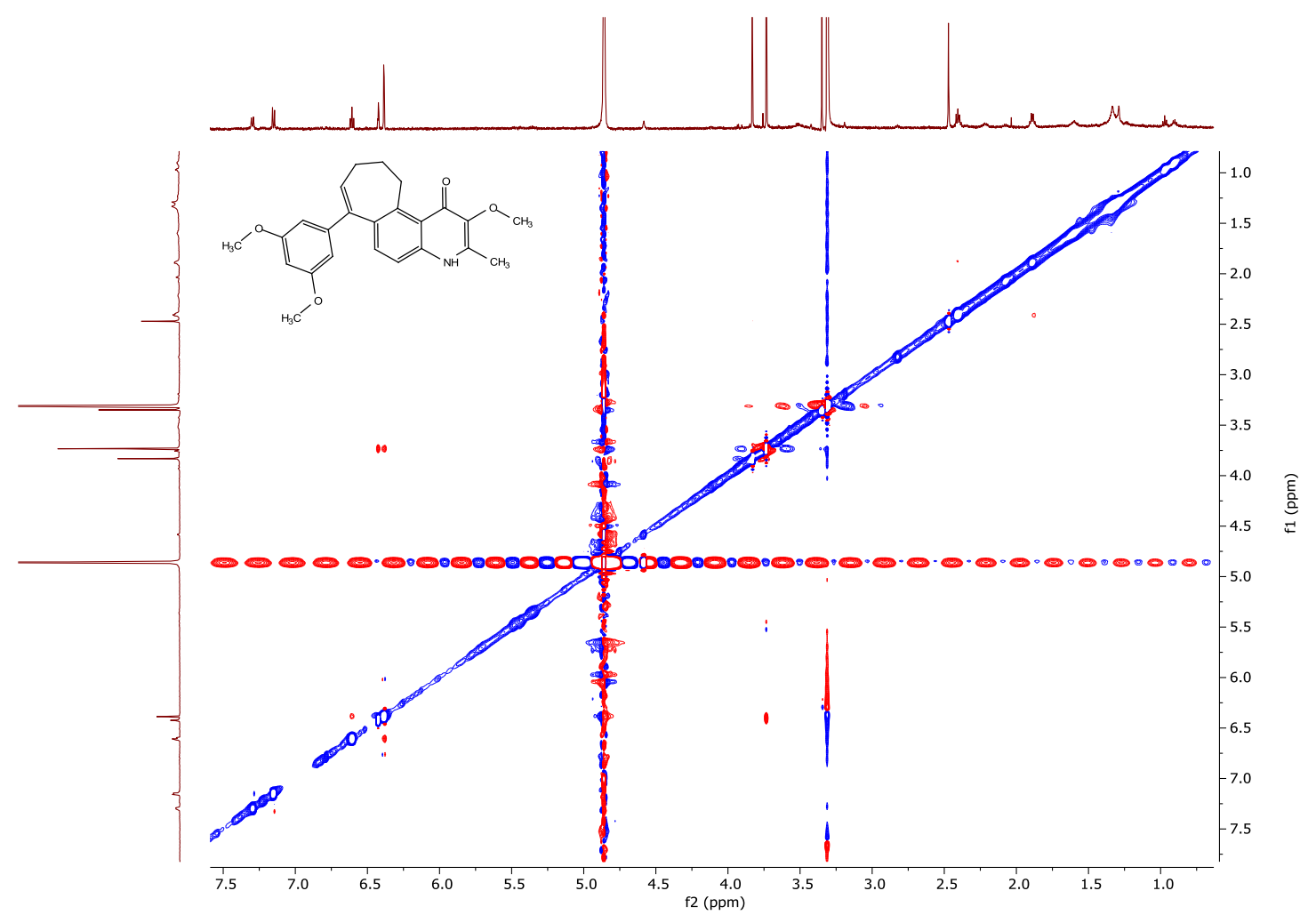

Figure S69. ROESY (600 MHz, methanol- $\left.d_{4}\right)$ spectrum of Waltherione U (10). 


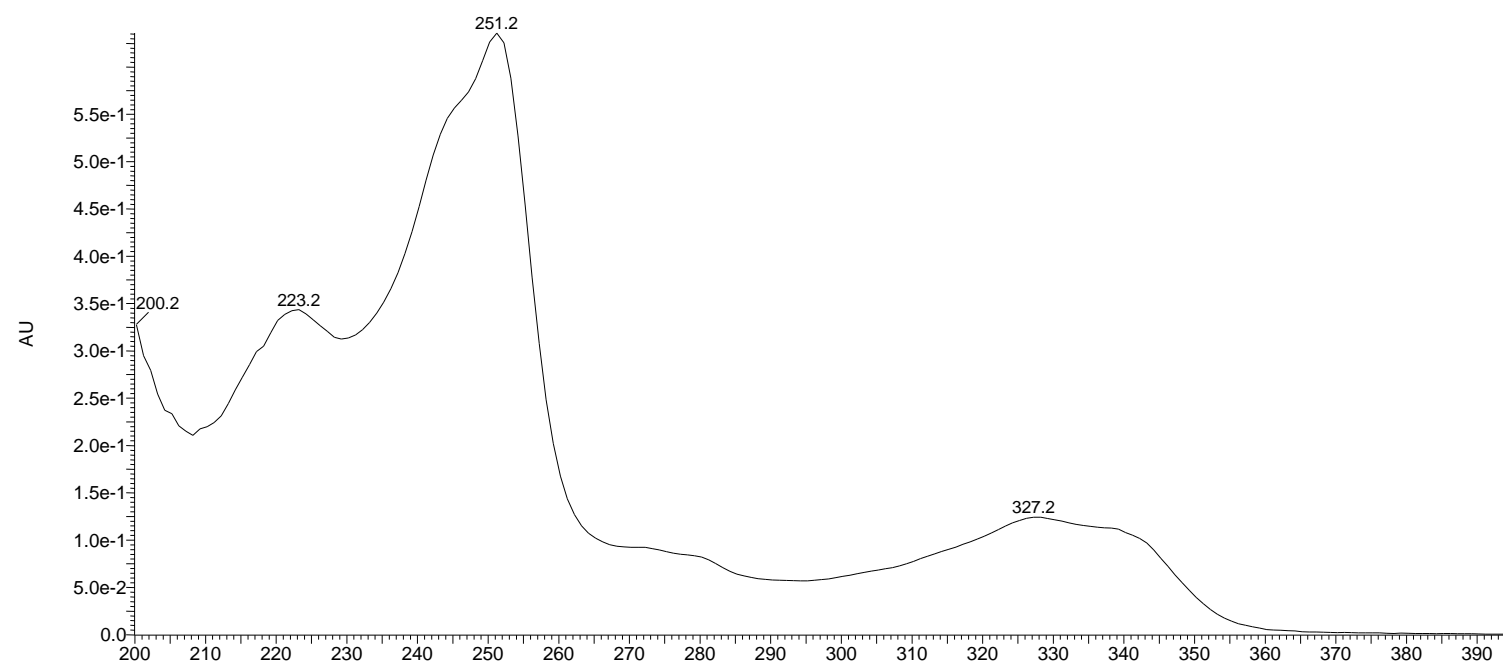

Figure S70. UV spectrum of Waltherione V (11).
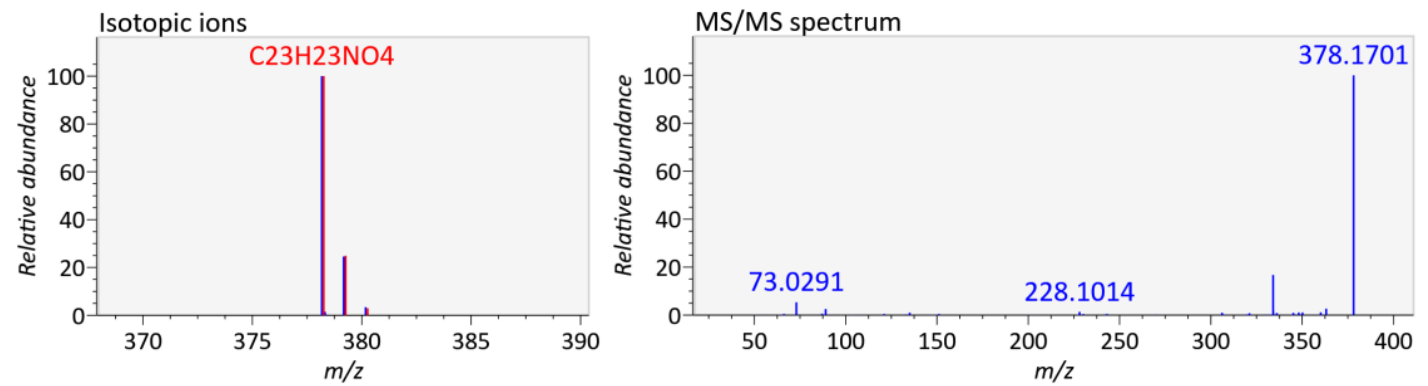

Figure S71. HRESIMS spectrum and MS/MS fragmentation of Waltherione V (11) (Q Exactive Focus Hybrid quadripole-orbitrap mass spectrometer, positive mode). 


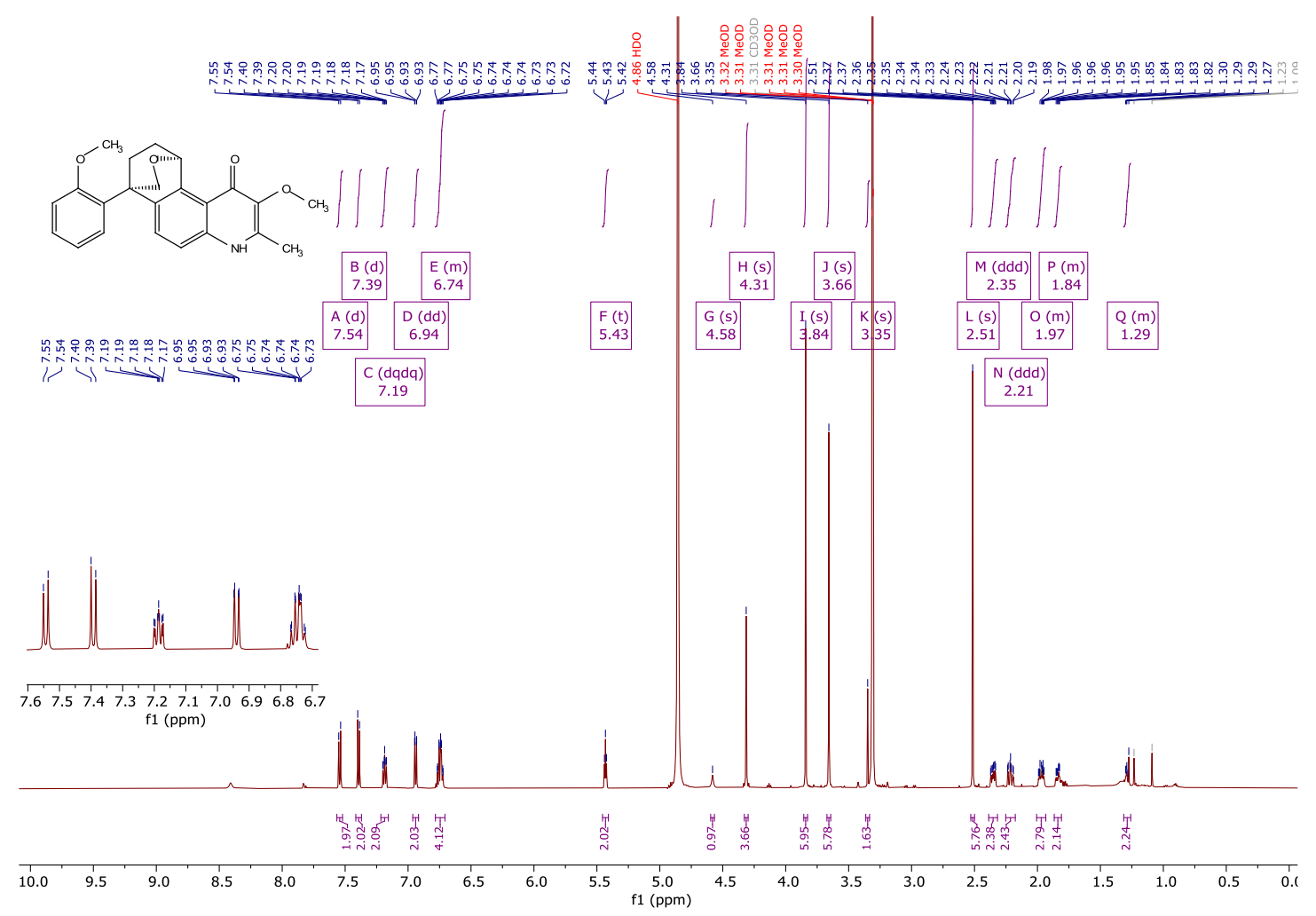

Figure S72. ${ }^{1} \mathrm{H}$ NMR (600 MHz, methanol- $\left.d_{4}\right)$ spectrum of Waltherione V (11).

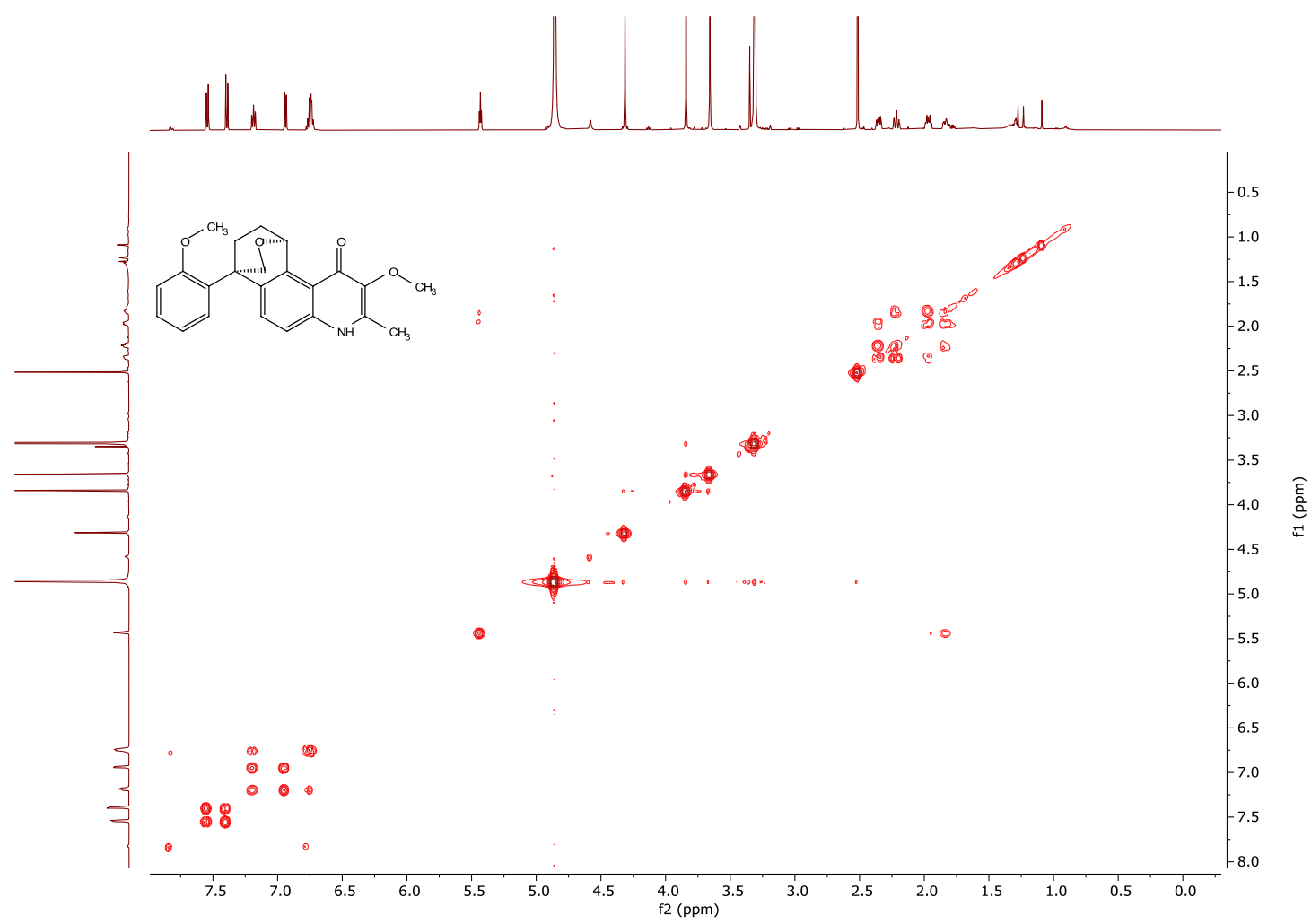

Figure S73. COSY (600 MHz, methanol- $d_{4}$ ) spectrum of Waltherione V (11). 


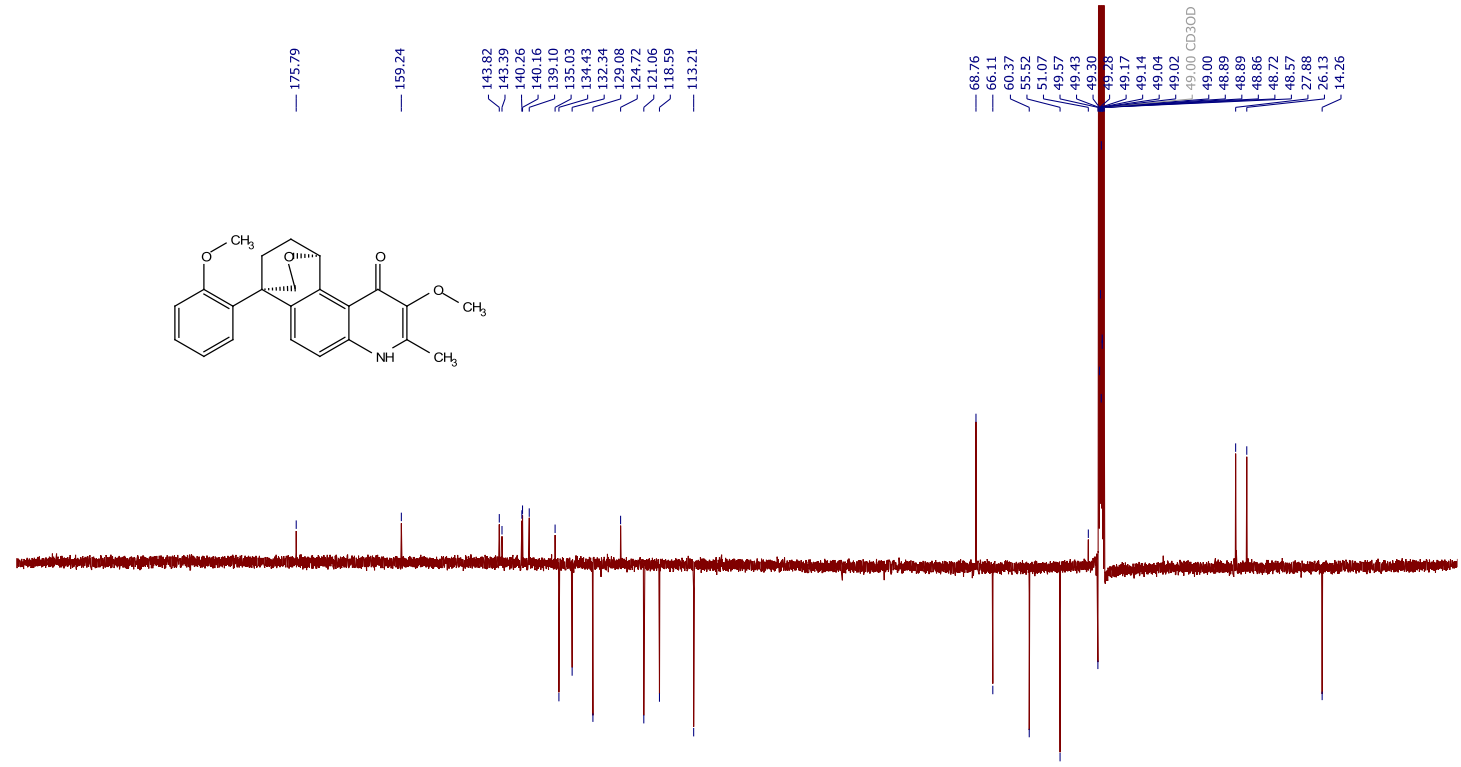

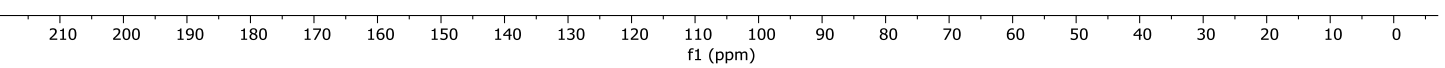

Figure S74. DEPTQ (150 MHz, methanol- $\left.d_{4}\right)$ spectrum of Waltherione V (11).

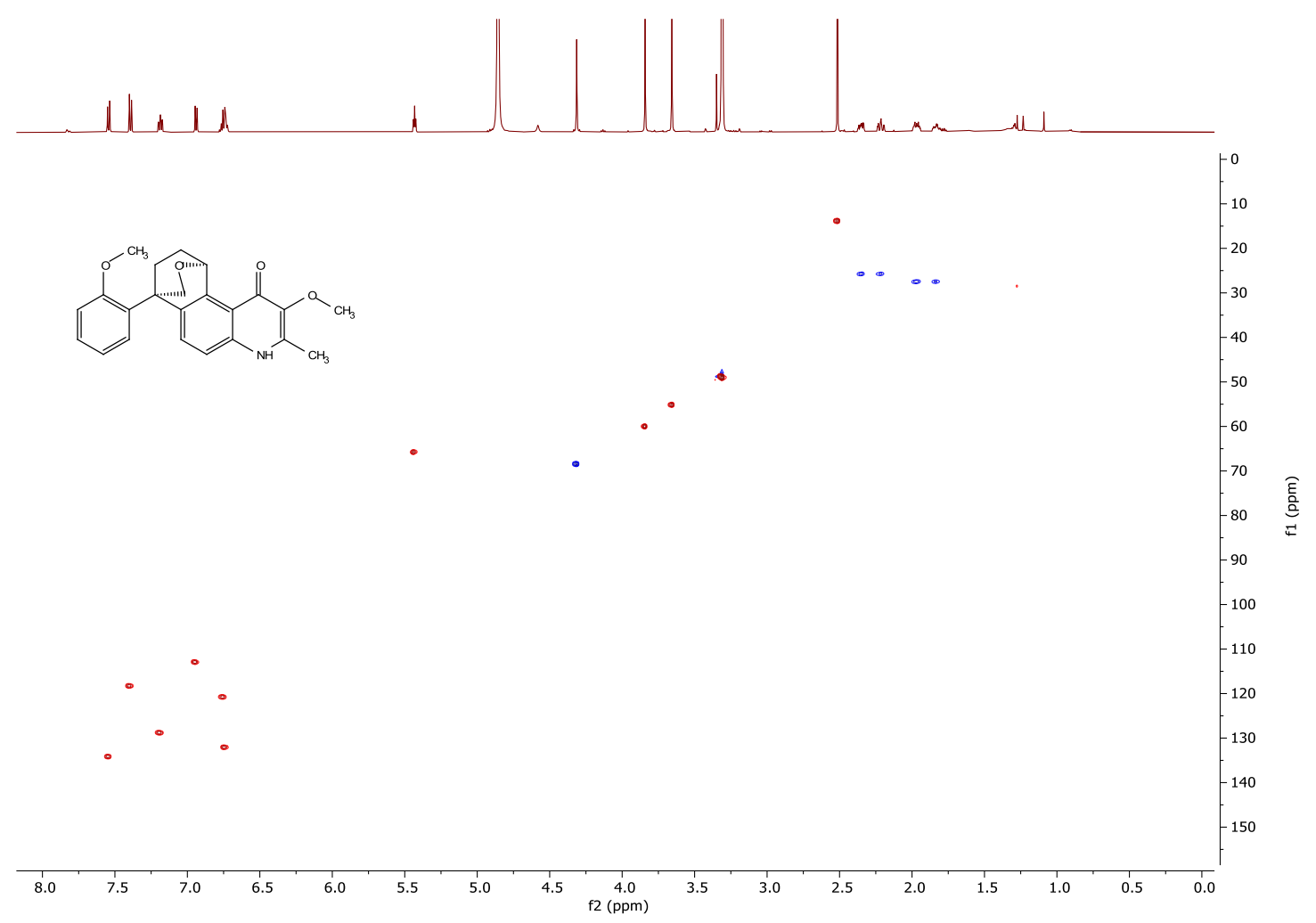

Figure S75. HSQC (600 MHz, methanol- $\left.d_{4}\right)$ spectrum of Waltherione V (11). 


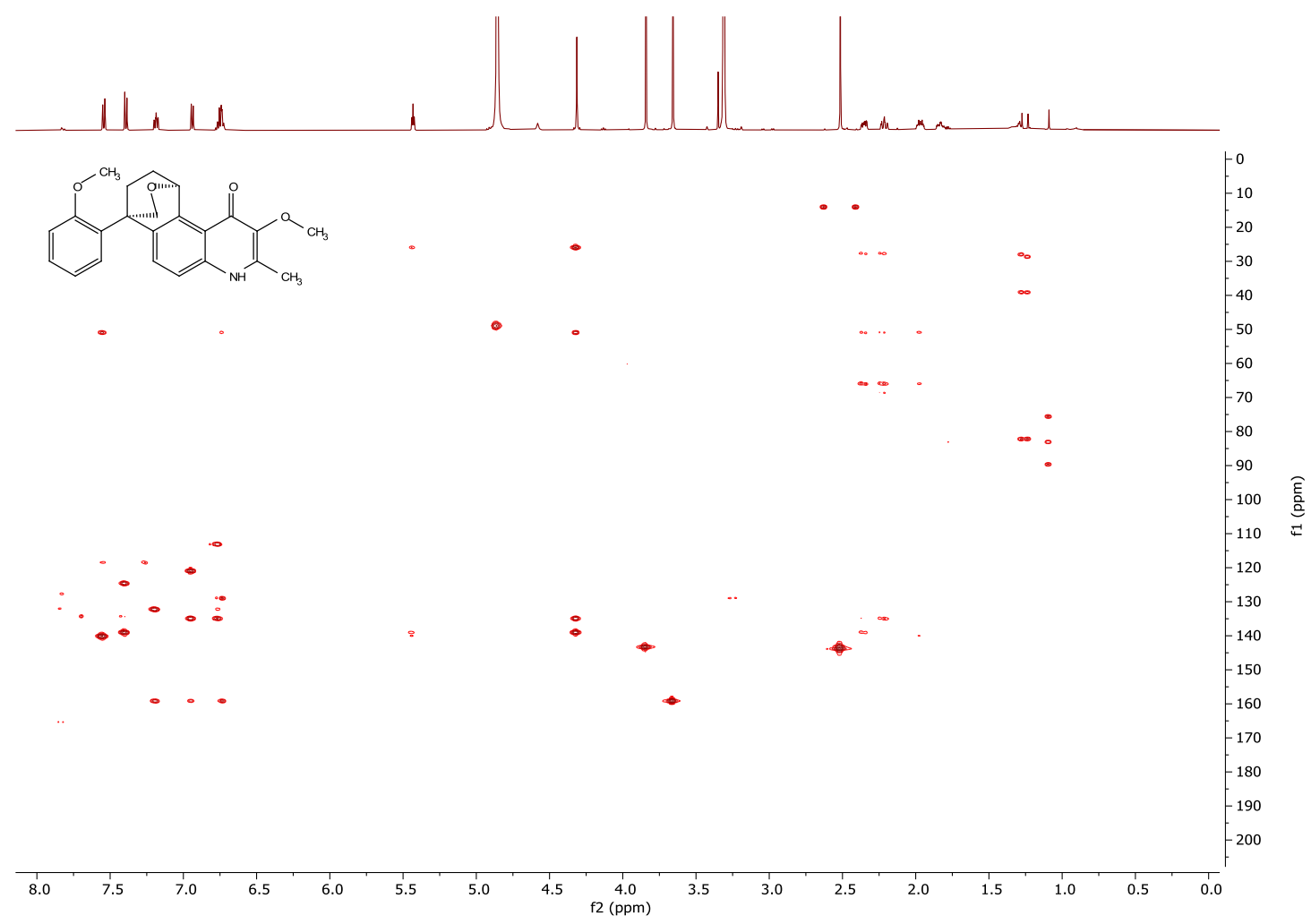

Figure S76. HMBC (600 MHz, methanol- $\left.d_{4}\right)$ spectrum of Waltherione V (11).

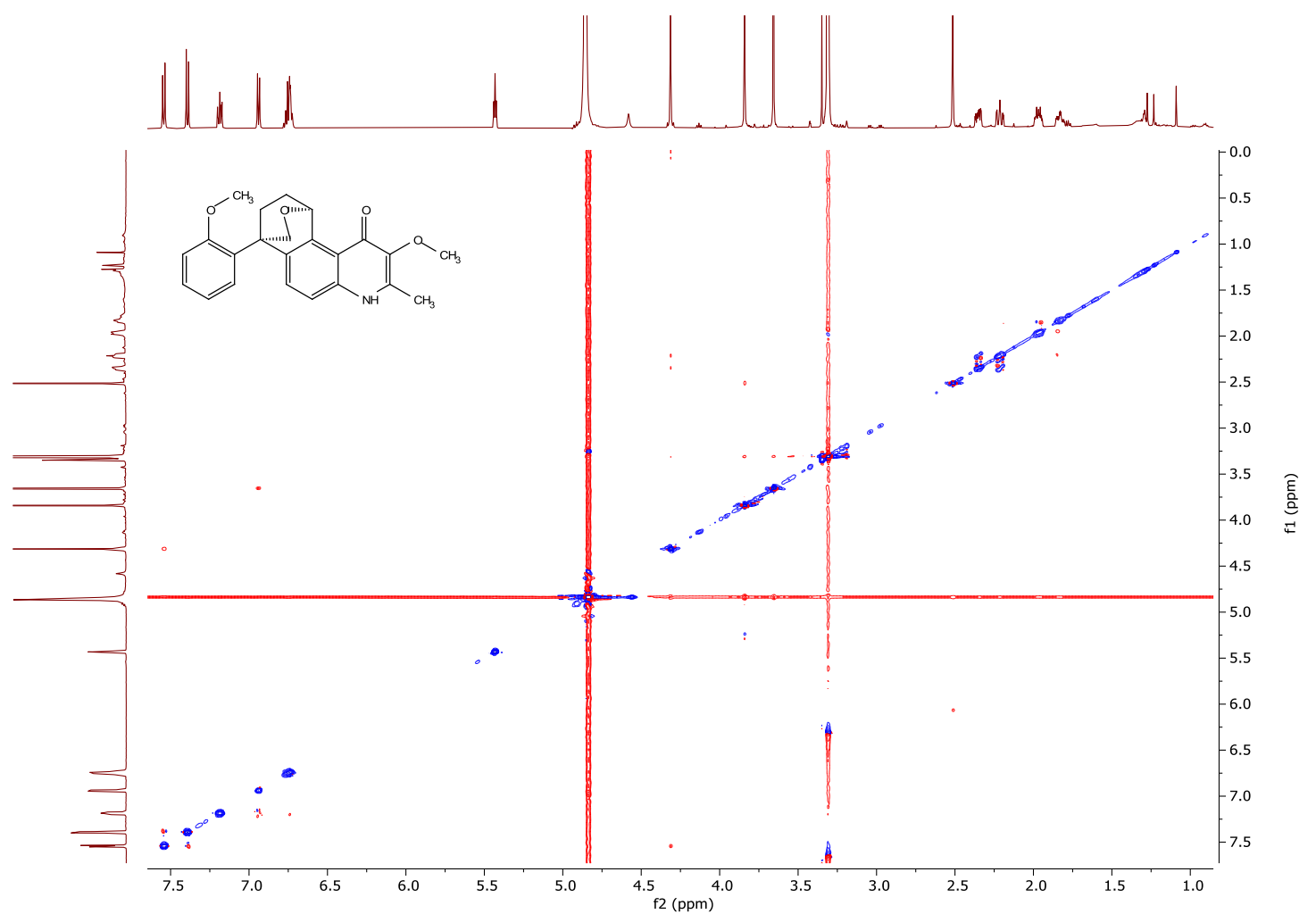

Figure S77. ROESY (600 MHz, methanol- $\left.d_{4}\right)$ spectrum of Waltherione V (11). 


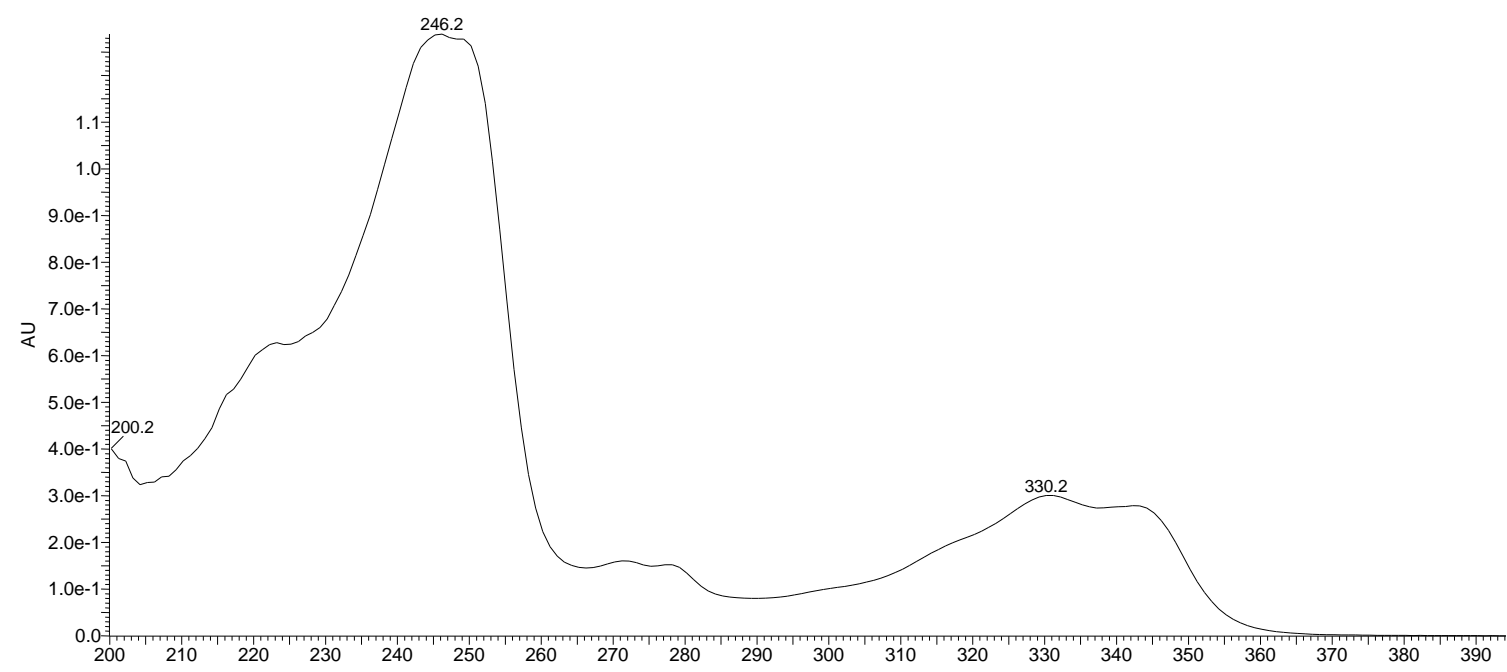

Figure S78. UV spectrum of 13-Methoxywaltherione V (12).
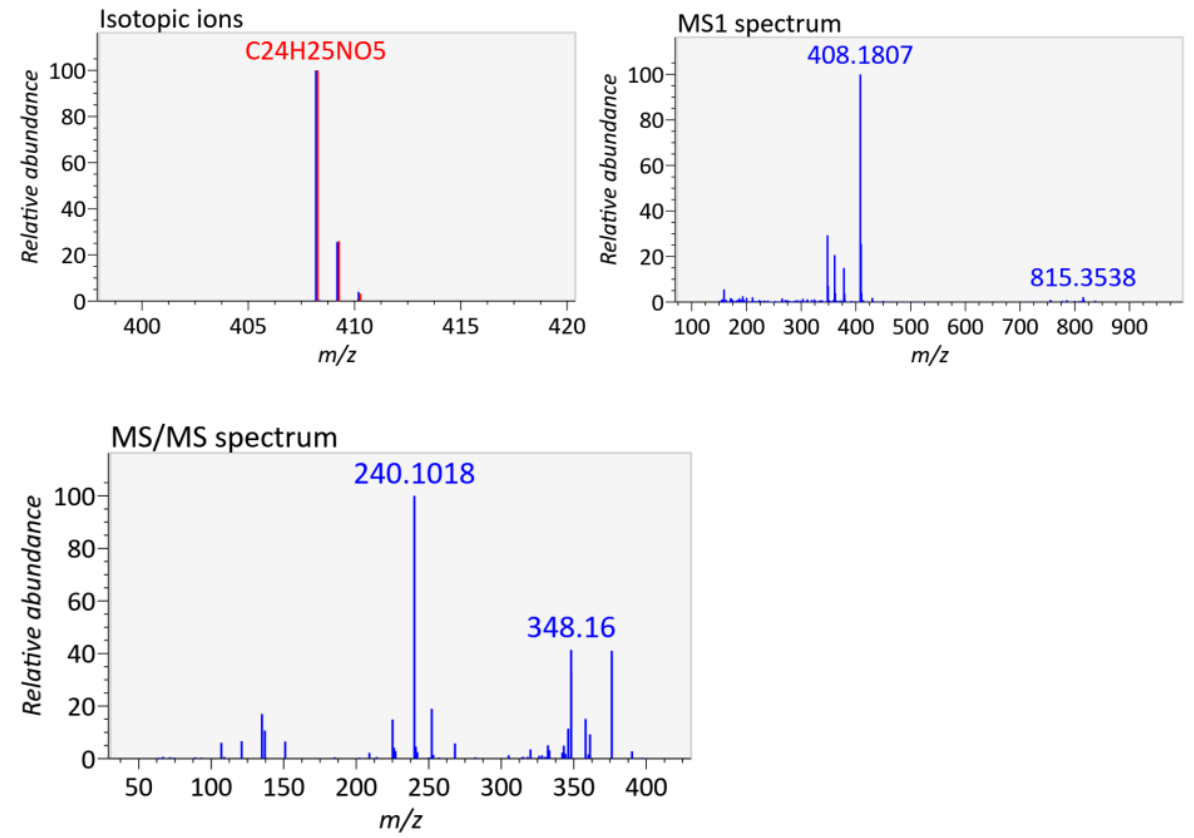

Figure S79. HRESIMS spectrum and MS/MS fragmentation of 13-Methoxywaltherione V (12) (Q Exactive Focus Hybrid quadripole-orbitrap mass spectrometer, positive mode). 


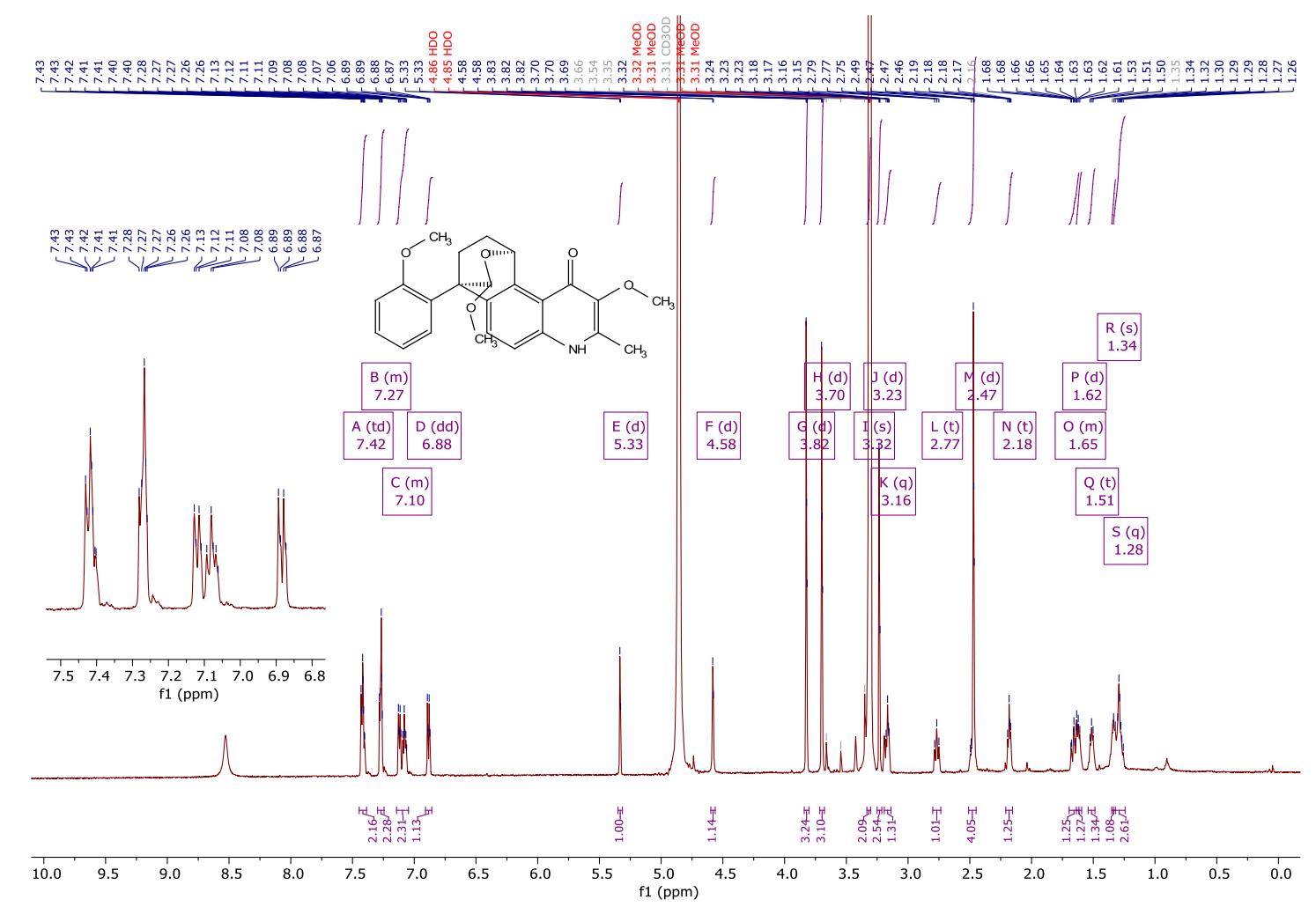

Figure S80. ${ }^{1} \mathrm{H}$ NMR (600 MHz, methanol- $d_{4}$ ) spectrum of 13-Methoxywaltherione V (12).

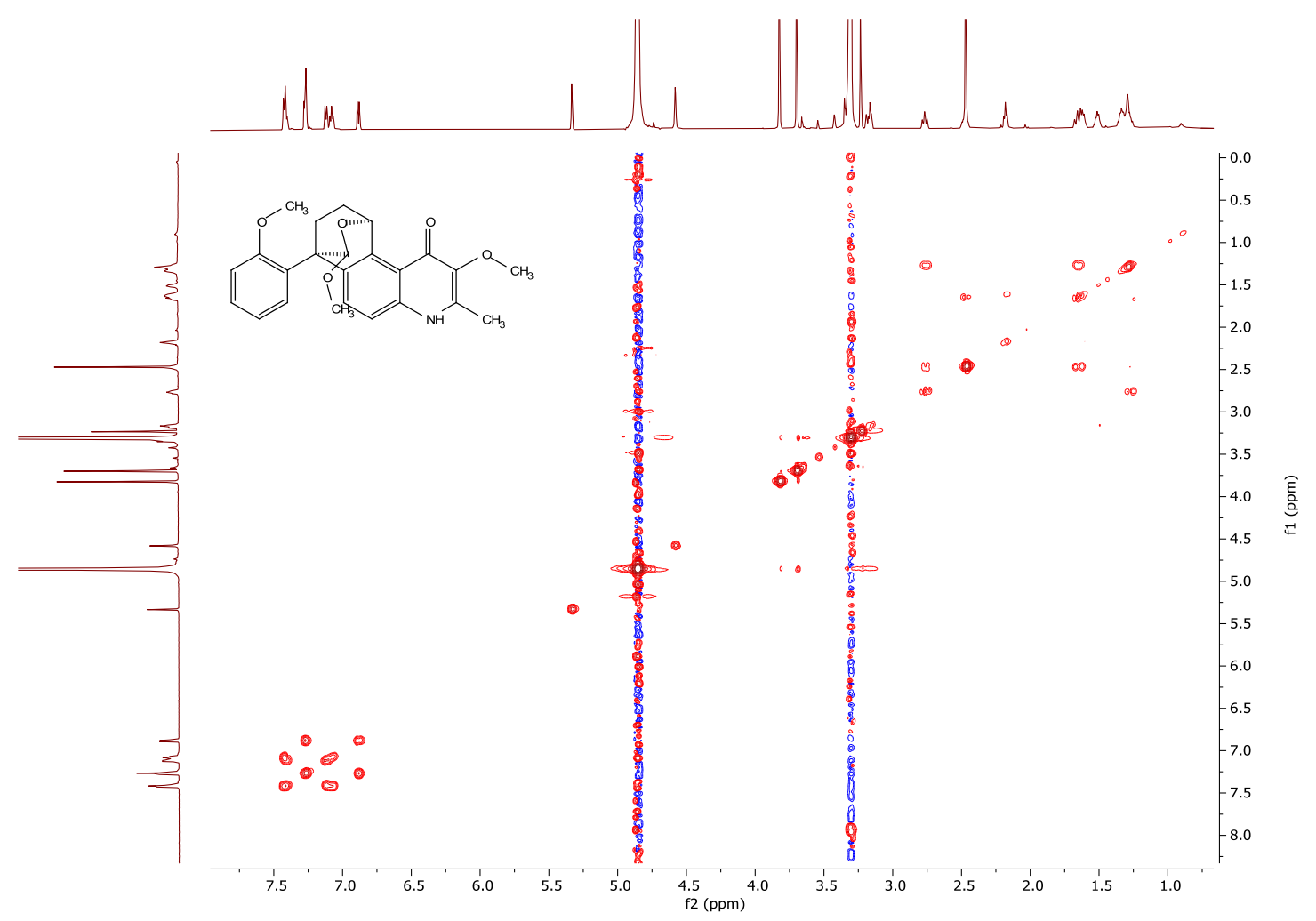

Figure S81. COSY (600 MHz, methanol- $\left.d_{4}\right)$ spectrum of 13-Methoxywaltherione V (12). 


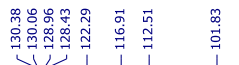

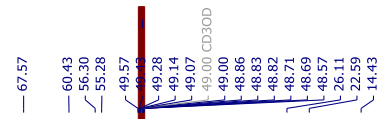
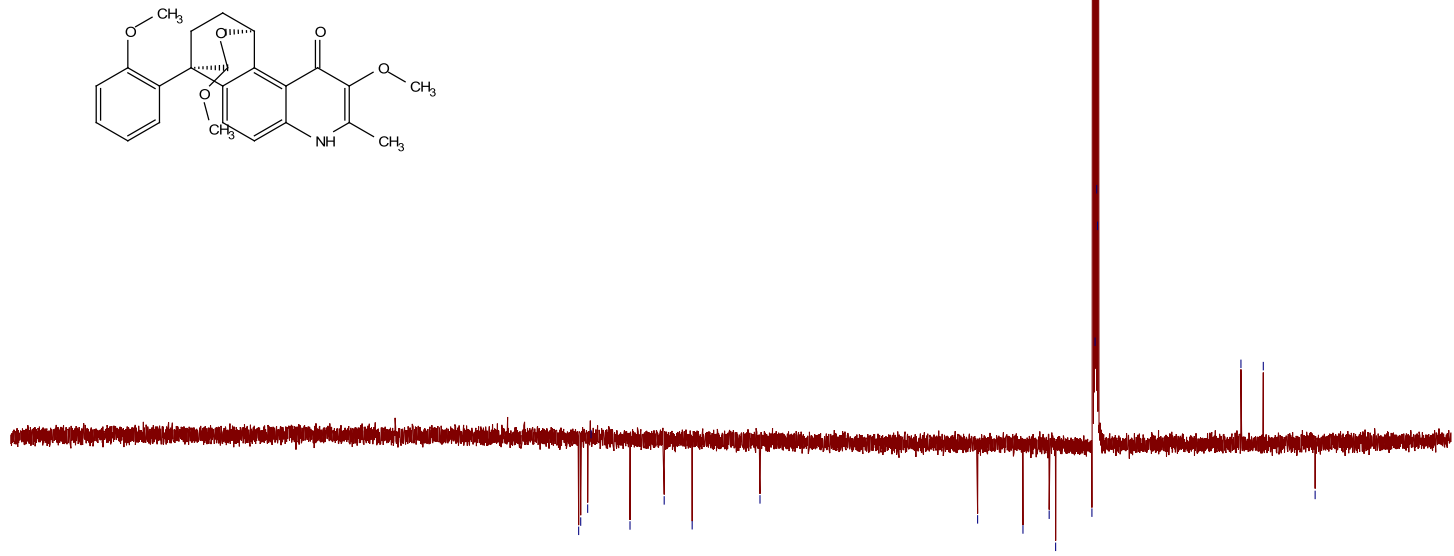

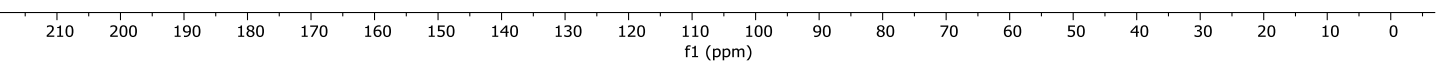

Figure S82. DEPTQ (150 MHz, methanol- $\left.d_{4}\right)$ spectrum of13-Methoxywaltherione V (12).

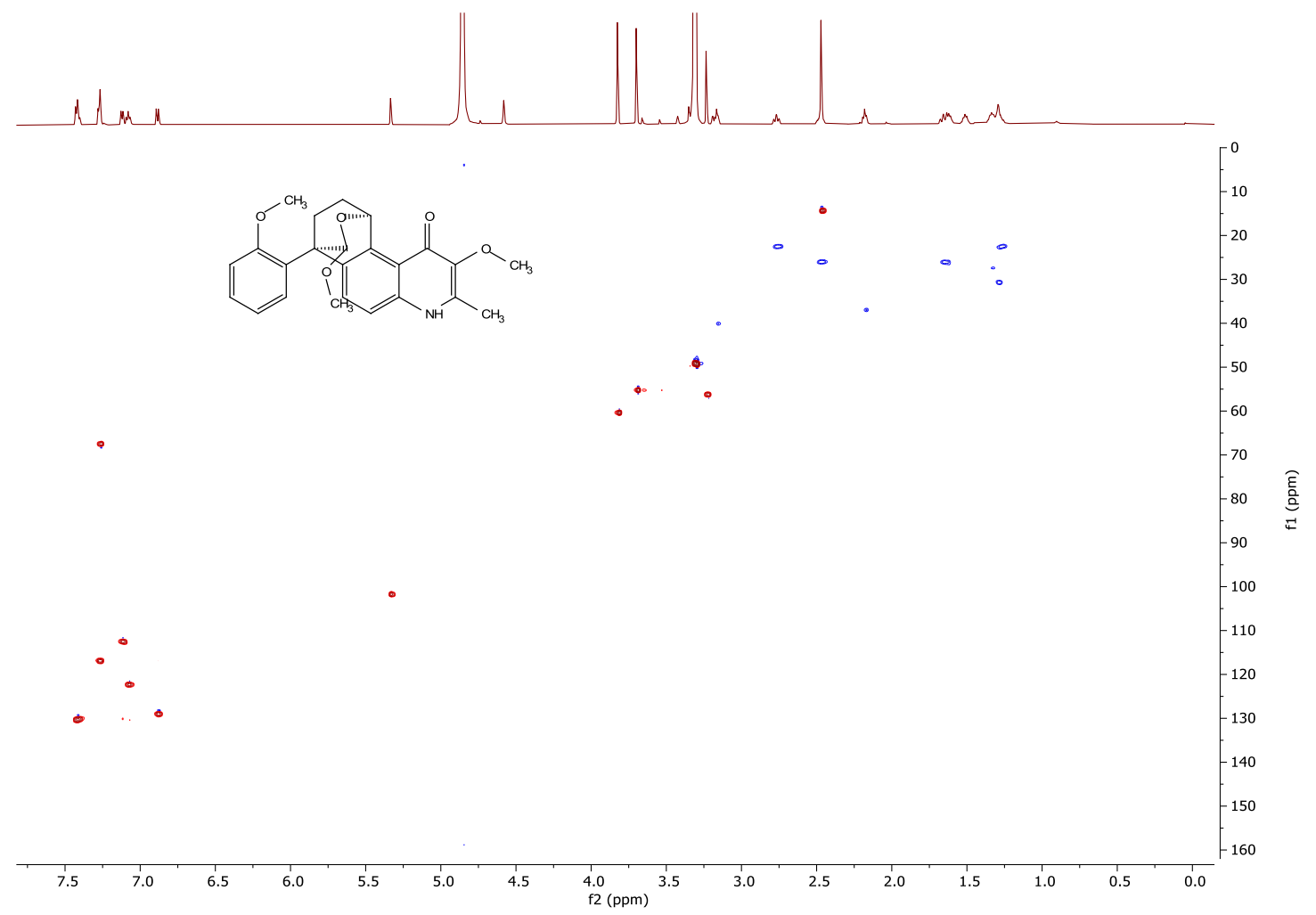

Figure S83. HSQC (600 MHz, methanol- $d_{4}$ ) spectrum of 13-Methoxywaltherione V (12). 


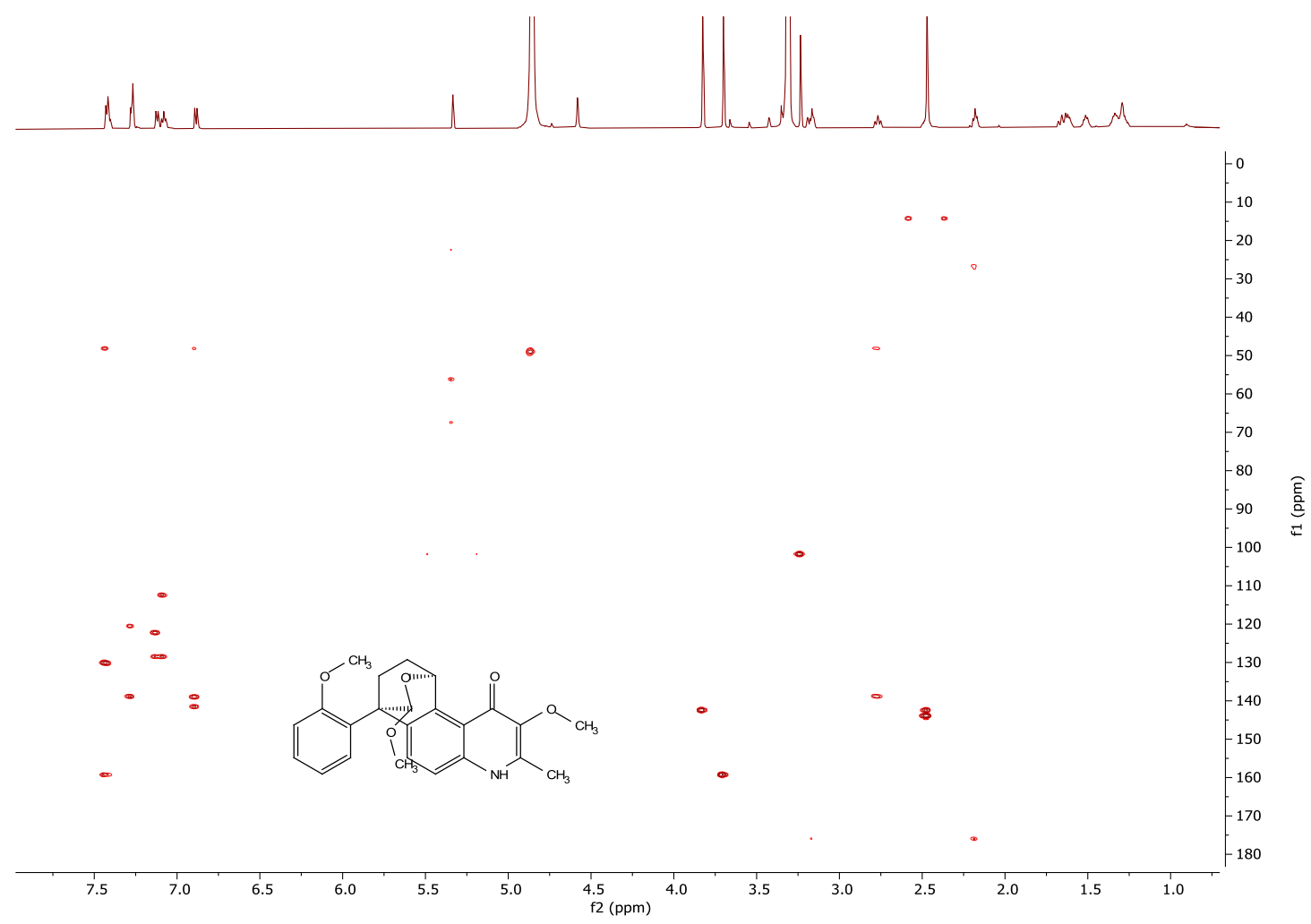

Figure S84. HMBC (600 MHz, methanol- $\left.d_{4}\right)$ spectrum of 13-Methoxywaltherione V (12).

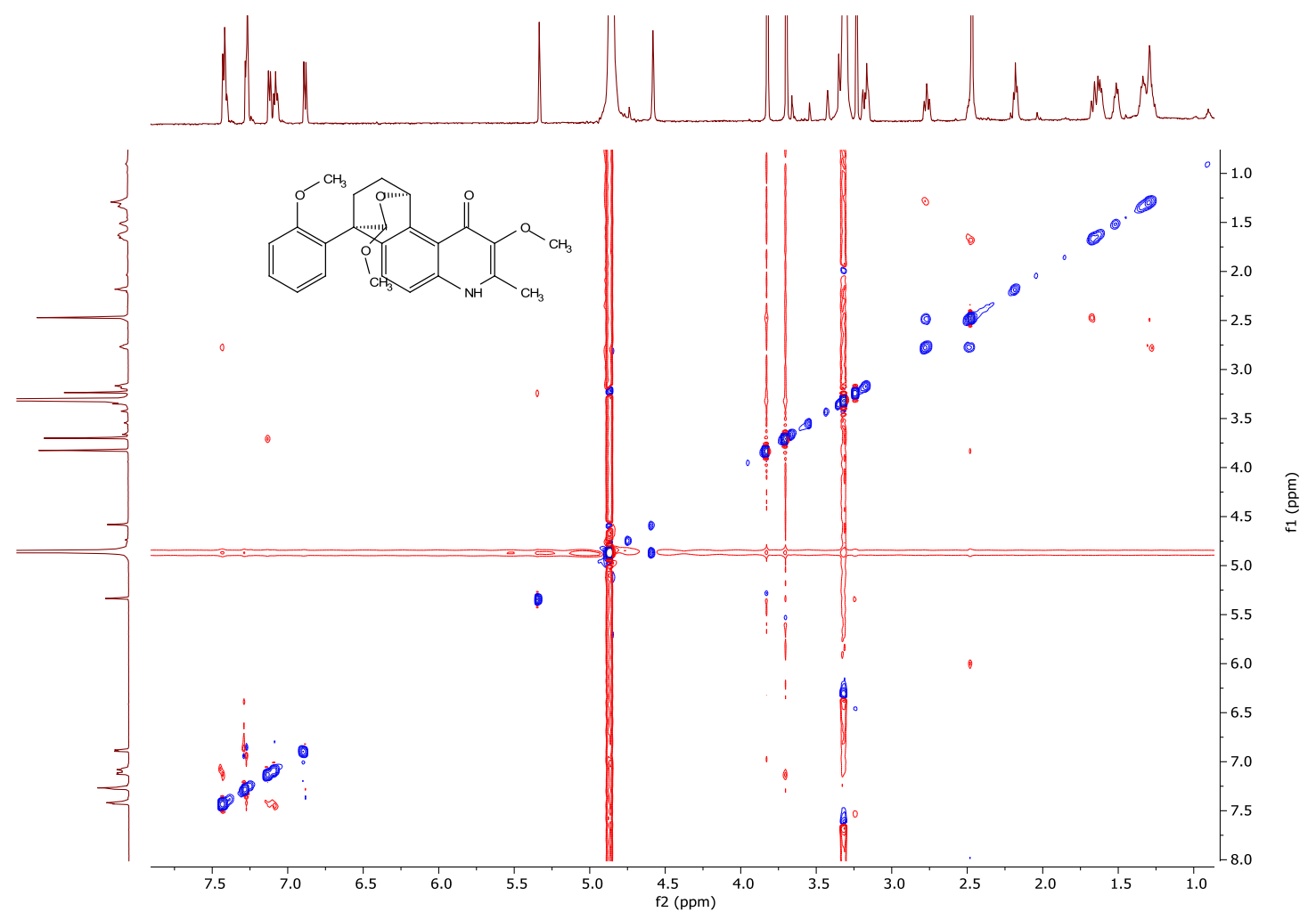

Figure S85. ROESY (600 MHz, methanol- $\left.d_{4}\right)$ spectrum of 13-Methoxywaltherione V (12). 
Figure S86. Calculation data for ECD curves of compounds 4, 8, 9, 11 and 12

Cartesian coordinates for the low-energy optimized conformer of 4 at cam-B3LYP/6-31G (d, p) level of theory in $\mathrm{MeOH}$

\begin{tabular}{|c|c|c|c|c|c|c|c|}
\hline Atom type & $X$ & $\mathrm{Y}$ & $\mathrm{Z}$ & Atom type & $X$ & $\mathrm{Y}$ & $\mathrm{Z}$ \\
\hline $\mathrm{C}$ & 2.352758 & 2.593346 & 1.698122 & $\mathrm{H}$ & 1.118209 & 1.181234 & -3.14067 \\
\hline $\mathrm{C}$ & 2.229675 & 1.367825 & 0.849868 & $\mathrm{H}$ & -0.96665 & -0.90656 & -2.40929 \\
\hline $\mathrm{C}$ & 2.706361 & 0.143432 & 1.217515 & $\mathrm{H}$ & -0.48589 & -0.36172 & -4.0047 \\
\hline $\mathrm{O}$ & 3.414479 & 0.039825 & 2.386623 & $\mathrm{H}$ & 0.531164 & -2.58286 & -3.54764 \\
\hline $\mathrm{C}$ & 2.823445 & -0.81725 & 3.363512 & $\mathrm{H}$ & 1.766393 & -1.34481 & -3.70225 \\
\hline $\mathrm{C}$ & 2.556203 & -1.02709 & 0.37211 & $\mathrm{H}$ & 2.499447 & -2.48916 & -1.83497 \\
\hline $\mathrm{C}$ & 1.733783 & -0.84417 & -0.81301 & $\mathrm{H}$ & 1.226087 & -3.40861 & -0.03666 \\
\hline $\mathrm{C}$ & 1.29212 & 0.401452 & -1.16579 & $\mathrm{H}$ & 0.876711 & -4.1016 & -1.60649 \\
\hline $\mathrm{N}$ & 1.596191 & 1.470723 & -0.36157 & $\mathrm{H}$ & -1.13376 & -4.05428 & -0.37884 \\
\hline $\mathrm{O}$ & 1.136304 & 2.721489 & -0.72672 & $\mathrm{H}$ & -1.31524 & -2.90976 & -1.68221 \\
\hline $\mathrm{C}$ & 2.104493 & 3.432427 & -1.51507 & $\mathrm{H}$ & -0.97496 & -1.01931 & -0.10789 \\
\hline $\mathrm{C}$ & 0.478141 & 0.687854 & -2.39979 & $\mathrm{H}$ & -0.59945 & -2.12891 & 1.190397 \\
\hline $\mathrm{C}$ & -0.10751 & -0.58494 & -3.00314 & $\mathrm{H}$ & -2.87523 & -3.06176 & 1.131711 \\
\hline $\mathrm{C}$ & 0.943088 & -1.69043 & -3.06547 & $\mathrm{H}$ & -3.29481 & -2.08681 & -0.27367 \\
\hline $\mathrm{C}$ & 1.499607 & -2.06419 & -1.68422 & $\mathrm{H}$ & -2.50581 & -0.9319 & 2.434457 \\
\hline $\mathrm{C}$ & 0.723698 & -3.20967 & -0.98656 & $\mathrm{H}$ & -4.54211 & -2.13654 & 2.320352 \\
\hline $\mathrm{C}$ & -0.78265 & -3.07833 & -0.73838 & $\mathrm{H}$ & -1.26745 & 0.970914 & 1.691535 \\
\hline $\mathrm{C}$ & -1.20025 & -2.01619 & 0.278515 & $\mathrm{H}$ & -1.19769 & 3.114029 & 0.467054 \\
\hline $\mathrm{C}$ & -2.68186 & -2.10085 & 0.635764 & $\mathrm{H}$ & -3.07988 & 3.76073 & -1.01707 \\
\hline $\mathrm{C}$ & -3.17309 & -0.97982 & 1.560434 & $\mathrm{H}$ & -5.01296 & 2.228956 & -1.28585 \\
\hline $\mathrm{O}$ & -4.50934 & -1.23267 & 1.9829 & $\mathrm{H}$ & -5.05838 & 0.067678 & -0.07635 \\
\hline $\mathrm{C}$ & -3.16158 & 0.36841 & 0.875038 & & & & \\
\hline $\mathrm{C}$ & -2.08665 & 1.24176 & 1.030983 & & & & \\
\hline $\mathrm{C}$ & -2.05108 & 2.455062 & 0.349074 & & & & \\
\hline $\mathrm{C}$ & -3.10217 & 2.813363 & -0.48805 & & & & \\
\hline $\mathrm{C}$ & -4.18613 & 1.952764 & -0.63894 & & & & \\
\hline $\mathrm{C}$ & -4.21319 & 0.737925 & 0.034957 & & & & \\
\hline $\mathrm{O}$ & 3.088944 & -2.11613 & 0.66417 & & & & \\
\hline $\mathrm{H}$ & 3.017782 & 3.335595 & 1.250011 & & & & \\
\hline $\mathrm{H}$ & 1.378598 & 3.065169 & 1.839465 & & & & \\
\hline $\mathrm{H}$ & 2.764194 & 2.306421 & 2.663121 & & & & \\
\hline $\mathrm{H}$ & 3.491314 & -0.81004 & 4.225603 & & & & \\
\hline $\mathrm{H}$ & 2.727557 & -1.8325 & 2.975643 & & & & \\
\hline $\mathrm{H}$ & 1.84061 & -0.43873 & 3.665766 & & & & \\
\hline $\mathrm{H}$ & 1.64562 & 4.400885 & -1.71024 & & & & \\
\hline $\mathrm{H}$ & 2.299358 & 2.915855 & -2.45661 & & & & \\
\hline $\mathrm{H}$ & 3.03733 & 3.565646 & -0.96389 & & & & \\
\hline $\mathrm{H}$ & -0.30992 & 1.401451 & -2.14951 & & & & \\
\hline
\end{tabular}


Cartesian coordinates for the low-energy optimized conformer of 8 at cam-B3LYP/6-31G (d, p) level of theory in $\mathrm{MeOH}$

\begin{tabular}{|c|c|c|c|c|c|c|c|}
\hline Atom type & $X$ & $\mathrm{Y}$ & $\mathrm{Z}$ & Atom type & $X$ & $\mathrm{Y}$ & $\mathrm{Z}$ \\
\hline $\mathrm{C}$ & 5.13241 & 2.023579 & -0.19756 & $\mathrm{H}$ & 0.346665 & 3.167855 & 0.907814 \\
\hline $\mathrm{O}$ & 4.742836 & 0.88731 & -0.9695 & $\mathrm{H}$ & -1.58016 & 2.993919 & 2.659612 \\
\hline $\mathrm{C}$ & 3.620003 & 0.243633 & -0.50861 & $\mathrm{H}$ & -0.62799 & 1.54899 & 2.397873 \\
\hline $\mathrm{C}$ & 3.75516 & -1.03787 & -0.06177 & $\mathrm{H}$ & -3.45046 & 2.124878 & 1.378375 \\
\hline $\mathrm{C}$ & 5.091174 & -1.74143 & 0.019807 & $\mathrm{H}$ & -2.90123 & 0.802205 & 2.4077 \\
\hline $\mathrm{O}$ & 4.937577 & -3.12494 & 0.273679 & $\mathrm{H}$ & -3.5168 & 0.444033 & -2.1952 \\
\hline $\mathrm{N}$ & 2.687635 & -1.75537 & 0.354265 & $\mathrm{H}$ & -5.50652 & -0.84216 & -2.9192 \\
\hline $\mathrm{C}$ & 1.409843 & -1.24864 & 0.363843 & $\mathrm{H}$ & -6.78252 & -2.18731 & -1.26935 \\
\hline $\mathrm{C}$ & 0.341042 & -2.03828 & 0.821297 & $\mathrm{H}$ & -6.05246 & -2.22856 & 1.102566 \\
\hline $\mathrm{C}$ & -0.94215 & -1.52849 & 0.830672 & $\mathrm{H}$ & -4.08952 & -0.93928 & 1.817264 \\
\hline $\mathrm{C}$ & -1.15941 & -0.23411 & 0.359018 & & & & \\
\hline $\mathrm{C}$ & -0.11527 & 0.541031 & -0.09992 & & & & \\
\hline $\mathrm{C}$ & -0.69612 & 1.883138 & -0.48871 & & & & \\
\hline $\mathrm{C}$ & -0.68009 & 2.864534 & 0.687347 & & & & \\
\hline $\mathrm{C}$ & -1.3421 & 2.225722 & 1.918612 & & & & \\
\hline $\mathrm{C}$ & -2.61745 & 1.439443 & 1.565812 & & & & \\
\hline $\mathrm{C}$ & -2.42865 & 0.610174 & 0.276667 & & & & \\
\hline $\mathrm{O}$ & -2.07104 & 1.55233 & -0.74716 & & & & \\
\hline $\mathrm{C}$ & -3.66421 & -0.15522 & -0.14709 & & & & \\
\hline $\mathrm{C}$ & -4.07801 & -0.14301 & -1.479 & & & & \\
\hline $\mathrm{C}$ & -5.19509 & -0.86732 & -1.87968 & & & & \\
\hline $\mathrm{C}$ & -5.91111 & -1.62153 & -0.95624 & & & & \\
\hline $\mathrm{C}$ & -5.50202 & -1.64403 & 0.372433 & & & & \\
\hline $\mathrm{C}$ & -4.38872 & -0.91382 & 0.774731 & & & & \\
\hline $\mathrm{C}$ & 1.204594 & 0.071245 & -0.09129 & & & & \\
\hline $\mathrm{C}$ & 2.330427 & 0.894716 & -0.56006 & & & & \\
\hline $\mathrm{O}$ & 2.180443 & 2.059949 & -0.96185 & & & & \\
\hline $\mathrm{H}$ & 6.040188 & 2.415091 & -0.65807 & & & & \\
\hline $\mathrm{H}$ & 4.349809 & 2.784171 & -0.21335 & & & & \\
\hline $\mathrm{H}$ & 5.347984 & 1.736193 & 0.837488 & & & & \\
\hline $\mathrm{H}$ & 5.660402 & -1.54505 & -0.89189 & & & & \\
\hline $\mathrm{H}$ & 5.660581 & -1.32194 & 0.854327 & & & & \\
\hline $\mathrm{H}$ & 4.828279 & -3.58105 & -0.57049 & & & & \\
\hline $\mathrm{H}$ & 2.880221 & -2.70462 & 0.653859 & & & & \\
\hline $\mathrm{H}$ & 0.537142 & -3.04901 & 1.163788 & & & & \\
\hline $\mathrm{H}$ & -1.76567 & -2.13803 & 1.18774 & & & & \\
\hline $\mathrm{H}$ & -0.26278 & 2.312155 & -1.38972 & & & & \\
\hline $\mathrm{H}$ & -1.23262 & 3.756965 & 0.375794 & & & & \\
\hline
\end{tabular}


Cartesian coordinates for the low-energy optimized conformer of 9 at cam-B3LYP/6-31G (d, p) level of theory in $\mathrm{MeOH}$

\begin{tabular}{|c|c|c|c|c|c|c|c|}
\hline Atom type & $X$ & $\mathrm{Y}$ & $\mathrm{Z}$ & Atom type & $X$ & $\mathrm{Y}$ & $\mathrm{Z}$ \\
\hline $\mathrm{C}$ & 5.332567 & 2.225527 & -0.11455 & $\mathrm{C}$ & 1.675905 & 1.516373 & -0.36697 \\
\hline $\mathrm{C}$ & 4.057062 & 1.453419 & -0.00056 & $\mathrm{~N}$ & 2.924181 & 2.091532 & -0.40911 \\
\hline $\mathrm{C}$ & 3.986402 & 0.182208 & 0.495653 & $\mathrm{O}$ & 2.656864 & -1.69063 & 1.042254 \\
\hline $\mathrm{O}$ & 5.130067 & -0.42013 & 0.95955 & $\mathrm{H}$ & 6.165973 & 1.619223 & 0.231963 \\
\hline $\mathrm{C}$ & 5.577633 & -1.51796 & 0.165477 & $\mathrm{H}$ & 5.283395 & 3.136443 & 0.489224 \\
\hline $\mathrm{C}$ & 2.730012 & -0.5334 & 0.601057 & $\mathrm{H}$ & 5.510975 & 2.521859 & -1.15211 \\
\hline $\mathrm{C}$ & 1.548846 & 0.208236 & 0.140442 & $\mathrm{H}$ & 4.815649 & -2.2985 & 0.121671 \\
\hline $\mathrm{C}$ & 0.257061 & -0.32921 & 0.202724 & $\mathrm{H}$ & 6.474899 & -1.90641 & 0.648873 \\
\hline $\mathrm{C}$ & -0.22588 & -1.68558 & 0.660942 & $\mathrm{H}$ & 5.828373 & -1.18747 & -0.84866 \\
\hline $\mathrm{C}$ & -0.18202 & -2.70834 & -0.47563 & $\mathrm{H}$ & 0.256913 & -2.05076 & 1.564612 \\
\hline $\mathrm{C}$ & -0.94197 & -2.16721 & -1.69446 & $\mathrm{H}$ & 0.854074 & -2.9406 & -0.734 \\
\hline $\mathrm{C}$ & -2.25156 & -1.46502 & -1.32627 & $\mathrm{H}$ & -0.64918 & -3.62852 & -0.11096 \\
\hline $\mathrm{O}$ & -2.68591 & -0.77477 & -2.48486 & $\mathrm{H}$ & -0.32269 & -1.44074 & -2.22976 \\
\hline $\mathrm{C}$ & -2.05761 & -0.53879 & -0.08361 & $\mathrm{H}$ & -1.16295 & -2.97033 & -2.40244 \\
\hline $\mathrm{O}$ & -1.61216 & -1.43143 & 0.946681 & $\mathrm{H}$ & -2.99618 & -2.20972 & -1.01708 \\
\hline $\mathrm{C}$ & -3.33895 & 0.13901 & 0.352914 & $\mathrm{H}$ & -3.63788 & -0.63584 & -2.41427 \\
\hline $\mathrm{C}$ & -3.98416 & 1.067561 & -0.4707 & $\mathrm{H}$ & -3.5651 & 1.320476 & -1.43736 \\
\hline $\mathrm{C}$ & -5.16636 & 1.676015 & -0.06269 & $\mathrm{H}$ & -5.65047 & 2.395045 & -0.71558 \\
\hline $\mathrm{C}$ & -5.72395 & 1.367229 & 1.172668 & $\mathrm{H}$ & -6.64608 & 1.842496 & 1.490669 \\
\hline $\mathrm{C}$ & -5.08864 & 0.445182 & 1.99697 & $\mathrm{H}$ & -5.51436 & 0.196823 & 2.964022 \\
\hline $\mathrm{C}$ & -3.90655 & -0.16406 & 1.590854 & $\mathrm{H}$ & -3.41001 & -0.87944 & 2.233869 \\
\hline $\mathrm{C}$ & -0.84362 & 0.368283 & -0.25247 & $\mathrm{H}$ & -1.55627 & 2.215911 & -1.13134 \\
\hline $\mathrm{C}$ & -0.70035 & 1.653793 & -0.7776 & $\mathrm{H}$ & 0.683821 & 3.233189 & -1.212 \\
\hline $\mathrm{C}$ & 0.55425 & 2.228208 & -0.82395 & $\mathrm{H}$ & 3.008043 & 3.032894 & -0.76614 \\
\hline
\end{tabular}


Cartesian coordinates for the low-energy optimized conformer of $\mathbf{1 1}$ at cam-B3LYP/6-31G (d, p) level of theory in $\mathrm{MeOH}$

\begin{tabular}{|c|c|c|c|c|c|c|c|}
\hline Atom type & $X$ & $\mathrm{Y}$ & $\mathrm{Z}$ & Atom type & $X$ & $\mathrm{Y}$ & $\mathrm{Z}$ \\
\hline $\mathrm{C}$ & 0.684444 & 0.057529 & -0.27739 & $\mathrm{H}$ & 0.645195 & 3.463585 & 1.578136 \\
\hline $\mathrm{C}$ & 0.687983 & -1.29904 & -0.62664 & $\mathrm{H}$ & 1.721419 & 1.681973 & -2.1808 \\
\hline $\mathrm{C}$ & -0.49643 & -1.9817 & -0.76798 & $\mathrm{H}$ & 2.49833 & 2.826741 & -1.06404 \\
\hline $\mathrm{C}$ & -1.71769 & -1.32141 & -0.55729 & $\mathrm{H}$ & 5.194353 & -2.206 & 1.070856 \\
\hline $\mathrm{C}$ & -1.75762 & 0.045654 & -0.21862 & $\mathrm{H}$ & 6.70827 & -1.51218 & -0.73397 \\
\hline $\mathrm{C}$ & -0.521 & 0.724494 & -0.10767 & $\mathrm{H}$ & 6.025654 & 0.303946 & -2.29625 \\
\hline $\mathrm{N}$ & -2.8903 & -2.02714 & -0.69515 & $\mathrm{H}$ & 3.858007 & 1.389507 & -2.02184 \\
\hline $\mathrm{C}$ & -4.12169 & -1.47706 & -0.51803 & $\mathrm{H}$ & 4.014345 & -1.98532 & 2.987703 \\
\hline $\mathrm{C}$ & -4.2264 & -0.15656 & -0.19159 & $\mathrm{H}$ & 2.269714 & -2.32835 & 3.082501 \\
\hline $\mathrm{C}$ & -3.06562 & 0.694434 & -0.00322 & $\mathrm{H}$ & 3.268523 & -3.14778 & 1.856108 \\
\hline $\mathrm{C}$ & -5.29602 & -2.38186 & -0.71268 & & & & \\
\hline $\mathrm{O}$ & -5.47433 & 0.40677 & -0.07592 & & & & \\
\hline $\mathrm{C}$ & -5.84764 & 0.738495 & 1.259999 & & & & \\
\hline $\mathrm{O}$ & -3.19895 & 1.884039 & 0.325348 & & & & \\
\hline $\mathrm{C}$ & -0.31427 & 2.199443 & 0.139517 & & & & \\
\hline $\mathrm{C}$ & 1.909268 & 0.950343 & -0.12687 & & & & \\
\hline $\mathrm{C}$ & 1.843477 & 1.657687 & 1.261528 & & & & \\
\hline $\mathrm{C}$ & 0.495397 & 2.392135 & 1.421114 & & & & \\
\hline $\mathrm{O}$ & 0.449272 & 2.731475 & -0.9581 & & & & \\
\hline $\mathrm{C}$ & 1.698167 & 2.082049 & -1.15995 & & & & \\
\hline $\mathrm{C}$ & 3.244423 & 0.241318 & -0.31496 & & & & \\
\hline $\mathrm{C}$ & 3.653891 & -0.79331 & 0.55606 & & & & \\
\hline $\mathrm{C}$ & 4.890926 & -1.41435 & 0.398654 & & & & \\
\hline $\mathrm{C}$ & 5.747187 & -1.02042 & -0.62493 & & & & \\
\hline $\mathrm{C}$ & 5.368848 & -0.01118 & -1.49298 & & & & \\
\hline $\mathrm{C}$ & 4.129297 & 0.603097 & -1.32869 & & & & \\
\hline $\mathrm{O}$ & 2.775948 & -1.14931 & 1.533849 & & & & \\
\hline $\mathrm{C}$ & 3.11552 & -2.21357 & 2.405913 & & & & \\
\hline $\mathrm{H}$ & 1.625283 & -1.81641 & -0.79214 & & & & \\
\hline $\mathrm{H}$ & -0.5001 & -3.03251 & -1.03993 & & & & \\
\hline $\mathrm{H}$ & -2.83174 & -3.00612 & -0.93738 & & & & \\
\hline $\mathrm{H}$ & -6.21984 & -1.82865 & -0.56108 & & & & \\
\hline $\mathrm{H}$ & -5.29564 & -2.79899 & -1.72397 & & & & \\
\hline $\mathrm{H}$ & -5.25878 & -3.21687 & -0.00724 & & & & \\
\hline $\mathrm{H}$ & -5.88374 & -0.15868 & 1.888256 & & & & \\
\hline $\mathrm{H}$ & -5.15038 & 1.461296 & 1.687989 & & & & \\
\hline $\mathrm{H}$ & -6.84464 & 1.177819 & 1.205121 & & & & \\
\hline $\mathrm{H}$ & -1.25025 & 2.745469 & 0.15155 & & & & \\
\hline $\mathrm{H}$ & 1.989924 & 0.920088 & 2.047328 & & & & \\
\hline $\mathrm{H}$ & 2.677535 & 2.36378 & 1.312308 & & & & \\
\hline $\mathrm{H}$ & -0.07173 & 2.012603 & 2.275566 & & & & \\
\hline
\end{tabular}


Cartesian coordinates for the low-energy optimized conformer of 12 at cam-B3LYP/6-31G $(d, p)$ level of theory in $\mathrm{MeOH}$

\begin{tabular}{|c|c|c|c|}
\hline Atom type & $X$ & $\mathrm{Y}$ & $\mathrm{Z}$ \\
\hline $\mathrm{C}$ & 5.439734 & -2.23426 & 1.186769 \\
\hline $\mathrm{C}$ & 4.251535 & -1.46573 & 0.704086 \\
\hline $\mathrm{C}$ & 4.333363 & -0.37357 & -0.10967 \\
\hline $\mathrm{O}$ & 5.566341 & 0.0287 & -0.5627 \\
\hline $\mathrm{C}$ & 5.994017 & 1.29232 & -0.05849 \\
\hline $\mathrm{C}$ & 3.157781 & 0.329862 & -0.58903 \\
\hline $\mathrm{C}$ & 1.865715 & -0.17024 & -0.08283 \\
\hline $\mathrm{C}$ & 0.619968 & 0.419903 & -0.40288 \\
\hline $\mathrm{C}$ & 0.398078 & 1.678984 & -1.20267 \\
\hline $\mathrm{C}$ & -0.43971 & 1.336794 & -2.43332 \\
\hline $\mathrm{C}$ & -1.80518 & 0.825144 & -1.93546 \\
\hline $\mathrm{C}$ & -1.80603 & 0.65381 & -0.38047 \\
\hline $\mathrm{C}$ & -1.5455 & 2.079806 & 0.160343 \\
\hline $\mathrm{O}$ & -1.41466 & 2.032577 & 1.554209 \\
\hline $\mathrm{C}$ & -1.26978 & 3.310791 & 2.148099 \\
\hline $\mathrm{O}$ & -0.38031 & 2.616167 & -0.42525 \\
\hline $\mathrm{C}$ & -3.13885 & 0.066765 & 0.074015 \\
\hline $\mathrm{C}$ & -4.02582 & 0.772956 & 0.884264 \\
\hline $\mathrm{C}$ & -5.27068 & 0.264852 & 1.246526 \\
\hline $\mathrm{C}$ & -5.65257 & -0.98812 & 0.798564 \\
\hline $\mathrm{C}$ & -4.79272 & -1.72923 & -0.00505 \\
\hline $\mathrm{C}$ & -3.55086 & -1.21105 & -0.36696 \\
\hline $\mathrm{O}$ & -2.67189 & -1.90015 & -1.14669 \\
\hline $\mathrm{C}$ & -3.01289 & -3.20734 & -1.57514 \\
\hline $\mathrm{C}$ & -0.57209 & -0.13148 & 0.040081 \\
\hline $\mathrm{C}$ & -0.55527 & -1.25922 & 0.869985 \\
\hline $\mathrm{C}$ & 0.638066 & -1.84385 & 1.218863 \\
\hline $\mathrm{C}$ & 1.847533 & -1.31212 & 0.741677 \\
\hline $\mathrm{N}$ & 3.029957 & -1.91882 & 1.095531 \\
\hline $\mathrm{O}$ & 3.265196 & 1.280967 & -1.37974 \\
\hline $\mathrm{H}$ & 5.460396 & -2.26404 & 2.279811 \\
\hline $\mathrm{H}$ & 6.353762 & -1.76965 & 0.824782 \\
\hline $\mathrm{H}$ & 5.401485 & -3.26614 & 0.825243 \\
\hline $\mathrm{H}$ & 6.974389 & 1.486408 & -0.49558 \\
\hline $\mathrm{H}$ & 6.085162 & 1.265002 & 1.033149 \\
\hline $\mathrm{H}$ & 5.29827 & 2.081019 & -0.3514 \\
\hline $\mathrm{H}$ & 1.327297 & 2.180514 & -1.44506 \\
\hline $\mathrm{H}$ & 0.078572 & 0.588855 & -3.03924 \\
\hline $\mathrm{H}$ & -0.55603 & 2.23507 & -3.04473 \\
\hline $\mathrm{H}$ & -2.59839 & 1.535558 & -2.1864 \\
\hline $\mathrm{H}$ & -2.06326 & -0.12639 & -2.39313 \\
\hline $\mathrm{H}$ & -2.37207 & 2.755854 & -0.10684 \\
\hline $\mathrm{H}$ & -1.22588 & 3.159634 & 3.227203 \\
\hline $\mathrm{H}$ & -2.12553 & 3.957688 & 1.912335 \\
\hline $\mathrm{H}$ & -0.35298 & 3.803576 & 1.812216 \\
\hline $\mathrm{H}$ & -3.7338 & 1.741037 & 1.266978 \\
\hline $\mathrm{H}$ & -5.92857 & 0.850436 & 1.879376 \\
\hline
\end{tabular}




\begin{tabular}{lrrr}
\hline Atom type & $\mathrm{X}$ & $\mathrm{Y}$ & $\mathrm{Z}$ \\
\hline $\mathrm{H}$ & -6.61754 & -1.40291 & 1.071095 \\
$\mathrm{H}$ & -5.09644 & -2.70922 & -0.34832 \\
$\mathrm{H}$ & -3.1736 & -3.87773 & -0.72477 \\
$\mathrm{H}$ & -3.90746 & -3.20318 & -2.2061 \\
$\mathrm{H}$ & -2.16426 & -3.5626 & -2.15824 \\
$\mathrm{H}$ & -1.4839 & -1.67159 & 1.245696 \\
$\mathrm{H}$ & 0.658573 & -2.7182 & 1.861641 \\
$\mathrm{H}$ & 2.987449 & -2.73809 & 1.685001
\end{tabular}

